\title{
A Local Desymmetrization Approach to Piperidinyl Acetic Acid $\gamma$-Secretase Modulators
}

Peng Cheng, Haigen $\mathrm{Lu}$ and Liansuo $\mathrm{Zu}^{*}$

School of Pharmaceutical Sciences, Key Laboratory of Bioorganic Phosphorus Chemistry \& Chemical Biology (Ministry of Education), Beijing Advanced Innovation Center for Structural Biology \& Frontier Research Center for Biological Structure, Tsinghua University, Beijing 100084, China

\section{Table of Contents}

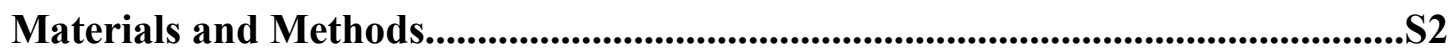

Experimental Procedures

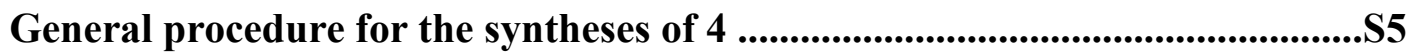

General procedure for the syntheses of 7 ......................................................57

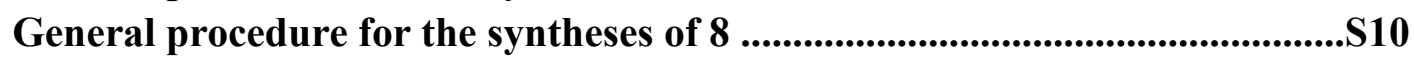

General procedure for the syntheses of 6 .................................................................S13

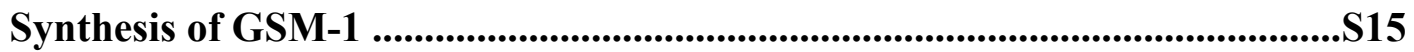

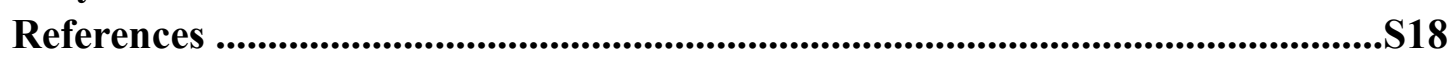

Crystallographic data for the opposite enantiomer of 6a...................................S18

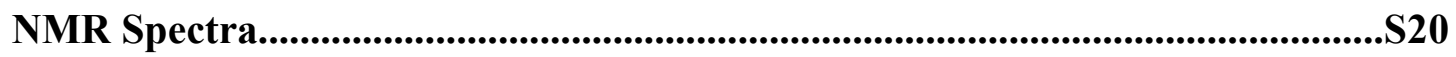




\section{Materials and methods.}

Unless stated otherwise, reactions were conducted in dry glassware using anhydrous solvents (passed through activated alumina columns). All commercially available reagents were used as received unless otherwise specified. Reaction temperatures were controlled using an IKA mag temperature modulator (oil bath), and unless stated otherwise, reactions were performed at room temperature (RT, approximately $23{ }^{\circ} \mathrm{C}$ ). Thin layer chromatography (TLC) was conducted on plates (GF254) supplied by Yantai Chemicals (China) and visualized using a combination of UV, anisaldehyde, iodine, and potassium permanganate staining. Silica gel (200-300 mesh) supplied by Tsingtao Haiyang Chemicals (China) was used for flash column chromatography. ${ }^{1} \mathrm{H}$ NMR spectra were recorded on Bruker spectrometers (at $400 \mathrm{MHz}$ ) and are reported relative to deuterated solvent signals. Data for ${ }^{1} \mathrm{H}$ NMR spectra are reported as follows: chemical shift $(\delta \mathrm{ppm})$, multiplicity, coupling constant $(\mathrm{Hz})$ and integration. ${ }^{13} \mathrm{C}$ NMR spectra are reported in terms of chemical shift. High resolution mass spectra (HRMS) were performed on Waters synapt G2 Q-TOF mass spectrometer (ESI). 


\section{General procedure for the syntheses of $4^{[1]}$}

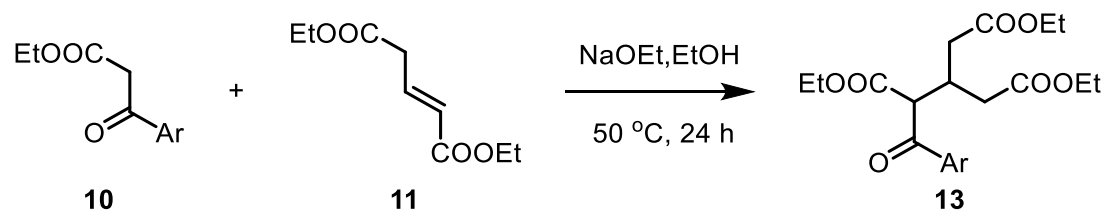

In a round-bottom flask equipped with a magnetic stirring bar, diethyl pent-2-enedioate 11 (610 mg, $3.28 \mathrm{mmol}, 1.0$ equiv) and $\mathbf{1 0}$ (3 equiv, $9.84 \mathrm{mmol}$ ) were dissolved in $\mathrm{EtOH}$ ( $6 \mathrm{~mL}$ ) under $\mathrm{N}_{2}$. A solution of $\mathrm{NaOEt}$ (446 mg, $6.56 \mathrm{mmol}, 2.0$ equiv) in $\mathrm{EtOH}$ ( $3 \mathrm{~mL}$ ) was added dropwise over $0.5 \mathrm{~h}$. The reaction was heated at $50{ }^{\circ} \mathrm{C}$ in an oil bath for 24 h. The reaction was quenched with aqueous $\mathrm{NH}_{4} \mathrm{Cl}$ and extracted with EtOAc $(30 \mathrm{~mL}$ $\mathrm{x} 2$ ). The organic layer was dried over $\mathrm{Na}_{2} \mathrm{SO}_{4}$ and concentrated in vacuo. The residue was purified by silica gel column chromatography to give the product.<smiles>CCOCC(CC(=O)OCC)C(COCC)C(=O)c1ccc(C(F)(F)F)cc1</smiles>

Purification by silica gel column chromatography (ethyl acetate/petroleum ether $=1: 10$ ) to yield the desired product $\left(1.23 \mathrm{~g}, 84 \%\right.$ yield) as colorless oil. ${ }^{1} \mathrm{H}$ NMR (400 MHz, Chloroform- $d$ ) $\delta 8.15(\mathrm{~d}, J=8.1 \mathrm{~Hz}, 2 \mathrm{H}), 7.75(\mathrm{~d}, J=8.0 \mathrm{~Hz}, 2 \mathrm{H}), 4.93(\mathrm{~d}, J=6.6 \mathrm{~Hz}$, $1 \mathrm{H}), 4.20-4.03(\mathrm{~m}, 6 \mathrm{H}), 3.26-3.09(\mathrm{~m}, 1 \mathrm{H}), 2.76-2.51(\mathrm{~m}, 4 \mathrm{H}), 1.25-1.16(\mathrm{~m}$, 9H). ${ }^{13} \mathrm{C}\left\{{ }^{1} \mathrm{H}\right\}$ NMR (100 MHz, Chloroform- $d$ ) $\delta$ 194.0, 172.1, 172.0, 168.3, 139.0, $134.9(\mathrm{q}, J=32.7 \mathrm{~Hz}), 129.0,125.9(\mathrm{q}, J=3.8 \mathrm{~Hz}), 123.5(\mathrm{q}, J=272.9 \mathrm{~Hz}), 61.7,60.6$, 60.6, 55.1, 35.6, 34.9, 31.45, 14.1, 13.9. $\left.{ }^{19} \mathrm{~F} \mathrm{NMR} \mathrm{(376} \mathrm{MHz,} \mathrm{CDCl}_{3}\right) \delta-63.3$. HRMS (ESI): calcd for $\mathrm{C}_{21} \mathrm{H}_{26} \mathrm{~F}_{3} \mathrm{O}_{7}[\mathrm{M}+\mathrm{H}]^{+}$: 447.1631, found 447.1644.<smiles>CCOCC(CC(=O)OCC)C(COCC)C(=O)c1ccccc1</smiles>

13b

Purification by silica gel column chromatography (ethyl acetate/petroleum ether $=1: 10$ ) to yield the desired product ( $881 \mathrm{mg}, 71 \%$ yield) as colorless oil. ${ }^{1} \mathrm{H}$ NMR (400 MHz, Chloroform- $d$ ) $\delta 7.99(\mathrm{dd}, J=8.3,1.3 \mathrm{~Hz}, 2 \mathrm{H}), 7.59-7.49(\mathrm{~m}, 1 \mathrm{H}), 7.42$ (dd, $J=8.3$, $7.0 \mathrm{~Hz}, 2 \mathrm{H}), 4.87(\mathrm{~d}, J=6.8 \mathrm{~Hz}, 1 \mathrm{H}), 4.18-3.96(\mathrm{~m}, 6 \mathrm{H}), 3.24-3.05(\mathrm{~m}, 1 \mathrm{H}), 2.67-$ $2.46(\mathrm{~m}, 4 \mathrm{H}), 1.22-1.02(\mathrm{~m}, 9 \mathrm{H}) .{ }^{13} \mathrm{C}\left\{{ }^{1} \mathrm{H}\right\}$ NMR $(100 \mathrm{MHz}$, Chloroform- $d) \delta 194.7$, 172.1, 172.0, 168.7, 136.2, 133.7, 128.8, 61.4, 60.5, 60.5, 54.9, 35.6, 34.9, 31.5, 14.1, 14.1, 13.9. HRMS (ESI): calcd for $\mathrm{C}_{20} \mathrm{H}_{27} \mathrm{O}_{7}[\mathrm{M}+\mathrm{H}]^{+}: 379.1757$, found 379.1775 . 
<smiles>CCOCC(COCC)C(COCC)C(=O)c1cccc(F)c1</smiles>

Purification by silica gel column chromatography (ethyl acetate/petroleum ether $=1: 10$ ) to yield the desired product ( $650 \mathrm{mg}, 50 \%$ yield) as colorless oil. ${ }^{1} \mathrm{H}$ NMR (400 MHz, Chloroform- $d$ ) $\delta 7.83(\mathrm{~d}, J=7.8 \mathrm{~Hz}, 1 \mathrm{H}), 7.71(\mathrm{dd}, J=9.6,2.2 \mathrm{~Hz}, 1 \mathrm{H}), 7.53-7.43$ (m, 1H), $7.35-7.22(\mathrm{~m}, 1 \mathrm{H}), 4.86(\mathrm{dd}, J=6.7,1.6 \mathrm{~Hz}, 1 \mathrm{H}), 4.28-3.94(\mathrm{~m}, 6 \mathrm{H}), 3.21$ - $3.11(\mathrm{~m}, 1 \mathrm{H}), 2.77-2.41(\mathrm{~m}, 4 \mathrm{H}), 1.45-0.98(\mathrm{~m}, 9 \mathrm{H}) .{ }^{13} \mathrm{C}\left\{{ }^{1} \mathrm{H}\right\}$ NMR $(100 \mathrm{MHz}$, Chloroform- $d$ ) $\delta 193.6,172.1,172.0,168.4,162.9(\mathrm{~d}, J=248.1 \mathrm{~Hz}), 138.4(\mathrm{~d}, J=6.4$ $\mathrm{Hz}), 130.5(\mathrm{~d}, J=7.7 \mathrm{~Hz}), 124.5(\mathrm{~d}, J=2.9 \mathrm{~Hz}), 120.8(\mathrm{~d}, J=21.4 \mathrm{~Hz}), 115.4(\mathrm{~d}, J=$ $22.6 \mathrm{~Hz}), 61.6,60.6,60.6,55.1,35.6,34.9,31.5,14.1,13.9 .{ }^{19} \mathrm{~F}$ NMR (376 MHz, Chloroform- $d$ ) $\delta$-111.4.; HRMS (ESI): calcd for $\mathrm{C}_{20} \mathrm{H}_{26} \mathrm{FO}_{7}[\mathrm{M}+\mathrm{H}]^{+}: 397.1663$, found 397.1665 .<smiles>CCOCC(COCC)C(COCC)C(=O)c1cccc(Cl)c1</smiles>

Purification by silica gel column chromatography (ethyl acetate/petroleum ether $=1: 10$ ) to yield the desired product $\left(677 \mathrm{mg}, 50 \%\right.$ yield) as colorless oil. ${ }^{1} \mathrm{H}$ NMR (400 MHz, Chloroform- $d$ ) $\delta 7.99(\mathrm{t}, J=1.9 \mathrm{~Hz}, 1 \mathrm{H}), 7.92(\mathrm{~d}, J=7.9 \mathrm{~Hz}, 1 \mathrm{H}), 7.56(\mathrm{~d}, J=8.0 \mathrm{~Hz}$, $1 \mathrm{H}), 7.43(\mathrm{t}, J=7.9 \mathrm{~Hz}, 1 \mathrm{H}), 4.86(\mathrm{~d}, J=6.6 \mathrm{~Hz}, 1 \mathrm{H}), 4.22-4.03(\mathrm{~m}, 6 \mathrm{H}), 3.25-3.06$ $(\mathrm{m}, 1 \mathrm{H}), 2.72-2.50(\mathrm{~m}, 4 \mathrm{H}), 1.33-1.08(\mathrm{~m}, 9 \mathrm{H}) .{ }^{13} \mathrm{C}\left\{{ }^{1} \mathrm{H}\right\}$ NMR $(100 \mathrm{MHz}$, Chloroform- $d$ ) $\delta$ 193.6, 172.1, 172.0, 168.4, 137.8, 135.2, 133.6, 130.1, 128.7, 126.8, 61.6, 60.6, 60.6, 55.0, 35.6, 34.9, 31.5, 14.1, 13.9. HRMS (ESI): calcd for $\mathrm{C}_{20} \mathrm{H}_{26} \mathrm{ClO}_{7}$ $[\mathrm{M}+\mathrm{H}]^{+}:$413.1367, found 413.1360 .<smiles>CCOCCC(COCC)C(OCC)C(=O)c1ccc(C)cc1</smiles>

Purification by silica gel column chromatography (ethyl acetate/petroleum ether $=1: 10$ ) to yield the desired product ( $978 \mathrm{mg}, 76 \%$ yield) as colorless oil. ${ }^{1} \mathrm{H}$ NMR (400 MHz, Chloroform- $d$ ) $\delta 7.92(\mathrm{~d}, J=7.8 \mathrm{~Hz}, 2 \mathrm{H}), 7.25(\mathrm{~d}, J=7.7 \mathrm{~Hz}, 2 \mathrm{H}), 4.86(\mathrm{~d}, J=6.9 \mathrm{~Hz}$, $1 \mathrm{H}), 4.09(\mathrm{dt}, J=14.2,6.9 \mathrm{~Hz}, 6 \mathrm{H}), 3.25-3.04(\mathrm{~m}, 1 \mathrm{H}), 2.71-2.51(\mathrm{~m}, 4 \mathrm{H}), 2.39$ (s, $3 \mathrm{H}), 1.31-1.07(\mathrm{~m}, 9 \mathrm{H}) .{ }^{13} \mathrm{C}\left\{{ }^{1} \mathrm{H}\right\}$ NMR $(100 \mathrm{MHz}$, Chloroform- $d) \delta$ 194.3, 172.1, 172.1, 168.8, 144.7, 133.8, 129.5, 128.9, 61.4, 60.5, 60.5, 54.8, 35.6, 34.9, 31.6, 21.7, 14.1, 14.1, 14.0. HRMS (ESI): calcd for $\mathrm{C}_{21} \mathrm{H}_{29} \mathrm{O}_{7}[\mathrm{M}+\mathrm{H}]^{+}: 393.1907$, found 393.1913. 
<smiles>CCOCC(CC(=O)OCC)C(COCC)C(=O)c1ccncc1</smiles>

$13 f$

Purification by silica gel column chromatography (ethyl acetate/petroleum ether $=$ 1:2)to yield the desired product (622 mg, 50\% yield) as colorless oil; ${ }^{1} \mathrm{H}$ NMR (400 MHz, Chloroform- $d$ ) $\delta 8.84(\mathrm{~d}, J=5.5 \mathrm{~Hz}, 2 \mathrm{H}), 7.82(\mathrm{~d}, J=5.5 \mathrm{~Hz}, 2 \mathrm{H}), 4.88(\mathrm{~d}, J=$ $6.0 \mathrm{~Hz}, 1 \mathrm{H}), 4.35-3.94(\mathrm{~m}, 6 \mathrm{H}), 3.30-3.06(\mathrm{~m}, 1 \mathrm{H}), 2.93-2.29(\mathrm{~m}, 4 \mathrm{H}), 1.52-0.90$ $(\mathrm{m}, 9 \mathrm{H}) .{ }^{13} \mathrm{C}\left\{{ }^{1} \mathrm{H}\right\}$ NMR (100 MHz, Chloroform- $d$ ) $\delta$ 194.5, 172.1, 171.9, 168.0, 151.0, 142.4, 121.5, 61.8, 60.7, 60.7, 55.1, 35.5, 34.9, 31.3, 14.2, 13.9. HRMS (ESI): calcd for $\mathrm{C}_{19} \mathrm{H}_{26} \mathrm{NO}_{7}[\mathrm{M}+\mathrm{H}]^{+}: 380.1709$, found 380.1717 .

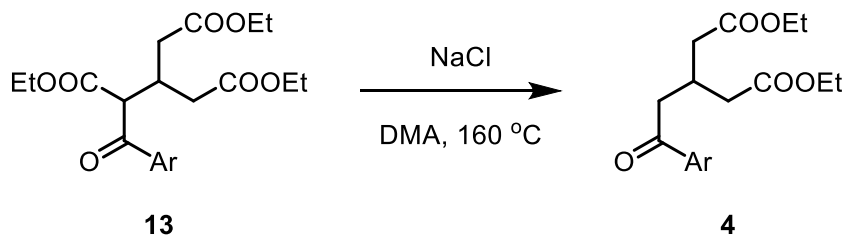

To the solution of $\mathbf{1 3}$ (1.66 mmol, 1.0 equiv) in dimethyl acetamide ( $9 \mathrm{~mL}$ ) was added $\mathrm{NaCl}$ (481 mg, $8.30 \mathrm{mmol}, 5.0$ equiv) at room temperature. The reaction mixture was stirred at $160{ }^{\circ} \mathrm{C}$ in an oil bath for $16 \mathrm{~h} . \mathrm{H}_{2} \mathrm{O}(45 \mathrm{~mL})$ was added to the resulting reaction mixture and the solution was extracted with EtOAc $(45 \mathrm{~mL})$. The organic layer was washed with water, dried over $\mathrm{Na}_{2} \mathrm{SO}_{4}$ and concentrated under reduced pressure. The residue was purified by silica gel column chromatography to give the product.<smiles>CCOCCC(COCC)CC(=O)c1ccc(C(F)(F)F)cc1</smiles>

Purification by silica gel column chromatography (ethyl acetate/petroleum ether $=1: 10$ ) to yield the desired product (559 mg, $90 \%$ yield) as colorless oil. ${ }^{1} \mathrm{H}$ NMR (400 MHz, Chloroform- $d$ ) $\delta 8.08(\mathrm{~d}, J=8.1 \mathrm{~Hz}, 2 \mathrm{H}), 7.72(\mathrm{~d}, J=8.1 \mathrm{~Hz}, 2 \mathrm{H}), 4.11(\mathrm{q}, J=7.2 \mathrm{~Hz}$, $4 \mathrm{H}), 3.18(\mathrm{~d}, J=6.6 \mathrm{~Hz}, 2 \mathrm{H}), 3.09-2.89(\mathrm{~m}, 1 \mathrm{H}), 2.51(\mathrm{~d}, J=6.3 \mathrm{~Hz}, 4 \mathrm{H}), 1.22(\mathrm{t}, J$ $=7.1 \mathrm{~Hz}, 6 \mathrm{H}) .{ }^{13} \mathrm{C}\left\{{ }^{1} \mathrm{H}\right\}$ NMR $(100 \mathrm{MHz}$, Chloroform- $d$ ) $\delta 197.8,172.1,139.5,134.4$ $(\mathrm{q}, J=32.4 \mathrm{~Hz}), 128.5,125.7(\mathrm{q}, J=3.7 \mathrm{~Hz}), 123.6(\mathrm{q}, J=272.3 \mathrm{~Hz}), 60.5,42.1,37.8$, 28.1, 14.2. ${ }^{19} \mathrm{~F}$ NMR (376 MHz, Chloroform- $d$ ) $\delta$-63.2. HRMS (ESI): calcd for $\mathrm{C}_{18} \mathrm{H}_{22} \mathrm{~F}_{3} \mathrm{O}_{5}[\mathrm{M}+\mathrm{H}]^{+}:$375.1419, found 375.1416.<smiles>CCOCC(CC(=O)OCC)CC(=O)c1ccccc1</smiles> 
Purification by silica gel column chromatography (ethyl acetate/petroleum ether $=1: 10$ ) to yield the desired product (432 mg, $85 \%$ yield) as colorless oil. ${ }^{1} \mathrm{H} \mathrm{NMR} \mathrm{(400} \mathrm{MHz,}$ Chloroform- $d$ ) $\delta 7.99-7.92(\mathrm{~m}, 2 \mathrm{H}), 7.58-7.48(\mathrm{~m}, 1 \mathrm{H}), 7.43(\mathrm{dd}, J=8.3,6.9 \mathrm{~Hz}$, 2H), $4.08(\mathrm{q}, J=7.1 \mathrm{~Hz}, 4 \mathrm{H}), 3.13(\mathrm{~d}, J=6.6 \mathrm{~Hz}, 2 \mathrm{H}), 2.96(\mathrm{~m}, J=6.6 \mathrm{~Hz}, 1 \mathrm{H}), 2.49$ $(\mathrm{d}, J=6.5 \mathrm{~Hz}, 4 \mathrm{H}), 1.20(\mathrm{t}, J=7.1 \mathrm{~Hz}, 6 \mathrm{H}) .{ }^{13} \mathrm{C}\left\{{ }^{1} \mathrm{H}\right\}$ NMR $(100 \mathrm{MHz}$, Chloroform- $d$ ) $\delta 198.7,172.1,136.8,133.2,128.6,128.1,60.4,41.7,37.9,28.2$, 14.2. HRMS (ESI): calcd for $\mathrm{C}_{17} \mathrm{H}_{23} \mathrm{O}_{5}[\mathrm{M}+\mathrm{H}]^{+}:$307.1545, found 307.1539 .

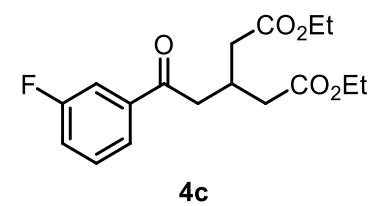

Purification by silica gel column chromatography (ethyl acetate/petroleum ether $=1: 10$ ) to yield the desired product (501 mg, $93 \%$ yield) as colorless oil. Colorless oil; ${ }^{1} \mathrm{H}$ NMR (400 MHz, Chloroform-d) $\delta 7.74$ (dd, $J=7.8,1.2 \mathrm{~Hz}, 1 \mathrm{H}$ ), 7.62 (ddd, $J=9.5$, 2.6, $1.5 \mathrm{~Hz}, 1 \mathrm{H}), 7.41(\mathrm{td}, J=7.9,5.5 \mathrm{~Hz}, 1 \mathrm{H}), 7.23(\mathrm{td}, J=8.2,2.5 \mathrm{~Hz}, 1 \mathrm{H}), 4.09$ (q, $J=7.1 \mathrm{~Hz}, 4 \mathrm{H}), 3.11(\mathrm{~d}, J=6.5 \mathrm{~Hz}, 2 \mathrm{H}), 3.03-2.87(\mathrm{~m}, 1 \mathrm{H}), 2.48(\mathrm{~d}, J=6.5 \mathrm{~Hz}, 4 \mathrm{H})$, $1.20(\mathrm{t}, J=7.1 \mathrm{~Hz}, 6 \mathrm{H}) .{ }^{13} \mathrm{C}\left\{{ }^{1} \mathrm{H}\right\} \mathrm{NMR}(100 \mathrm{MHz}$, Chloroform- $d) \delta 197.4(\mathrm{~d}, J=2.6$ $\mathrm{Hz}), 172.1,162.8$ (d, $J=247.9 \mathrm{~Hz}), 138.9$ (d, $J=6.3 \mathrm{~Hz}), 130.3$ (d, $J=7.5 \mathrm{~Hz}), 123.9$ $(\mathrm{d}, J=3.2 \mathrm{~Hz}), 120.2$ (d, $J=21.7 \mathrm{~Hz}), 114.8$ (d, $J=22.6 \mathrm{~Hz}), 60.5,41.9,37.81,28.1$, 14.2. ${ }^{19} \mathrm{~F}$ NMR (376 MHz, Chloroform- $d$ ) $\delta$-111.8. HRMS (ESI): calcd for $\mathrm{C}_{17} \mathrm{H}_{22} \mathrm{FO}_{5}$ $[\mathrm{M}+\mathrm{H}]^{+}: 325.1451$, found 325.1437 .<smiles>CCOCC(COCC)CC(=O)c1cccc(Cl)c1</smiles>

$4 d$

Purification by silica gel column chromatography (ethyl acetate/petroleum ether $=1: 10$ ) to yield the desired product $\left(520 \mathrm{mg}, 92 \%\right.$ yield) as colorless oil. ${ }^{1} \mathrm{H}$ NMR $(400 \mathrm{MHz}$, Chloroform- $d$ ) $\delta 7.91$ (t, $J=1.9 \mathrm{~Hz}, 1 \mathrm{H}), 7.83$ (dt, $J=7.7,1.3 \mathrm{~Hz}, 1 \mathrm{H}), 7.50$ (ddd, $J=$ $7.9,2.3,1.1 \mathrm{~Hz}, 1 \mathrm{H}), 7.38(\mathrm{t}, J=7.9 \mathrm{~Hz}, 1 \mathrm{H}), 4.09(\mathrm{q}, J=7.1 \mathrm{~Hz}, 4 \mathrm{H}), 3.11(\mathrm{~d}, J=6.5$ $\mathrm{Hz}, 2 \mathrm{H}), 3.02-2.87(\mathrm{~m}, 1 \mathrm{H}), 2.48(\mathrm{~d}, J=6.6 \mathrm{~Hz}, 4 \mathrm{H}), 1.21(\mathrm{t}, J=7.0 \mathrm{~Hz}, 6 \mathrm{H}) .{ }^{13} \mathrm{C}\left\{{ }^{1} \mathrm{H}\right\}$ NMR (100 MHz, Chloroform-d) $\delta$ 197.4, 172.1, 138.3, 134.9, 133.1, 130.0, 128.2, 126.2, 60.5, 41.8, 37.8, 28.1, 14.2. HRMS (ESI): calcd for $\mathrm{C}_{17} \mathrm{H}_{22} \mathrm{ClO}_{5}[\mathrm{M}+\mathrm{H}]^{+}$: 341.1156 , found 341.1154 .

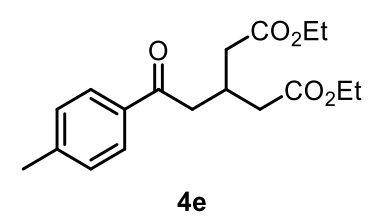

Purification by silica gel column chromatography (ethyl acetate/petroleum ether $=1: 10$ ) to yield the desired product (463 mg, $87 \%$ yield) as colorless oil. ${ }^{1} \mathrm{H}$ NMR (400 MHz, Chloroform- $d$ ) $\delta 7.87(\mathrm{~d}, J=8.1 \mathrm{~Hz}, 2 \mathrm{H}), 7.25(\mathrm{~d}, J=8.1 \mathrm{~Hz}, 2 \mathrm{H}), 4.11(\mathrm{q}, J=7.1,4 \mathrm{H})$, $3.12(\mathrm{~d}, J=6.7,2 \mathrm{H}), 3.05-2.89(\mathrm{~m}, 1 \mathrm{H}), 2.51(\mathrm{~d}, J=6.6,4 \mathrm{H}), 2.40(\mathrm{~s}, 3 \mathrm{H}), 1.23(\mathrm{t}, J$ 
$=7.2,6 \mathrm{H}) .{ }^{13} \mathrm{C}\left\{{ }^{1} \mathrm{H}\right\}$ NMR $(100 \mathrm{MHz}$, Chloroform- $d) \delta 198.3,172.2,144.0,134.4$, 129.3, 128.2, 60.4, 41.7, 38.0, 28.3, 21.6, 14.2. HRMS (ESI): calcd for $\mathrm{C}_{18} \mathrm{H}_{24} \mathrm{O}_{5}$ $[\mathrm{M}+\mathrm{H}]^{+}:$321.1702, found 321.1702.

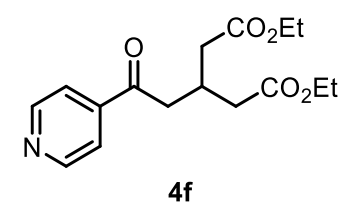

Purification by silica gel column chromatography (ethyl acetate/petroleum ether $=1: 1$ ) to yield the desired product (454 mg, $89 \%$ yield) as colorless oil. ${ }^{1} \mathrm{H}$ NMR (400 MHz, Chloroform- $d$ ) $\delta 8.84-8.70(\mathrm{~d}, J=4.8 \mathrm{~Hz} 2 \mathrm{H}), 7.80-7.63(\mathrm{~d}, J=4.8 \mathrm{~Hz}, 2 \mathrm{H}), 4.10$ (q, $J=7.1 \mathrm{~Hz}, 4 \mathrm{H}), 3.15(\mathrm{~d}, J=6.5 \mathrm{~Hz}, 2 \mathrm{H}), 3.02-2.87(\mathrm{~m}, 1 \mathrm{H}), 2.49$ (dd, $J=6.5,1.9$ $\mathrm{Hz}, 4 \mathrm{H}), 1.21(\mathrm{t}, J=7.1 \mathrm{~Hz}, 6 \mathrm{H}) .{ }^{13} \mathrm{C}$ NMR $(100 \mathrm{MHz}$, Chloroform-d) $\delta$ 198.3, 172.0, 151.0, 142.5, 121.1, 60.6, 42.0, 37.7, 27.9, 14.2. HRMS (ESI): calcd for $\mathrm{C}_{16} \mathrm{H}_{22} \mathrm{NO}_{5}$ $[\mathrm{M}+\mathrm{H}]^{+}: 308.1498$, found 308.1505 .

\section{General procedure for the syntheses of imine $7^{[2]}$}

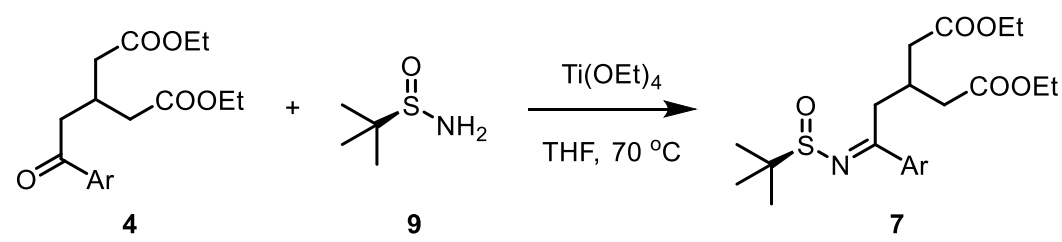

To a mixture of $(R)$-(-)-sulfinamide (133 $\mathrm{mg}, 1.1 \mathrm{mmol}, 1.1 \mathrm{equiv})$ and the corresponding ketone (376 mg, $1.0 \mathrm{mmol}, 1.0$ equiv) in THF (4 mL) was added $\mathrm{Ti}(\mathrm{OEt})_{4}$ (456 mg, $2.0 \mathrm{mmol}, 2.0$ equiv). The resulting mixture was heated at reflux in an oil bath. The reaction progress was monitored by TLC. Once complete, the reaction mixture was allowed to cool to room temperature. Brine $(4 \mathrm{~mL})$ was added to the reaction mixture with rapid stirring followed by the filtration through a pad of Celite®. The filtrate was dried over anhydrous $\mathrm{Na}_{2} \mathrm{SO}_{4}$ and concentrated under reduced pressure. The residue was purified by silica gel column chromatography to give the product.<smiles>CCOC(=O)CC(CC(=O)OCC)CC(=N[S@](=O)C(C)(C)C)c1ccc(C(F)(F)F)cc1</smiles>

Purification by silica gel column chromatography (ethyl acetate/petroleum ether $=1: 5$ ) to yield the desired product (339 mg, 71\% yield) as light yellow oil. ${ }^{1} \mathrm{H}$ NMR (400 MHz, Chloroform- $d$ ) $\delta 8.08(\mathrm{~d}, J=8.1 \mathrm{~Hz}, 2 \mathrm{H}), 7.70(\mathrm{~d}, J=8.2 \mathrm{~Hz}, 2 \mathrm{H}), 4.09(\mathrm{qd}, J=$ 7.1, $1.3 \mathrm{~Hz}, 4 \mathrm{H}), 3.66(\mathrm{dd}, J=13.3,8.0 \mathrm{~Hz}, 1 \mathrm{H}), 3.36(\mathrm{dd}, J=13.2,7.0 \mathrm{~Hz}, 1 \mathrm{H}), 2.80$ $-2.66(\mathrm{~m}, 1 \mathrm{H}), 2.58-2.44(\mathrm{~m}, 2 \mathrm{H}), 2.42-2.28(\mathrm{~m}, 2 \mathrm{H}), 1.32(\mathrm{~s}, 9 \mathrm{H}), 1.22(\mathrm{t}, J=7.1$ $\mathrm{Hz}, 6 \mathrm{H}) .{ }^{13} \mathrm{C}\left\{{ }^{1} \mathrm{H}\right\}$ NMR $(100 \mathrm{MHz}$, Chloroform- $d$ ) $\delta$ 176.1, 172.1, 171.9, 140.5, 133.0 
$(\mathrm{q}, J=32.7 \mathrm{~Hz}), 128.2,125.7(\mathrm{q}, J=4.3 \mathrm{~Hz}), 123.75(\mathrm{q}, J=272.3 \mathrm{~Hz}), 60.7,60.6,58.7$, 38.1, 37.9, 35.3, 31.0, 22.9, 14.2. ${ }^{19} \mathrm{~F}$ NMR (376 MHz, Chloroform- $d$ ) $\delta$-63.0. HRMS (ESI): calcd for $\mathrm{C}_{22} \mathrm{H}_{31} \mathrm{~F}_{3} \mathrm{NO}_{5} \mathrm{~S}[\mathrm{M}+\mathrm{H}]^{+}$: 478.1875, found 478.1886 .<smiles>CCOC(=O)CC(CC(=O)OCC)CC(=NC(C)(C)C)c1ccccc1</smiles>

Purification by silica gel column chromatography (ethyl acetate/petroleum ether $=1: 5$ ) to yield the desired product ( $258 \mathrm{mg}, 63 \%$ yield) as light yellow oil. ${ }^{1} \mathrm{H}$ NMR (400 MHz, Chloroform- $d$ ) $\delta 7.94(\mathrm{~d}, J=7.4 \mathrm{~Hz}, 2 \mathrm{H}), 7.54-7.37(\mathrm{~m}, 3 \mathrm{H}), 4.08(\mathrm{qd}, J=7.1$, $3.9 \mathrm{~Hz}, 4 \mathrm{H}), 3.59(\mathrm{dd}, J=13.5,7.8 \mathrm{~Hz}, 1 \mathrm{H}), 3.33(\mathrm{dd}, J=13.6,7.4 \mathrm{~Hz}, 1 \mathrm{H}), 2.86-$ $2.62(\mathrm{~m}, 1 \mathrm{H}), 2.60-2.43(\mathrm{~m}, 2 \mathrm{H}), 2.42-2.25(\mathrm{~m}, 2 \mathrm{H}), 1.31(\mathrm{~s}, 9 \mathrm{H}), 1.21(\mathrm{td}, J=7.1$, $2.3 \mathrm{~Hz}, 6 \mathrm{H}) .{ }^{13} \mathrm{C}\left\{{ }^{1} \mathrm{H}\right\} \mathrm{NMR}(100 \mathrm{MHz}$, Chloroform- $d) \delta 177.5,172.1,172.0,137.4$, 131.7, 128.6, 127.8, 60.5, 60.5, 58.2, 38.1, 37.9, 35.4, 31.2, 22.8, 14.2. HRMS (ESI): calcd for $\mathrm{C}_{21} \mathrm{H}_{32} \mathrm{NO}_{5} \mathrm{~S}[\mathrm{M}+\mathrm{H}]^{+}$: 410.2001 , found 410.1991 .<smiles>CCOC(=O)CC(CC(=O)OCC)CC(=NC(C)(C)C)c1cccc(F)c1</smiles>

Purification by silica gel column chromatography (ethyl acetate/petroleum ether = 1:5)to yield the desired product (299 mg, $70 \%$ yield) as light yellow oil. ${ }^{1} \mathrm{H}$ NMR (400 MHz, Chloroform- $d$ ) $\delta 7.76(\mathrm{~d}, J=7.8 \mathrm{~Hz}, 1 \mathrm{H}), 7.65(\mathrm{~d}, J=10.2 \mathrm{~Hz}, 1 \mathrm{H}), 7.42(\mathrm{td}, J$ $=8.0,5.8 \mathrm{~Hz}, 1 \mathrm{H}), 7.23-7.08(\mathrm{~m}, 1 \mathrm{H}), 4.24-4.01(\mathrm{~m}, 4 \mathrm{H}), 3.60(\mathrm{dd}, J=13.5,8.0 \mathrm{~Hz}$, $1 \mathrm{H}), 3.31(\mathrm{dd}, J=13.6,7.2 \mathrm{~Hz}, 1 \mathrm{H}), 2.88-2.65(\mathrm{~m}, 1 \mathrm{H}), 2.61-2.44(\mathrm{~m}, 2 \mathrm{H}), 2.44-$ $2.23(\mathrm{~m}, 2 \mathrm{H}), 1.32(\mathrm{~s}, 9 \mathrm{H}), 1.22(\mathrm{t}, J=7.1 \mathrm{~Hz}, 6 \mathrm{H}) .{ }^{13} \mathrm{C}\left\{{ }^{1} \mathrm{H}\right\}$ NMR $(100 \mathrm{MHz}$, Chloroform- $d$ ) $\delta 176.0,172.1,171.9,162.9(\mathrm{~d}, J=246.5 \mathrm{~Hz}), 139.7,130.2(\mathrm{~d}, J=8.0$ $\mathrm{Hz}), 123.6,118.6$ (d, $J=21.5 \mathrm{~Hz}), 114.6$ (d, $J=23.5 \mathrm{~Hz}), 60.6,60.6,58.5,38.1,38.0$, 35.3, 31.2, 22.9, 14.2. ${ }^{19} \mathrm{~F}$ NMR (376 MHz, Chloroform- $d$ ) $\delta$-112.0. HRMS (ESI): calcd for $\mathrm{C}_{21} \mathrm{H}_{31} \mathrm{FNO}_{5} \mathrm{~S}[\mathrm{M}+\mathrm{H}]^{+}$: 428.1907, found 428.1917 .<smiles>CCOC(=O)CC(CC(=O)NS(=O)C(C)(C)C)Cc1cccc(Cl)c1</smiles>

Purification by silica gel column chromatography (ethyl acetate/petroleum ether $=1: 5$ ) to yield the desired product (342 mg, 77\% yield) as light yellow oil. ${ }^{1} \mathrm{H}$ NMR (400 
MHz, Chloroform- $d$ ) $\delta 7.91(\mathrm{~s}, 1 \mathrm{H}), 7.85(\mathrm{~d}, J=7.8 \mathrm{~Hz}, 1 \mathrm{H}), 7.44(\mathrm{~d}, J=8.7 \mathrm{~Hz}, 1 \mathrm{H})$, $7.38(\mathrm{t}, J=7.8 \mathrm{~Hz}, 1 \mathrm{H}), 4.10(\mathrm{qd}, J=7.2,1.4 \mathrm{~Hz}, 4 \mathrm{H}), 3.60(\mathrm{dd}, J=13.5,8.0 \mathrm{~Hz}, 1 \mathrm{H})$, $3.30(\mathrm{dd}, J=13.5,7.1 \mathrm{~Hz}, 1 \mathrm{H}), 2.83-2.65(\mathrm{~m}, 1 \mathrm{H}), 2.56-2.45(\mathrm{~m}, 2 \mathrm{H}), 2.42-2.24$ $(\mathrm{m}, 2 \mathrm{H}), 1.31(\mathrm{~s}, 9 \mathrm{H}), 1.22(\mathrm{t}, J=7.1 \mathrm{~Hz}, 6 \mathrm{H}) .{ }^{13} \mathrm{C}\left\{{ }^{1} \mathrm{H}\right\}$ NMR $(100 \mathrm{MHz}$, Chloroformd) $\delta 175.9,172.0,171.9,139.1,134.8,131.6,129.9,127.8,126.0,60.6,60.6,58.5,38.1$, 37.9, 35.3, 31.1, 22.9, 22.8, 14.2. HRMS (ESI): calcd for $\mathrm{C}_{21} \mathrm{H}_{31} \mathrm{ClNO}_{5} \mathrm{~S}[\mathrm{M}+\mathrm{H}]^{+}$: 444.1611, found 444.1612 .<smiles>CCOC(=O)CC(CC(=O)OCC)CC(=NC(=O)C(C)(C)C)c1ccc(C)cc1</smiles>

Purification by silica gel column chromatography (ethyl acetate/petroleum ether $=1: 5$ ) to yield the desired product $\left(254 \mathrm{mg}, 60 \%\right.$ yield) as a light-yellow oil. ${ }^{1} \mathrm{H}$ NMR (400 MHz, Chloroform- $d$ ) $\delta 7.83(\mathrm{~d}, J=7.7 \mathrm{~Hz}, 2 \mathrm{H}), 7.21(\mathrm{~d}, J=8.0 \mathrm{~Hz}, 2 \mathrm{H}), 4.19-3.92$ (m, 4H), $3.54(\mathrm{dd}, J=13.6,8.0 \mathrm{~Hz}, 1 \mathrm{H}), 3.28(\mathrm{dd}, J=13.5,7.5 \mathrm{~Hz}, 1 \mathrm{H}), 2.88-2.63$ (m, 1H), $2.56-2.44(\mathrm{~m}, 2 \mathrm{H}), 2.43-2.22(\mathrm{~m}, 5 \mathrm{H}), 1.28(\mathrm{~s}, 9 \mathrm{H}), 1.24-1.00(\mathrm{~m}, 6 \mathrm{H})$. ${ }^{13} \mathrm{C}\left\{{ }^{1} \mathrm{H}\right\}$ NMR (100 MHz, Chloroform- $d$ ) $\delta 177.4,172.1,172.0,142.3,134.8,129.3$, $127.9,60.5,60.4,58.0,38.0,37.9,35.4,31.3,22.8,21.5,14.2$. HRMS (ESI): calcd for $\mathrm{C}_{22} \mathrm{H}_{34} \mathrm{NO}_{5} \mathrm{~S}[\mathrm{M}+\mathrm{H}]^{+}:$424.2158, found 424.2148 .<smiles>CCOC(=O)CC(CC(=O)OCC)CC(=N[S@](=O)C(C)(C)C)c1ccncc1</smiles>

Purification by silica gel column chromatography (ethyl acetate/petroleum ether $=1: 1$ ) to yield the desired product (320 mg, 78\% yield) as light yellow oil. ${ }^{1} \mathrm{H}$ NMR (400 MHz, Chloroform- $d$ ) $\delta 8.73(\mathrm{~d}, J=5.1 \mathrm{~Hz}, 2 \mathrm{H}), 7.79(\mathrm{~d}, J=5.1 \mathrm{~Hz}, 2 \mathrm{H}), 4.09$ (q, $J=$ $7.2 \mathrm{~Hz}, 4 \mathrm{H}), 3.63(\mathrm{dd}, J=13.4,8.1 \mathrm{~Hz}, 1 \mathrm{H}), 3.31(\mathrm{dd}, J=13.4,7.0 \mathrm{~Hz}, 1 \mathrm{H}), 2.81-$ $2.61(\mathrm{~m}, 1 \mathrm{H}), 2.49(\mathrm{dt}, J=16.4,6.7 \mathrm{~Hz}, 2 \mathrm{H}), 2.34(\mathrm{dt}, J=16.7,8.0 \mathrm{~Hz}, 2 \mathrm{H}), 1.32(\mathrm{~s}$, 9H), $1.21(\mathrm{t}, J=7.2 \mathrm{~Hz}, 6 \mathrm{H}) .{ }^{13} \mathrm{C}\left\{{ }^{1} \mathrm{H}\right\}$ NMR (100 MHz, Chloroform- $\left.d\right) \delta 175.3,172.0$, $171.8,150.7,144.2,121.2,60.7,60.6,59.0,38.2$, 38.0, 34.9, 30.9, 23.0, 14.2. HRMS (ESI): calcd for $\mathrm{C}_{20} \mathrm{H}_{31} \mathrm{~N}_{2} \mathrm{O}_{5} \mathrm{~S}[\mathrm{M}+\mathrm{H}]^{+}:$411.1954, found 411.1954 .

\section{General procedure for the imine reduction}

Table 1. Optimization of reaction conditions 


\begin{tabular}{|c|c|c|c|}
\hline Entry & Conditions & Yield $(\%)^{\mathrm{a}}$ & $8 \mathrm{a}: 8 \mathrm{a}^{\prime}$ \\
\hline 1 & $\begin{array}{c}\mathrm{NaBH}_{4}, \mathrm{MeOH} \\
\mathrm{THF},-20^{\circ} \mathrm{C}\end{array}$ & 85 & $1: 3$ \\
\hline 2 & $\begin{array}{c}\text { DIBAL-H } \\
\text { PhMe, }-78^{\circ} \mathrm{C}\end{array}$ & 38 & $<1: 10$ \\
\hline 3 & $\begin{array}{c}\mathrm{LiBHEt}_{3} \\
\mathrm{THF},-78{ }^{\circ} \mathrm{C}\end{array}$ & 75 & $>10: 1$ \\
\hline 4 & $\begin{array}{l}\text { L-Selectride } \\
\text { THF, }-78{ }^{\circ} \mathrm{C}\end{array}$ & 83 & $>10: 1$ \\
\hline 5 & $\begin{array}{l}\mathrm{Pd} / \mathrm{C}, \mathrm{H}_{2} \\
\mathrm{EtOH}, \mathrm{rt}\end{array}$ & NR & -- \\
\hline
\end{tabular}

${ }^{a}$ Isolated yield after silica gel chromatography.

\section{General procedure for the syntheses of $8^{[3]}$}<smiles>CCOC(=O)CC(CC(=O)S(=O)C(C)(C)C)CC(=[V])OCC</smiles>

7

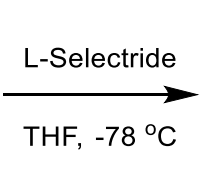
L-selectride (1.0 $\mathrm{M}$ in THF, $3.8 \mathrm{~mL}, 3.8 \mathrm{mmol}, 1.2$ equiv) was added to a cooled
solution of $7\left(3.1 \mathrm{mmol}, 1.0\right.$ equiv) in THF $(13 \mathrm{~mL})$ at $-78^{\circ} \mathrm{C}$. The reaction mixture was stirred at $-78{ }^{\circ} \mathrm{C}$ for $12 \mathrm{~h}$. The reaction was quenched with $\mathrm{NH}_{4} \mathrm{Cl}$ aq. and extracted with EtOAc $(40 \mathrm{~mL})$. The organic layer was dried over $\mathrm{Na}_{2} \mathrm{SO}_{4}$ and concentrated in vacuo. The residue was purified by silica gel column chromatography to give the product.<smiles>CCOC(=O)CC(CC(=O)OCC)CC(NS(=O)C(C)(C)C)c1ccc(C(F)(F)F)cc1</smiles>

Purification by silica gel column chromatography (ethyl acetate/petroleum ether $=1: 1$ ) to yield the desired product $(1.23 \mathrm{~g}, 83 \%$ yield $)$ as light yellow oil. ${ }^{1} \mathrm{H}$ NMR $(400 \mathrm{MHz}$, Chloroform- $d$ ) $\delta 7.58(\mathrm{~d}, J=8.1 \mathrm{~Hz}, 2 \mathrm{H}), 7.43(\mathrm{~d}, J=8.0 \mathrm{~Hz}, 2 \mathrm{H}), 4.54(\mathrm{td}, J=6.8,2.1$ 
$\mathrm{Hz}, 1 \mathrm{H}), 4.32(\mathrm{~d}, J=2.2 \mathrm{~Hz}, 1 \mathrm{H}), 4.20-3.99(\mathrm{~m}, 4 \mathrm{H}), 2.53-2.24(\mathrm{~m}, 5 \mathrm{H}), 1.83(\mathrm{dd}$, $J=7.1,5.0 \mathrm{~Hz}, 2 \mathrm{H}), 1.29-1.14(\mathrm{~m}, 15 \mathrm{H}) .{ }^{13} \mathrm{C}\left\{{ }^{1} \mathrm{H}\right\}$ NMR $(100 \mathrm{MHz}$, Chloroform- $d) \delta$ $172.5,172.0,146.7,129.8(\mathrm{q}, J=32.4 \mathrm{~Hz}), 127.6,125.5(\mathrm{q}, J=3.6 \mathrm{~Hz}), 124.1(\mathrm{q}, J=$ $272.1 \mathrm{~Hz}), 60.7,60.6,56.4,55.8,42.2,38.3,38.0,28.8,22.7,14.2 .{ }^{19} \mathrm{~F}$ NMR $(376 \mathrm{MHz}$, Chloroform- $d$ ) $\delta-62.5$. HRMS (ESI): calcd for $\mathrm{C}_{22} \mathrm{H}_{33} \mathrm{~F}_{3} \mathrm{NO}_{5} \mathrm{~S}[\mathrm{M}+\mathrm{H}]^{+}:$480.2032, found 480.2043 .<smiles>CCOC(=O)CC(CC(=O)OCC)C[C@H](N[S@](=O)C(C)(C)C)c1ccc(C(F)(F)F)cc1</smiles>

Reduced by $\mathrm{NaBH}_{4}$; Light yellow oil. ${ }^{1} \mathrm{H} \mathrm{NMR}(400 \mathrm{MHz}$, Chloroform- $d$ ) $\delta 7.61$ (d, $J$ $=8.1 \mathrm{~Hz}, 2 \mathrm{H}), 7.47(\mathrm{~d}, J=8.1 \mathrm{~Hz}, 2 \mathrm{H}), 4.48(\mathrm{dt}, J=8.2,6.5 \mathrm{~Hz}, 1 \mathrm{H}), 4.23-4.03(\mathrm{~m}$, $4 \mathrm{H}), 3.71(\mathrm{~d}, J=6.4 \mathrm{~Hz}, 1 \mathrm{H}), 2.52-2.26(\mathrm{~m}, 5 \mathrm{H}), 2.06-1.91(\mathrm{~m}, 1 \mathrm{H}), 1.89-1.73(\mathrm{~m}$, $1 \mathrm{H}), 1.34-1.14(\mathrm{~m}, 15 \mathrm{H}) .{ }^{13} \mathrm{C}\left\{{ }^{1} \mathrm{H}\right\} \mathrm{NMR}(100 \mathrm{MHz}$, Chloroform- $d$ ) $\delta$ 172.1, 172.0, 146.4, 130.1 (q, $J=32.7 \mathrm{~Hz}), 127.4,125.8(\mathrm{q}, J=3.7 \mathrm{~Hz}), 124.0(\mathrm{q}, J=272.2 \mathrm{~Hz})$, 60.6, 60.6, 41.4 38.2 , 38.1, 28.7, 22.6, 14.2, 14.2. ${ }^{19} \mathrm{~F}$ NMR (376 MHz, Chloroform- $d$ ) $\delta$-62.6. HRMS (ESI): calcd for $\mathrm{C}_{22} \mathrm{H}_{33} \mathrm{~F}_{3} \mathrm{NO}_{5} \mathrm{~S}[\mathrm{M}+\mathrm{H}]^{+}:$480.2032, found 480.2038.<smiles>CCOC(=O)CC(CC(=O)OCC)c1ccccc1</smiles>

$8 b$

Purification by silica gel column chromatography (ethyl acetate/petroleum ether $=1: 1$ ) to yield the desired product ( $829 \mathrm{mg}, 65 \%$ yield) as light yellow oil. ${ }^{1} \mathrm{H}$ NMR (400 $\mathrm{MHz}$, Chloroform- $d$ ) $\delta 7.39-7.23(\mathrm{~m}, 5 \mathrm{H}), 4.48(\mathrm{t}, J=7.1 \mathrm{~Hz}, 1 \mathrm{H}), 4.21-3.95(\mathrm{~m}$, $5 \mathrm{H}), 2.53-2.25(\mathrm{~m}, 5 \mathrm{H}), 1.85(\mathrm{t}, J=6.1 \mathrm{~Hz}, 2 \mathrm{H}), 1.34-1.14(\mathrm{~m}, 15 \mathrm{H}) .{ }^{13} \mathrm{C}\left\{{ }^{1} \mathrm{H}\right\} \mathrm{NMR}$ (100 MHz, Chloroform- $d$ ) $\delta$ 172.4, 172.0, 142.2, 128.5, 127.6, 127.3, 60.5, 60.5, 56.8, 55.6, 42.4, 38.4, 37.9, 28.9, 22.7, 14.2. HRMS (ESI): calcd for $\mathrm{C}_{21} \mathrm{H}_{34} \mathrm{NO}_{5} \mathrm{~S}[\mathrm{M}+\mathrm{H}]^{+}$: 412.2158, found 412.2163 .<smiles>CCOC(=O)CC(CC(=O)OCC)CC(NS(=O)C(C)(C)C)c1cccc(F)c1</smiles>

Purification by silica gel column chromatography (ethyl acetate/petroleum ether $=1: 1$ ) to yield the desired product (945 mg, 71\% yield) as light yellow oil; ${ }^{1} \mathrm{H}$ NMR (400 MHz, Chloroform- $d$ ) $\delta 7.31-7.22(\mathrm{~m}, 1 \mathrm{H}), 7.07(\mathrm{dt}, J=7.6,1.2 \mathrm{~Hz}, 1 \mathrm{H}), 7.01(\mathrm{dt}, J=$ 9.9, $2.1 \mathrm{~Hz}, 1 \mathrm{H}), 6.93(\mathrm{tdd}, J=8.4,2.7,1.0 \mathrm{~Hz}, 1 \mathrm{H}), 4.46(\mathrm{td}, J=6.8,2.1 \mathrm{~Hz}, 1 \mathrm{H}), 4.21$ 
$(\mathrm{d}, J=2.3 \mathrm{~Hz}, 1 \mathrm{H}), 4.16-4.02(\mathrm{~m}, 4 \mathrm{H}), 2.56-2.24(\mathrm{~m}, 5 \mathrm{H}), 1.80(\mathrm{t}, J=6.0 \mathrm{~Hz}, 2 \mathrm{H})$, $1.23(\mathrm{td}, J=7.1,4.3 \mathrm{~Hz}, 6 \mathrm{H}), 1.20(\mathrm{~s}, 9 \mathrm{H}) .{ }^{13} \mathrm{C}\left\{{ }^{1} \mathrm{H}\right\}$ NMR (100 MHz, Chloroform- $\left.d\right) \delta$ $172.4,172.0,163.0(\mathrm{~d}, J=246.3 \mathrm{~Hz}), 145.2(\mathrm{~d}, J=6.7 \mathrm{~Hz}), 130.1(\mathrm{~d}, J=8.5 \mathrm{~Hz})$, $123.0(\mathrm{~d}, J=2.9 \mathrm{~Hz}), 114.5(\mathrm{~d}, J=21.1 \mathrm{~Hz}), 114.0(\mathrm{~d}, J=22.0 \mathrm{~Hz}), 60.6,60.6,56.3$, 55.7 , 42.2 , 38.3 , 38.0 , 28.8 , 22.7 , 14.2. ${ }^{19} \mathrm{~F}$ NMR (376 MHz, Chloroform- $d$ ) $\delta$-112.7. HRMS (ESI): calcd for $\mathrm{C}_{21} \mathrm{H}_{33} \mathrm{FNO}_{5} \mathrm{~S}[\mathrm{M}+\mathrm{H}]^{+}:$430.2063, found 430.2061.<smiles>CCOC(=O)CC(CC(=O)OCC)CC(NS(=O)C(C)(C)C)c1cccc(Cl)c1</smiles>

Purification by silica gel column chromatography (ethyl acetate/petroleum ether $=1: 1$ ) to yield the desired product (995 mg, 72\% yield) as light yellow oil. ${ }^{1} \mathrm{H}$ NMR (400 MHz, Chloroform- $d$ ) $\delta 7.29(\mathrm{~d}, J=2.0 \mathrm{~Hz}, 1 \mathrm{H}), 7.28-7.20(\mathrm{~m}, 2 \mathrm{H}), 7.20-7.15(\mathrm{~m}$, $1 \mathrm{H}), 4.44(\mathrm{t}, J=6.8 \mathrm{~Hz}, 1 \mathrm{H}), 4.23(\mathrm{~d}, J=2.5 \mathrm{~Hz}, 1 \mathrm{H}), 4.17-4.04(\mathrm{~m}, 4 \mathrm{H}), 2.50-2.25$ $(\mathrm{m}, 5 \mathrm{H}), 1.81(\mathrm{t}, J=5.8 \mathrm{~Hz}, 2 \mathrm{H}), 1.33-1.11(\mathrm{~m}, 15 \mathrm{H}) .{ }^{13} \mathrm{C}\left\{{ }^{1} \mathrm{H}\right\}$ NMR $(100 \mathrm{MHz}$, $\left.\mathrm{CDCl}_{3}\right) \delta 172.4,171.9,144.7,134.4,129.8,127.8,127.3,125.5,60.6,60.6,56.3,55.7$, 42.2, 38.3, 38.0, 28.9, 22.7, 14.2. HRMS (ESI): calcd for $\mathrm{C}_{21} \mathrm{H}_{33} \mathrm{ClNO}_{5} \mathrm{~S}[\mathrm{M}+\mathrm{H}]^{+}$: 446.1768 , found 446.1768 .<smiles>CCOC(=O)CC(CC(=O)OCC)Cc1ccc(C)cc1</smiles>

$8 \mathrm{e}$

Purification by silica gel column chromatography (ethyl acetate/petroleum ether $=1: 1$ ) to yield the desired product (792 mg, 60\% yield) as light yellow oil. ${ }^{1} \mathrm{H}$ NMR (400 MHz, Chloroform- $d$ ) $\delta 7.17(\mathrm{~d}, J=7.8 \mathrm{~Hz}, 2 \mathrm{H}), 7.11(\mathrm{~d}, J=7.8 \mathrm{~Hz}, 2 \mathrm{H}), 4.50-4.34$ $(\mathrm{m}, 1 \mathrm{H}), 4.17-4.03(\mathrm{~m}, 4 \mathrm{H}), 4.00(\mathrm{~s}, 1 \mathrm{H}), 2.49-2.21(\mathrm{~m}, 5 \mathrm{H}), 2.30(\mathrm{~s}, 3 \mathrm{H}), 1.81(\mathrm{t}, J$ $=6.3 \mathrm{~Hz}, 2 \mathrm{H}), 1.32-1.06(\mathrm{~m}, 15 \mathrm{H}) .{ }^{13} \mathrm{C}\left\{{ }^{1} \mathrm{H}\right\}$ NMR $(100 \mathrm{MHz}$, Chloroform- $d) \delta 172.3$, 172.1, 139.0, 137.3, 129.2, 127.3, 60.5, 60.4, 56.5, 55.5, 42.4, 38.4, 37.9, 28.9, 22.6, 21.1, 14.2, 14.2. HRMS (ESI): calcd for $\mathrm{C}_{22} \mathrm{H}_{36} \mathrm{NO}_{5} \mathrm{~S}[\mathrm{M}+\mathrm{H}]^{+}: 426.2314$, found 426.2319 .<smiles>CCOC(=O)CC(CC(=O)OCC)c1ccncc1</smiles>

Purification by silica gel column chromatography $(\mathrm{DCM} / \mathrm{MeOH}=20: 1)$ to yield the desired product $\left(857 \mathrm{mg}, 67 \%\right.$ yield) as light yellow oil. ${ }^{1} \mathrm{H}$ NMR (400 MHz, Chloroform- $d$ ) $\delta 8.62-8.50(\mathrm{~m}, 2 \mathrm{H}), 7.32-7.26(\mathrm{~m}, 2 \mathrm{H}), 4.57-4.41(\mathrm{~m}, 2 \mathrm{H}), 4.22-$ 
$3.98(\mathrm{~m}, 4 \mathrm{H}), 2.57-2.21(\mathrm{~m}, 5 \mathrm{H}), 1.79(\mathrm{t}, J=6.0 \mathrm{~Hz}, 2 \mathrm{H}), 1.38-1.11(\mathrm{~m}, 15 \mathrm{H})$. ${ }^{13} \mathrm{C}\left\{{ }^{1} \mathrm{H}\right\}$ NMR (100 MHz, Chloroform- $d$ ) $\delta 172.5,171.9,152.8,149.7,122.5,60.7$, 60.7, 56.0, 41.7, 38.2, 38.0, 28.8, 22.7, 14.2, 14.2. HRMS (ESI): calcd for $\mathrm{C}_{20} \mathrm{H}_{33} \mathrm{~N}_{2} \mathrm{O}_{5} \mathrm{~S}$ $[\mathrm{M}+\mathrm{H}]^{+}:$413.2110, found 413.2119.

\section{General procedure for the syntheses of lactam 6}

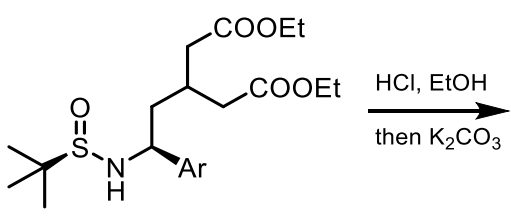

8<smiles>COCCC1CC(=O)NC([AlH2])C1</smiles>

6

To a solution of 8 ( $0.3 \mathrm{mmol}, 1.0$ equiv) in EtOH ( $3 \mathrm{~mL})$ was added a solution of $2 \mathrm{M}$ $\mathrm{HCl}$ in $\mathrm{EtOH}\left(0.45 \mathrm{~mL}, 0.9 \mathrm{mmol}, 3.0\right.$ equiv) at $0{ }^{\circ} \mathrm{C}$. The mixture was moved to room temperature and stirred for $1 \mathrm{~h}$. The solvent was removed under reduced pressure. The residue was dissolved in $\mathrm{EtOH}(3 \mathrm{~mL})$, and then $\mathrm{K}_{2} \mathrm{CO}_{3}(414 \mathrm{mg}, 3.0 \mathrm{mmol}, 10.0$ equiv) was added. The reaction mixture was allowed to stir for $12 \mathrm{~h}$ at room temperature before filtration through a pad of Celite $\AA$. The filtrate was concentrated under reduced pressure and purified by silica gel column chromatography to give the product.<smiles>CCOCCC1CC(=O)NC(c2ccc(C(F)(F)F)cc2)C1</smiles>

Purification by silica gel column chromatography $(\mathrm{DCM} / \mathrm{MeOH}=15: 1)$ to yield the desired product (89 mg, $90 \%$ yield) as white solid. ${ }^{1} \mathrm{H}$ NMR $(400 \mathrm{MHz}$, Chloroform- $d$ ) $\delta 7.64(\mathrm{~d}, J=7.9 \mathrm{~Hz}, 2 \mathrm{H}), 7.44(\mathrm{~d}, J=7.9 \mathrm{~Hz}, 2 \mathrm{H}), 5.93(\mathrm{~s}, 1 \mathrm{H}), 4.63(\mathrm{dd}, J=11.6,4.1$ $\mathrm{Hz}, 1 \mathrm{H}), 4.14$ (q, $J=7.2 \mathrm{~Hz}, 2 \mathrm{H}), 2.63$ (ddd, $J=17.2,4.9,2.3 \mathrm{~Hz}, 1 \mathrm{H}), 2.57-2.44$ (m, $1 \mathrm{H}), 2.44-2.25(\mathrm{~m}, 2 \mathrm{H}), 2.24-2.12(\mathrm{~m}, 2 \mathrm{H}), 1.39(\mathrm{q}, J=12.3 \mathrm{~Hz}, 1 \mathrm{H}), 1.26(\mathrm{t}, J=$ 7.2, $1.3 \mathrm{~Hz}, 3 \mathrm{H}) .{ }^{13} \mathrm{C}\left\{{ }^{1} \mathrm{H}\right\}$ NMR $(100 \mathrm{MHz}$, Chloroform- $d) \delta 171.5,171.4,145.9$, $130.5(\mathrm{q}, J=32.6 \mathrm{~Hz}), 126.5,126.0(\mathrm{q}, J=3.7 \mathrm{~Hz}), 123.9(\mathrm{q}, J=272.1 \mathrm{~Hz}), 60.7$, 57.3 , 40.1 , 38.7 , 37.3 , 30.0 , 14.2.${ }^{19} \mathrm{~F} \mathrm{NMR}$ (376 MHz, Chloroform-d) $\delta$-62.6. HRMS (ESI): calcd for $\mathrm{C}_{16} \mathrm{H}_{19} \mathrm{~F}_{3} \mathrm{NO}_{3}[\mathrm{M}+\mathrm{H}]^{+}: 330.1317$, found 330.1324 .<smiles>CCOCCC1CC(=O)NC(c2ccccc2)C1</smiles>

Purification by silica gel column chromatography $(\mathrm{DCM} / \mathrm{MeOH}=15: 1)$ to yield the desired product (69 mg, 88\% yield) as white solid. ${ }^{1} \mathrm{H}$ NMR (400 MHz, Chloroform- $d$ ) $\delta 7.46-7.22(\mathrm{~m}, 5 \mathrm{H}), 5.88(\mathrm{~s}, 1 \mathrm{H}), 4.54(\mathrm{dd}, J=11.6,4.0 \mathrm{~Hz}, 1 \mathrm{H}), 4.13$ (q, $J=7.2 \mathrm{~Hz}$, $2 \mathrm{H}), 2.62(\mathrm{dd}, J=17.8,4.5 \mathrm{~Hz}, 1 \mathrm{H}), 2.57-2.41(\mathrm{~m}, 1 \mathrm{H}), 2.41-2.25(\mathrm{~m}, 2 \mathrm{H}), 2.25-$ 
$2.08(\mathrm{~m}, 2 \mathrm{H}), 1.41(\mathrm{q}, J=12.4 \mathrm{~Hz}, 1 \mathrm{H}), 1.26(\mathrm{t}, J=7.1 \mathrm{~Hz}, 3 \mathrm{H}) .{ }^{13} \mathrm{C}\left\{{ }^{1} \mathrm{H}\right\}$ NMR $(100$ MHz, Chloroform- $d$ ) $\delta$ 171.5, 171.3, 141.9, 129.0, 128.3, 126.1, 60.7, 57.7, 40.3, 38.9, 37.3, 30.1, 14.2. HRMS (ESI): calcd for $\mathrm{C}_{15} \mathrm{H}_{20} \mathrm{NO}_{3}[\mathrm{M}+\mathrm{H}]^{+}: 262.1443$, found 262.1445 .

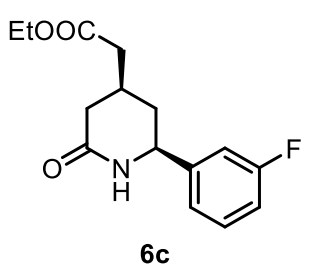

Purification by silica gel column chromatography $(\mathrm{DCM} / \mathrm{MeOH}=15: 1)$ to yield the desired product ( $80 \mathrm{mg}, 95 \%$ yield) as white solid. ${ }^{1} \mathrm{H}$ NMR (400 MHz, Chloroform- $d$ ) $\delta 7.36-7.27(\mathrm{~m}, 1 \mathrm{H}), 7.06(\mathrm{~d}, J=7.7 \mathrm{~Hz}, 1 \mathrm{H}), 7.03-6.90(\mathrm{~m}, 2 \mathrm{H}), 6.31(\mathrm{~s}, 1 \mathrm{H}), 4.52$ $(\mathrm{dd}, J=11.6,4.1 \mathrm{~Hz}, 1 \mathrm{H}), 4.11(\mathrm{q}, J=7.2 \mathrm{~Hz}, 2 \mathrm{H}), 2.56(\mathrm{ddd}, J=17.2,4.9,2.4 \mathrm{~Hz}$, $1 \mathrm{H}), 2.51-2.37(\mathrm{~m}, 1 \mathrm{H}), 2.36-2.23(\mathrm{~m}, 2 \mathrm{H}), 2.19-2.05(\mathrm{~m}, 2 \mathrm{H}), 1.35$ (q, $J=12.3$ $\mathrm{Hz}, 1 \mathrm{H}), 1.23(\mathrm{t}, J=7.1,3 \mathrm{H}) .{ }^{13} \mathrm{C}\left\{{ }^{1} \mathrm{H}\right\}$ NMR $(100 \mathrm{MHz}$, Chloroform- $d) \delta 171.5,171.4$, $163.0(\mathrm{~d}, J=247.2 \mathrm{~Hz}), 144.6(\mathrm{~d}, J=7.0 \mathrm{~Hz}), 130.6(\mathrm{~d}, J=8.5 \mathrm{~Hz}), 121.7$ (d, $J=3.0$ $\mathrm{Hz}), 115.1(\mathrm{~d}, J=21.1 \mathrm{~Hz}), 113.1(\mathrm{~d}, J=22.1 \mathrm{~Hz}), 60.7$, 40.2 , 38.7 , 37.3 , 29.9 , 14.2. ${ }^{19} \mathrm{~F}$ NMR (376 MHz, Chloroform-d) $\delta$-111.9. HRMS (ESI): calcd for $\mathrm{C}_{15} \mathrm{H}_{19} \mathrm{FNO}_{3}$ $[\mathrm{M}+\mathrm{H}]^{+}: 280.1349$, found 280.1348 .

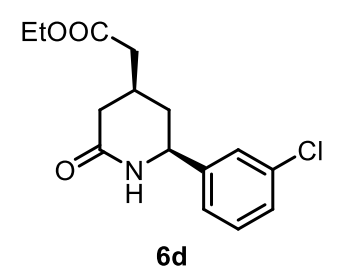

Purification by silica gel column chromatography $(\mathrm{DCM} / \mathrm{MeOH}=15: 1)$ to yield the desired product ( $81 \mathrm{mg}, 91 \%$ yield) as colorless oil. ${ }^{1} \mathrm{H}$ NMR (400 MHz, Chloroformd) $\delta 7.33-7.24(\mathrm{~m}, 3 \mathrm{H}), 7.16(\mathrm{dt}, J=6.6,2.1 \mathrm{~Hz}, 1 \mathrm{H}), 6.30(\mathrm{~s}, 1 \mathrm{H}), 4.51(\mathrm{dd}, J=11.5$, $4.2 \mathrm{~Hz}, 1 \mathrm{H}), 4.12(\mathrm{q}, J=7.1 \mathrm{~Hz}, 2 \mathrm{H}), 2.57(\mathrm{ddd}, J=17.2,5.0,2.3 \mathrm{~Hz}, 1 \mathrm{H}), 2.51-2.38$ $(\mathrm{m}, 1 \mathrm{H}), 2.37-2.22(\mathrm{~m}, 2 \mathrm{H}), 2.10(\mathrm{td}, J=17.3,15.5,10.9 \mathrm{~Hz}, 2 \mathrm{H}), 1.35(\mathrm{dt}, J=13.2$, $11.8 \mathrm{~Hz}, 1 \mathrm{H}), 1.23(\mathrm{t}, J=7.1 \mathrm{~Hz}, 3 \mathrm{H}) .{ }^{13} \mathrm{C}\left\{{ }^{1} \mathrm{H}\right\}$ NMR $(100 \mathrm{MHz}$, Chloroform- $d) \delta 171.5$, 171.4, 144.0, 134.8, 130.3, 128.4, 126.3, 124.3, 60.7, 57.1, 40.1, 38.7, 37.2, 29.9, 14.2. HRMS (ESI): calcd for $\mathrm{C}_{15} \mathrm{H}_{19} \mathrm{ClNO}_{3}[\mathrm{M}+\mathrm{H}]^{+}:$296.1053, found 296.1048 .

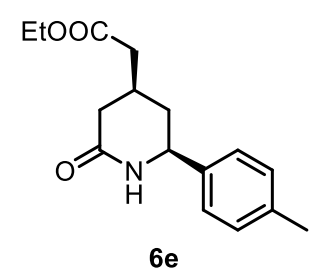

Purification by silica gel column chromatography $(\mathrm{DCM} / \mathrm{MeOH}=15: 1)$ to yield the desired product (74 mg, 90\% yield) as white solid. ${ }^{1} \mathrm{H}$ NMR $(400 \mathrm{MHz}$, Chloroform- $d$ ) $\delta 7.22-7.12(\mathrm{~m}, 4 \mathrm{H}), 4.50(\mathrm{dd}, J=11.6,4.0 \mathrm{~Hz}, 1 \mathrm{H}), 4.14(\mathrm{q}, J=7.2 \mathrm{~Hz}, 2 \mathrm{H}), 2.61$ (ddd, $J=17.4,4.8,2.3 \mathrm{~Hz}, 1 \mathrm{H}), 2.55-2.40(\mathrm{~m}, 1 \mathrm{H}), 2.34(\mathrm{~s}, 3 \mathrm{H}), 2.33(\mathrm{~d}, J=9.2 \mathrm{~Hz}$, 
$2 \mathrm{H}), 2.12(\mathrm{td}, J=11.5,6.6 \mathrm{~Hz}, 2 \mathrm{H}), 1.40(\mathrm{q}, J=12.4 \mathrm{~Hz}, 1 \mathrm{H}), 1.25(\mathrm{t}, J=7.1 \mathrm{~Hz}, 3 \mathrm{H})$. ${ }^{13} \mathrm{C}\left\{{ }^{1} \mathrm{H}\right\}$ NMR $(100 \mathrm{MHz}$, Chloroform- $d) \delta 171.5,171.4,138.9,138.1,129.6,126.0$, 60.7, 57.4, 40.3, 38.9, 37.3, 30.0, 21.1, 14.2. HRMS (ESI): calcd for $\mathrm{C}_{16} \mathrm{H}_{22} \mathrm{NO}_{3}$ $[\mathrm{M}+\mathrm{H}]^{+}: 276.1600$, found 276.1604 .

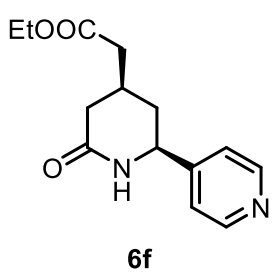

Purification by silica gel column chromatography $(\mathrm{DCM} / \mathrm{MeOH}=10: 1)$ to yield the desired product ( $68 \mathrm{mg}, 86 \%$ yield) as colorless oil. ${ }^{1} \mathrm{H}$ NMR (400 MHz, Chloroformd) $\delta 8.59(\mathrm{~d}, J=5.1 \mathrm{~Hz}, 2 \mathrm{H}), 7.24(\mathrm{~d}, J=4.8 \mathrm{~Hz}, 2 \mathrm{H}), 6.50(\mathrm{~s}, 1 \mathrm{H}), 4.55(\mathrm{dd}, J=11.6$, $4.2 \mathrm{~Hz}, 1 \mathrm{H}), 4.12(\mathrm{q}, J=7.1 \mathrm{~Hz}, 2 \mathrm{H}), 2.67-2.54(\mathrm{~m}, 1 \mathrm{H}), 2.54-2.41(\mathrm{~m}, 1 \mathrm{H}), 2.39-$ $2.23(\mathrm{~m}, 2 \mathrm{H}), 2.23-2.00(\mathrm{~m}, 2 \mathrm{H}), 1.33(\mathrm{q}, J=12.4 \mathrm{~Hz}, 1 \mathrm{H}), 1.24(\mathrm{t}, J=7.1 \mathrm{~Hz}, 3 \mathrm{H})$. ${ }^{13} \mathrm{C}\left\{{ }^{1} \mathrm{H}\right\}$ NMR (100 MHz, Chloroform- $d$ ) $\delta$ 171.6, 171.3, 151.0, 150.3, 121.0, 60.7, 56.6, 40.0, 38.2, 37.3, 29.9, 14.2. HRMS (ESI): calcd for $\mathrm{C}_{14} \mathrm{H}_{19} \mathrm{~N}_{2} \mathrm{O}_{3}[\mathrm{M}+\mathrm{H}]^{+}$: 263.1396, found 263.1395 .

\section{Synthesis of GSM-1}

Reduction of the lactam $6 \mathrm{a}^{[4]}$<smiles>CCOC(=O)CC1CC(=O)NC(c2ccc(C(F)(F)F)cc2)C1</smiles><smiles>COC(=O)CC1CCNC(c2ccc(C(F)(F)F)cc2)C1</smiles>

To a solution of $\mathbf{6 a}\left(300 \mathrm{mg}, 0.91 \mathrm{mmol}, 1.0\right.$ equiv) in THF (9 mL) under a $\mathrm{N}_{2}$ atmosphere was added $\mathrm{BH}_{3}-\mathrm{Me}_{2} \mathrm{~S}(1.37 \mathrm{~mL}$ of $2 \mathrm{M}$ in THF, $2.74 \mathrm{mmol}, 3.0$ equiv) at $0{ }^{\circ} \mathrm{C}$. The reaction mixture was stirred at $0{ }^{\circ} \mathrm{C}$ for $12 \mathrm{~h}$. EtOH $(2 \mathrm{~mL})$ was added slowly to quench the reaction. The solvent was removed under reduced pressure. The residue was dissolved in $\mathrm{EtOH}(5 \mathrm{~mL})$ followed by the addition of a solution of $2 \mathrm{M} \mathrm{HCl}$ in $\mathrm{EtOH}(5 \mathrm{~mL})$. The reaction mixture was allowed to stir for $1 \mathrm{~h}$ at $50{ }^{\circ} \mathrm{C}$ in an oil bath, then cooled to room temperature. The solvent was removed under reduced pressure. The residue was dissolved in Water $(15 \mathrm{~mL})$ and washed with DCM. The aqueous layer was basified with saturated $\mathrm{K}_{2} \mathrm{CO}_{3}$ aq. to $\mathrm{PH} \approx 11$, and extracted with DCM. The combined organic layers were concentrated under reduced pressure. The product was pure enough without the need for further purification (255 mg, 89\%). 


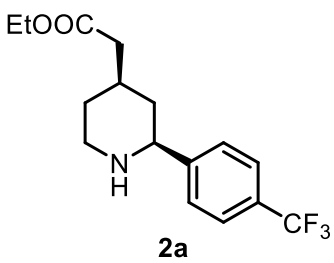

Colorless oil; ${ }^{1} \mathrm{H}$ NMR (400 MHz, Chloroform- $d$ ) $\delta 7.56(\mathrm{~d}, J=8.1 \mathrm{~Hz}, 2 \mathrm{H}), 7.47(\mathrm{~d}, J$ $=8.0 \mathrm{~Hz}, 2 \mathrm{H}), 4.11(\mathrm{q}, J=7.2 \mathrm{~Hz}, 2 \mathrm{H}), 3.71(\mathrm{dd}, J=11.3,2.4 \mathrm{~Hz}, 1 \mathrm{H}), 3.22(\mathrm{ddd}, J=$ $11.8,4.2,2.5 \mathrm{~Hz}, 1 \mathrm{H}), 2.84$ (td, $J=12.0,2.6 \mathrm{~Hz}, 1 \mathrm{H}), 2.38-2.17(\mathrm{~m}, 2 \mathrm{H}), 2.14-1.99$ $(\mathrm{m}, 1 \mathrm{H}), 1.92-1.82(\mathrm{~m}, 1 \mathrm{H}), 1.82-1.67(\mathrm{~m}, 2 \mathrm{H}), 1.38-1.01(\mathrm{~m}, 5 \mathrm{H}) .{ }^{13} \mathrm{C}\left\{{ }^{1} \mathrm{H}\right\} \mathrm{NMR}$ $(100 \mathrm{MHz}$, Chloroform- $d$ ) $\delta 172.5,148.9$, 129.4 (q, $J=32.3 \mathrm{~Hz}), 127.0,125.4$ (q, $J$ $=3.9 \mathrm{~Hz}), 124.2(\mathrm{q}, J=271.7 \mathrm{~Hz}), 61.2,60.3$, 46.8 , 41.6 , 41.2 , 33.9 , 32.0 , 14.3. HRMS (ESI): calcd for $\mathrm{C}_{16} \mathrm{H}_{21} \mathrm{~F}_{3} \mathrm{NO}_{2}[\mathrm{M}+\mathrm{H}]^{+}: 316.1524$, found 316.1527 .

Compound 12 was made following to the known procedure. ${ }^{[5]}$
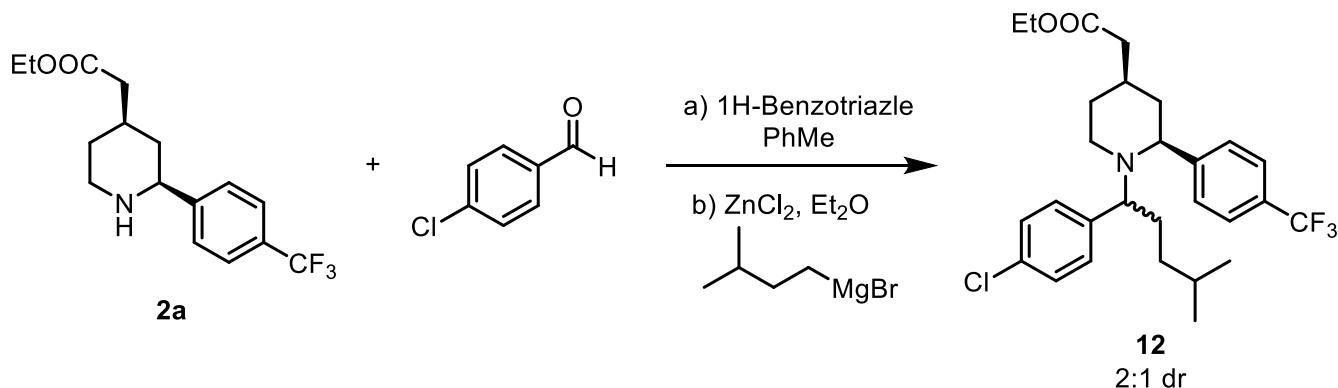

A mixture of $2 \mathbf{a}$ ( $240 \mathrm{mg}, 0.76 \mathrm{mmol}, 1.0$ equiv), 4-chlorobenzaldehyde (107 mg, 0.76 mmol, 1.0 equiv) and benzotriazole ( $90 \mathrm{mg}, 0.76 \mathrm{mmol}, 1.0$ equiv) in toluene ( $25 \mathrm{~mL}$ ) was stirred at reflux under $\mathrm{N}_{2}$ with a Dean-Stark trap in an oil bath for 18 hours. After cooling to RT, the solvent was removed in vacuo to give a thick oil, which was used without further purification.

To a solution of isopentylmagnesium bromide in diethyl ether $(1.0 \mathrm{M}, 2.28 \mathrm{~mL}, 3.0$ equiv) was added a solution of zinc chloride in diethyl ether $(1.0 \mathrm{M}, 2.28 \mathrm{~mL}, 3.0$ equiv) at $0{ }^{\circ} \mathrm{C}$. The cooling bath was removed and the reaction was stirred at RT for 1 hour. A solution of the benzotriazole adduct (from above) in dry DCM (2 mL) was added dropwise via cannula to the in situ generated organozinc solution at $0{ }^{\circ} \mathrm{C}$. After $45 \mathrm{~min}$ at $0{ }^{\circ} \mathrm{C}$, the mixture was stirred at RT for 18 hours. The reaction was quenched with saturated aqueous $\mathrm{NH}_{4} \mathrm{Cl}$. The mixture was diluted with DCM $(15 \mathrm{~mL})$ and $\mathrm{H}_{2} \mathrm{O}(15$ $\mathrm{mL})$, then filtered through Hyflo®. The layers were separated and the aqueous layer was extracted with DCM $(15 \mathrm{~mL} \times 3)$. The combined extracts were dried $\left(\mathrm{Na}_{2} \mathrm{SO}_{4}\right)$ and evaporated. The residue was purified by chromatography (silica, 3-6\% $\mathrm{Et}_{2} \mathrm{O} /$ isohexane) to give the product as an inseparable mixture $(271 \mathrm{mg}, 70 \%$ yield). 

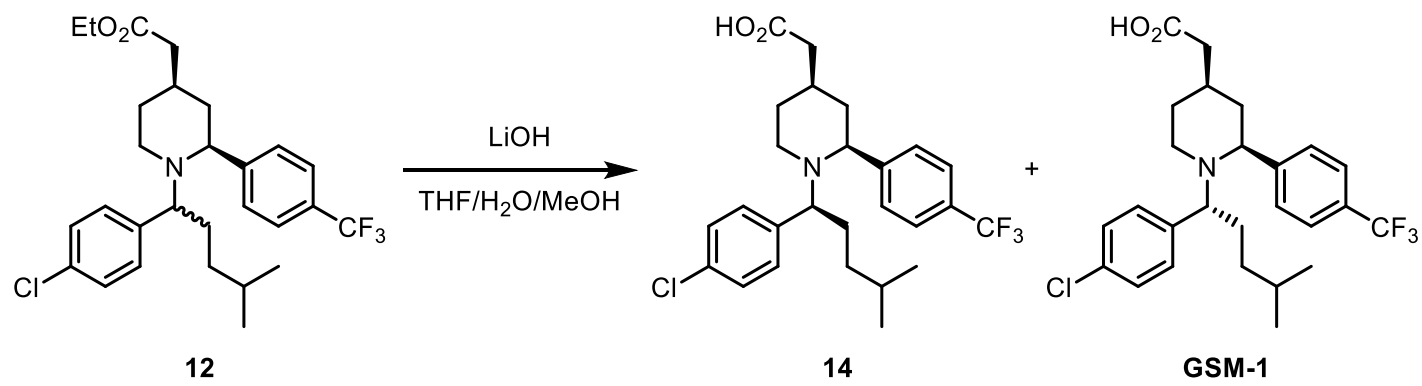

To a stirred mixture of $\mathbf{1 2}(0.1 \mathrm{mmol}, 1.0$ equiv, obtained in above step) in THF (1 $\mathrm{mL}) / \mathrm{H}_{2} \mathrm{O}(1 \mathrm{~mL}) / \mathrm{MeOH}(0.5 \mathrm{~mL})$ was added $\mathrm{LiOH}\left(1 \mathrm{mmol}, 10.0\right.$ equiv) at $0^{\circ} \mathrm{C}$. The reaction mixture was stirred at room temperature for 16 hours. The reaction mixture was acidified with $1 \mathrm{~N}$ hydrochloric acid to $\mathrm{pH}=7$. The mixture was extracted with ethyl acetate $(3 \mathrm{~mL} \times 3)$. The combined extracts were dried $\left(\mathrm{Na}_{2} \mathrm{SO}_{4}\right)$ and evaporated. The residue was purified by chromatography ( silica, ethyl acetate/petroleum ether $=1: 1$ ) to give the product. $^{[6]}$

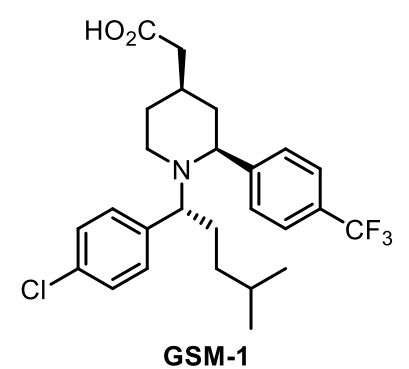

Yield 32mg, 65\%; Colorless oil; ${ }^{1} \mathrm{H}$ NMR $\left(400 \mathrm{MHz}\right.$, Methanol- $\left.d_{4}\right) \delta 7.75-7.62(\mathrm{~m}$, $4 \mathrm{H}), 7.29(\mathrm{~d}, J=8.2 \mathrm{~Hz}, 2 \mathrm{H}), 7.22(\mathrm{~d}, J=8.1 \mathrm{~Hz}, 2 \mathrm{H}), 3.95-3.84(\mathrm{~m}, 1 \mathrm{H}), 3.45(\mathrm{t}, J$ $=7.2 \mathrm{~Hz}, 1 \mathrm{H}), 2.83(\mathrm{dt}, J=11.9,3.4 \mathrm{~Hz}, 1 \mathrm{H}), 2.57-2.45(\mathrm{~m}, 1 \mathrm{H}), 2.16(\mathrm{~d}, J=7.4 \mathrm{~Hz}$, $2 \mathrm{H}), 1.99-1.88(\mathrm{~m}, 1 \mathrm{H}), 1.85(\mathrm{q}, J=7.5,7.1 \mathrm{~Hz}, 3 \mathrm{H}), 1.72(\mathrm{~d}, J=13.1 \mathrm{~Hz}, 1 \mathrm{H}), 1.50$ $-1.33(\mathrm{~m}, 2 \mathrm{H}), 1.26-1.04(\mathrm{~m}, 1 \mathrm{H}), 0.91-0.80(\mathrm{~m}, 1 \mathrm{H}), 0.83-0.75(\mathrm{~m}, 6 \mathrm{H}), 0.63-$ $0.49(\mathrm{~m}, 1 \mathrm{H}) .{ }^{13} \mathrm{C}\left\{{ }^{1} \mathrm{H}\right\}$ NMR (100 MHz, Methanol- $\left.d_{4}\right) \delta 175.3,147.8,138.9,132.6$, $130.4,129.6$ (q, $J=32.3 \mathrm{~Hz}), 128.2,127.8,125.5$ (q, $J=3.7 \mathrm{~Hz}), 124.3$ (q, $J=271.0$ $\mathrm{Hz}), 64.9,62.0,44.7,41.9,40.8,36.1,33.4,31.5,27.9,21.7,21.4,19.7 .{ }^{19} \mathrm{~F}$ NMR $(376$ $\mathrm{MHz}$, Methanol- $\left.d_{4}\right) \delta$-63.8. HRMS (ESI): calcd for $\mathrm{C}_{26} \mathrm{H}_{32} \mathrm{ClF}_{3} \mathrm{NO}_{2}[\mathrm{M}+\mathrm{H}]^{+}$: 482.2074 , found 482.2077 .<smiles>CC(C)CCC(c1ccc(Cl)cc1)N1CCC(CC(=O)O)CC1c1ccc(C(F)(F)F)cc1</smiles>

Yield 16mg, 32\%; Colorless oil; ${ }^{1} \mathrm{H}$ NMR (400 MHz, Methanol- $\left.d_{4}\right) \delta 7.70(\mathrm{~d}, J=8.0$ $\mathrm{Hz}, 2 \mathrm{H}), 7.59$ (d, $J=7.9 \mathrm{~Hz}, 2 \mathrm{H}), 7.30(\mathrm{~d}, J=8.0 \mathrm{~Hz}, 2 \mathrm{H}), 6.95(\mathrm{~d}, J=8.0 \mathrm{~Hz}, 2 \mathrm{H})$, $3.60(\mathrm{t}, J=7.7 \mathrm{~Hz}, 1 \mathrm{H}), 3.29-3.23(\mathrm{~m}, 1 \mathrm{H}), 3.20(\mathrm{dd}, J=11.2,2.8 \mathrm{~Hz}, 1 \mathrm{H}), 2.22-$ 
$2.05(\mathrm{~m}, 2 \mathrm{H}), 2.03-1.94(\mathrm{~m}, 1 \mathrm{H}), 1.88(\mathrm{dd}, J=11.0,6.4 \mathrm{~Hz}, 1 \mathrm{H}), 1.81(\mathrm{td}, J=9.7,4.8$ $\mathrm{Hz}, 2 \mathrm{H}), 1.76-1.69(\mathrm{~m}, 1 \mathrm{H}), 1.69-1.57(\mathrm{~m}, 1 \mathrm{H}), 1.38$ (tt, $J=11.7,5.3 \mathrm{~Hz}, 2 \mathrm{H}), 1.29$ $-1.19(\mathrm{~m}, 1 \mathrm{H}), 1.19-1.06(\mathrm{~m}, 1 \mathrm{H}), 0.98-0.86(\mathrm{~m}, 1 \mathrm{H}), 0.81(\mathrm{dd}, J=6.8,2.7 \mathrm{~Hz}, 6 \mathrm{H})$. ${ }^{13} \mathrm{C}\left\{{ }^{1} \mathrm{H}\right\}$ NMR (100 MHz, Methanol- $\left.d_{4}\right) \delta 175.0,149.1,135.0,132.7,130.6,129.1$ (q, $J=32.0 \mathrm{~Hz}), 128.2,127.5,125.3(\mathrm{q}, J=3.7 \mathrm{~Hz}), 124.3(\mathrm{q}, J=270.8 \mathrm{~Hz}), 64.8,62.0$, 45.3, 43.4, 40.5, 35.6, 33.3, 31.8, 29.5, 27.6, 21.7, 21.4. ${ }^{19} \mathrm{~F}$ NMR (376 MHz, MeOD) $\delta$ -63.8. HRMS (ESI): calcd for $\mathrm{C}_{26} \mathrm{H}_{32} \mathrm{ClF}_{3} \mathrm{NO}_{2}[\mathrm{M}+\mathrm{H}]^{+}:$482.2074, found 482.2077.

\section{References:}

[1] K. Murai, J. Nakajima, A. Nakamura, N. Hyogo, H. Fujioka. Chem. Asian J. 2014, 9, 3511.

[2] V. M. Foley, C. M. McSweeney, K. S. Eccles, S. E. Lawrence, G. P. McGlacken. Org. Lett. 2015, 17, 5642.

[3] J. Tanuwidjaja, H. M. Peltier, J. A. Ellman. J. Org. Chem. 2007, 72, 626; L. R. Reddy,

S. G. Das, Y. Liu, M. Prashad. J. Org. Chem. 2010, 75, 2236.

[4] K. V. Emelen, T. D. Wit, G. J. Hoornaert, F. Compernolle. Tetrahedron, 2002, 58, 4225.

[5] A. Hall, R. L. Elliott, G. M. P. Giblin, I. Hussain, J. Musgrave, A. Naylor, R. Sasse, B. Smith. Bioorg. Med. Chem. Lett, 2010, 20, 1306.

[6] A. Rennhack, T. Jumpertz, J. Ness, S. Baches, C. U. Pietrzik, S. Weggen, B. Bulic. Bioorg. Med. Chem. 2012, 20, 6523.

\section{Crystallographic data for the opposite enantiomer of 6a}

Single crystals of $\mathbf{6 a}^{\prime}$ were grown in dichloromethane and hexane. DCM $(0.5 \mathrm{~mL})$ was added to $6 \mathbf{a}^{\prime}$ ( $30 \mathrm{mg}$ in a $1.5 \mathrm{~mL}$ vial) followed by $0.5 \mathrm{~mL}$ of hexane. The $1.5 \mathrm{~mL}$ vial was capped and placed at room temperature in the experimental cabinet for 3 days, whereupon crystals formed. A clear light colorless rod shaped crystal of 6a' was used for the X-ray crystallographic analysis. The X-ray intensity data were measured at 167 $\mathrm{K}$, on an AtlasS2 diffractometer. The crystal data of $\mathbf{6} \mathbf{a}^{\prime}$ have been deposited in CCDC and have been displayed at 50\% ellipsoid contour probability level.

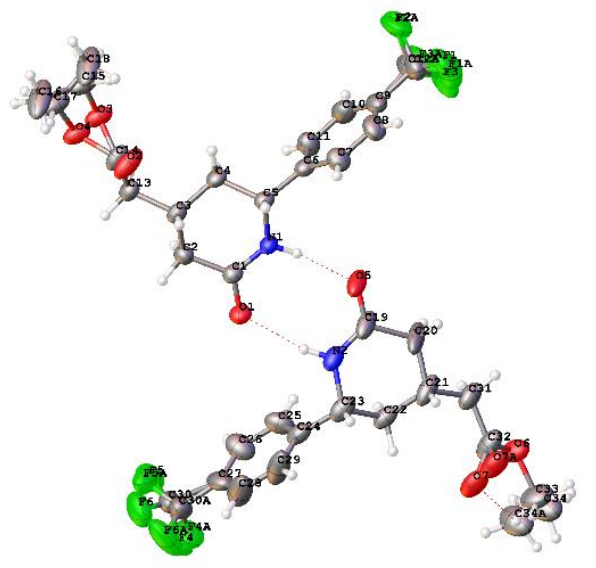




\begin{tabular}{|c|c|}
\hline Parameter & Value \\
\hline Empirical formula & $\mathrm{C}_{16} \mathrm{H}_{18} \mathrm{~F}_{3} \mathrm{NO}_{3}$ \\
\hline Formula weight & 329.31 \\
\hline Temperature / K & $116.60(14)$ \\
\hline Crystal system & monoclinic \\
\hline Space group & $\mathrm{P} 21$ \\
\hline $\mathrm{a} / \AA, \mathrm{b} / \AA, \mathrm{c} / \AA$ & $5.4922(2), 11.1842(6), 25.9330(14)$ \\
\hline$a /{ }^{\circ}, \beta /{ }^{\circ}, \gamma /{ }^{\circ}$ & $90.00,94.052(4), 90.00$ \\
\hline Volume / $\AA^{3}$ & $1588.97(14)$ \\
\hline $\mathrm{Z}$ & 4 \\
\hline$\rho_{\text {calc }} / \mathrm{mg} \mathrm{mm}^{-3}$ & 1.377 \\
\hline$\mu / \mathrm{mm}^{-1}$ & 1.014 \\
\hline $\mathrm{F}(000)$ & 688 \\
\hline Crystal size $/ \mathrm{mm}^{3}$ & $0.43 \times 0.08 \times 0.08$ \\
\hline $2 \theta$ range for data collection $(2 \theta)$ & 8.62 to $142.46^{\circ}$ \\
\hline Index ranges & $-6 \leq \mathrm{h} \leq 4,-13 \leq \mathrm{k} \leq 13,-30 \leq 1 \leq 31$ \\
\hline Reflections collected & 10597 \\
\hline Independent reflections & $5985[\mathrm{R}(\mathrm{int})=0.0325($ inf- $0.9 \AA)]$ \\
\hline Data/restraints/parameters & $5985 / 279 / 511$ \\
\hline Goodness-of-fit on $\mathrm{F}^{2}$ & 1.024 \\
\hline Final R indexes $\left[\mathrm{I}>2 \sigma(\mathrm{I})\right.$ i.e. $\left.\mathrm{F}_{\mathrm{o}}>4 \sigma\left(\mathrm{F}_{\mathrm{o}}\right)\right]$ & $\mathrm{R}_{1}=0.0589, \mathrm{wR}_{2}=0.1388$ \\
\hline Final $\mathrm{R}$ indexes [all data] & $\mathrm{R}_{1}=0.0663, \mathrm{wR}_{2}=0.1461$ \\
\hline Largest diff. peak/hole / e $\AA^{-3}$ & $0.302 /-0.257$ \\
\hline Flack Parameters & $0.3(2)$ \\
\hline Completeness & 0.9993 \\
\hline
\end{tabular}

Crystal Data for ent-6a . $\mathrm{C}_{16} \mathrm{H}_{18} \mathrm{~F}_{3} \mathrm{NO}_{3}, M=329.31$, monoclinic, $a=$ 5.4922(2) $\AA, b=11.1842(6) \AA, c=25.9330(14) \AA, \beta=94.052(4)^{\circ}, U=$ 1588.97(14) $\AA^{3}, T=116.60(14)$, space group $\mathrm{P} 2{ }_{1}$ (no. 4), $Z=4, \mu(\mathrm{Cu} \mathrm{K} \alpha)=1.014$, 10597 reflections measured, 5985 unique $\left(R_{\text {int }}=0.0325\right)$ which were used in all calculations. The final $w R\left(F_{2}\right)$ was 0.1461 (all data). 


\section{NMR Spectra}

${ }^{1} \mathrm{H}$ NMR of 13a $\left(400 \mathrm{MHz}, \mathrm{CDCl}_{3}\right)$

$\underbrace{0}_{0}$<smiles>CCOCC(CC(=O)OCC)C(C(=O)OCC)C(=O)c1ccc(C(F)(F)F)cc1</smiles>

$13 a$

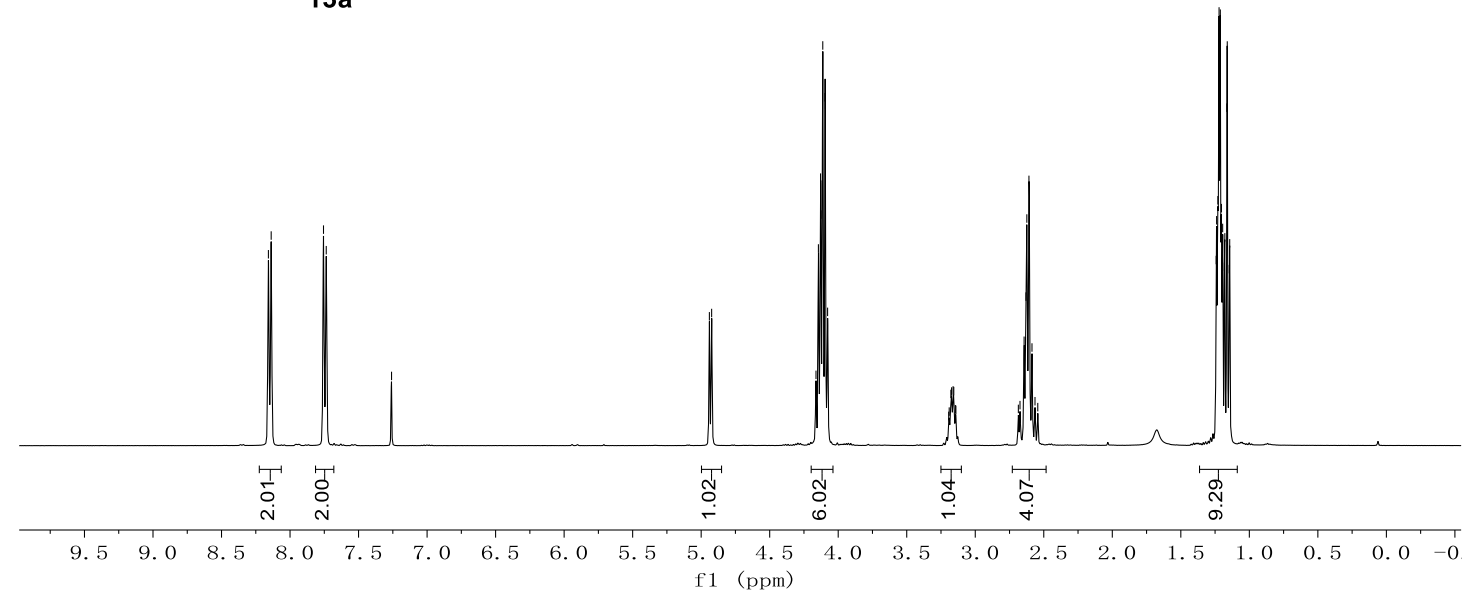

${ }^{13} \mathrm{C}\left\{{ }^{1} \mathrm{H}\right\}$ NMR of 13a $\left(100 \mathrm{MHz}, \mathrm{CDCl}_{3}\right)$

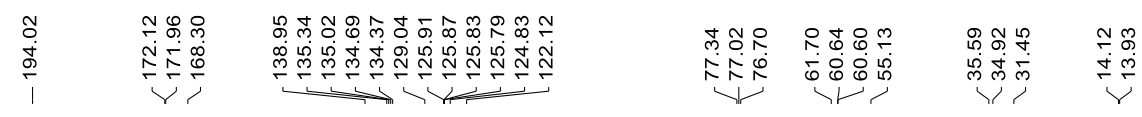<smiles>CCOCC(CC(=O)OCC)C(C(=O)OCC)C(=O)c1ccc(C(F)(F)F)cc1</smiles>

$13 a$

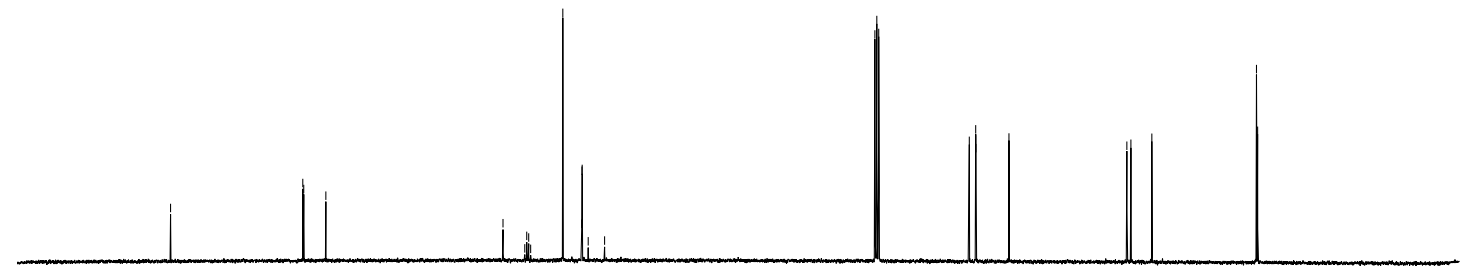

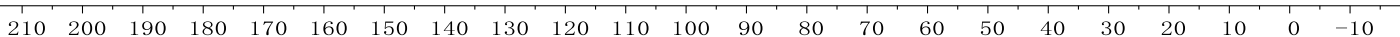
f1 (ppm) 
${ }^{19} \mathrm{~F}$ NMR of 13a $\left(376 \mathrm{MHz}, \mathrm{CDCl}_{3}\right)$

Nָ<smiles>CCOCC(CC(=O)OCC)C(OCC)C(=O)c1ccc(C(F)(F)F)cc1</smiles>

$13 a$

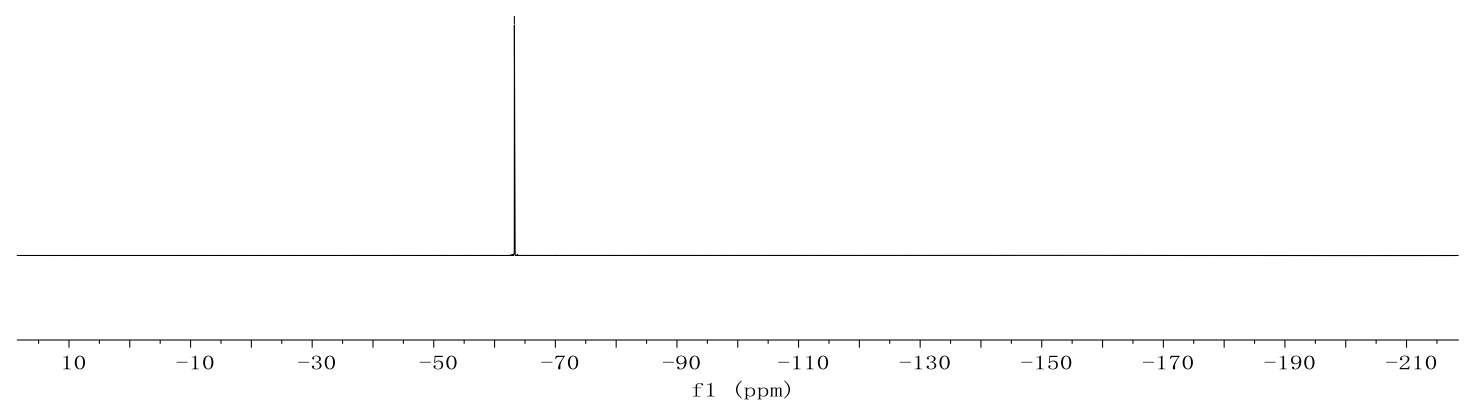


${ }^{1} \mathrm{H}$ NMR of $\mathbf{4 a}\left(400 \mathrm{MHz}, \mathrm{CDCl}_{3}\right)$

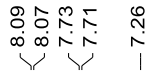

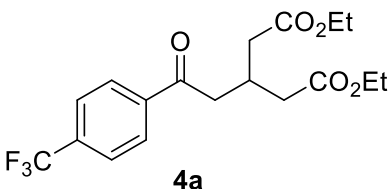

$4 a$
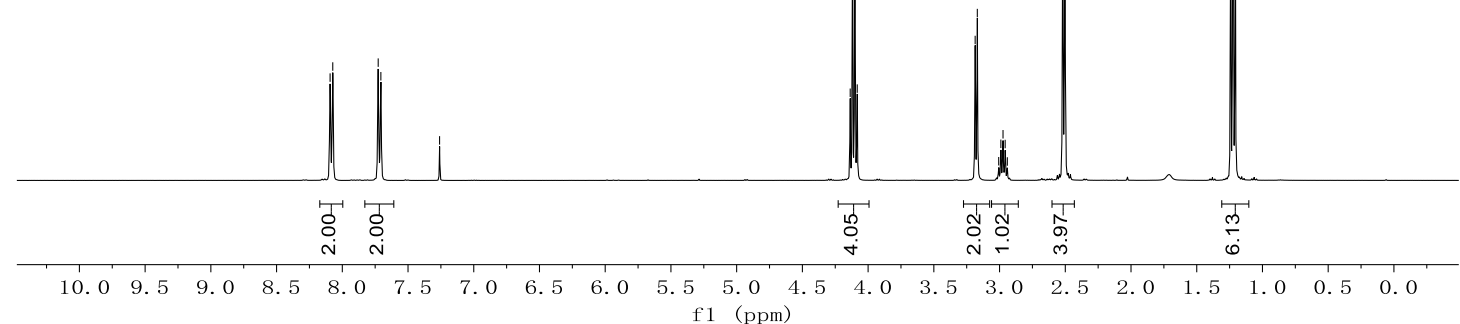

${ }^{13} \mathrm{C}\left\{{ }^{1} \mathrm{H}\right\}$ NMR of $\mathbf{4 a}\left(100 \mathrm{MHz}, \mathrm{CDCl}_{3}\right)$

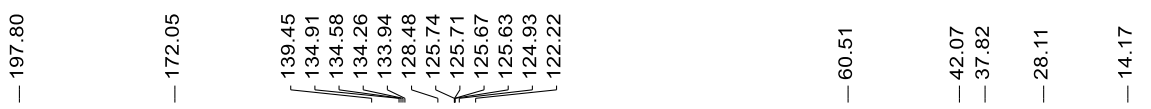

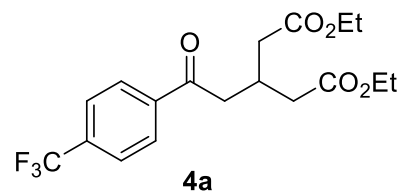

$4 a$

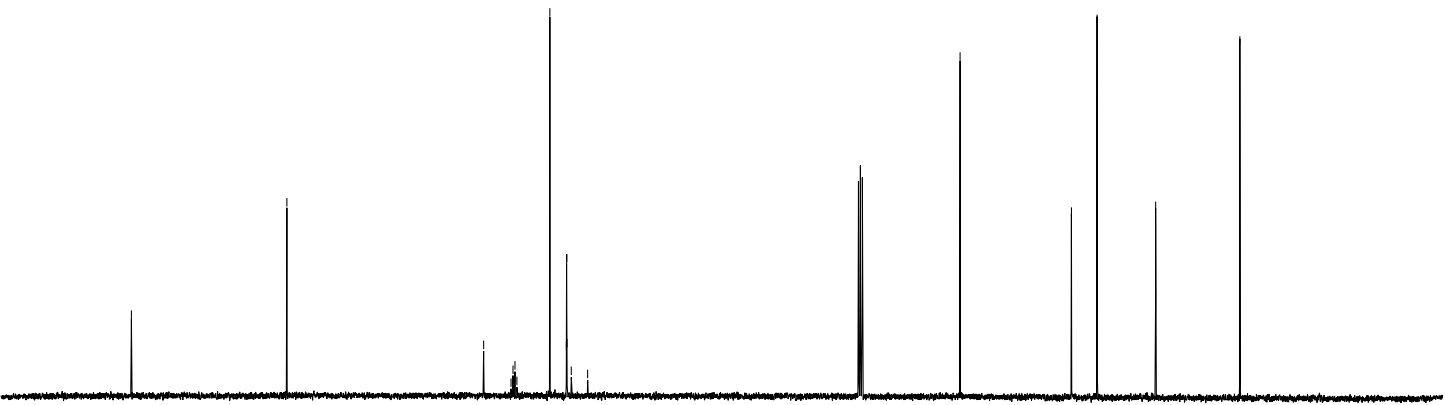

$\begin{array}{lllllllllllllllllllllll}210 & 200 & 190 & 180 & 170 & 160 & 150 & 140 & 130 & 120 & 110 & 100 & 90 & 80 & 70 & 60 & 50 & 40 & 30 & 20 & 10 & 0 & -10\end{array}$ f1 (ppm) 
${ }^{19} \mathrm{~F}$ NMR of $4 \mathbf{a}\left(376 \mathrm{MHz}, \mathrm{CDCl}_{3}\right)$
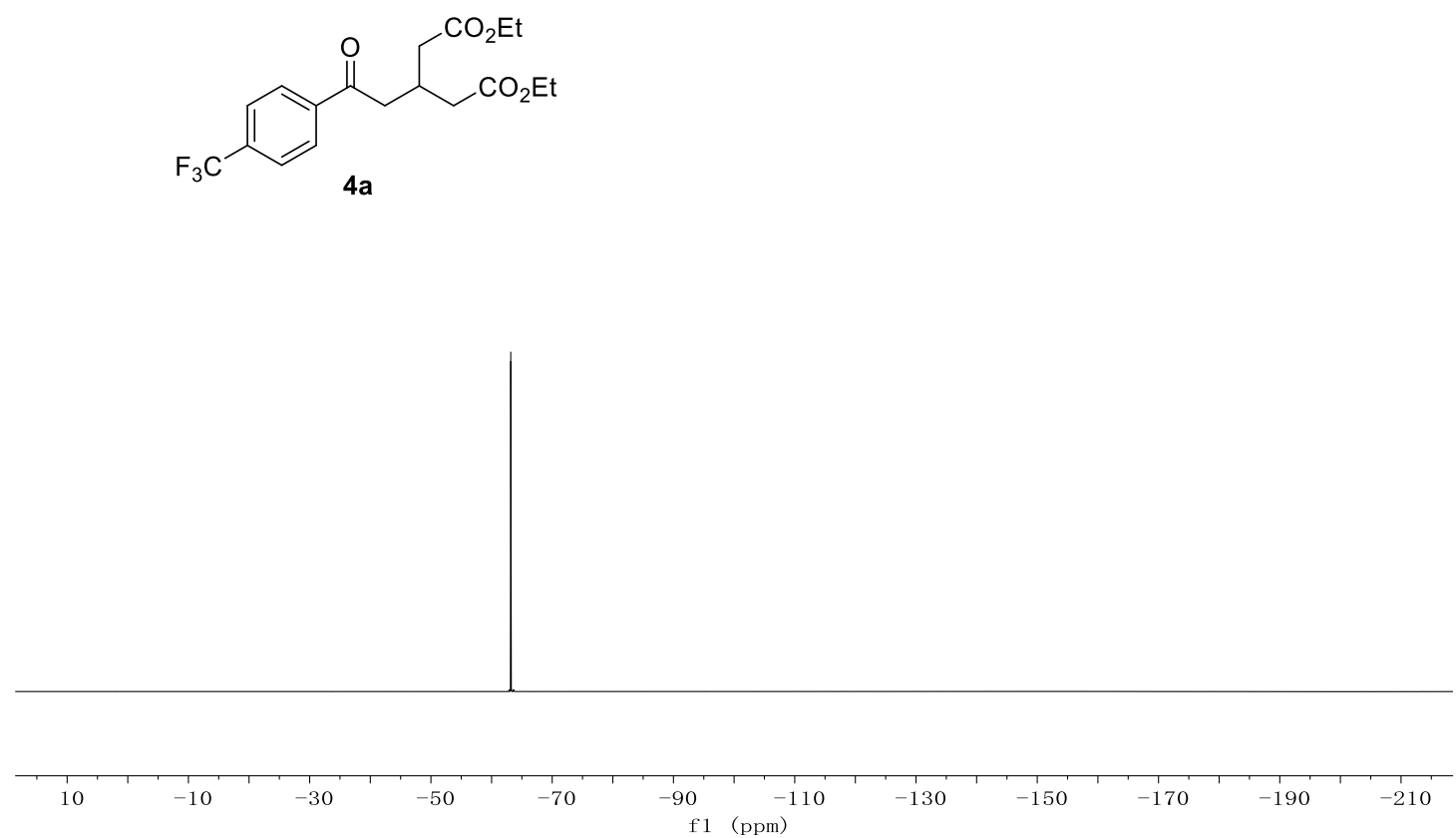
${ }^{1} \mathrm{H}$ NMR of $7 \mathbf{a}\left(400 \mathrm{MHz}, \mathrm{CDCl}_{3}\right)$

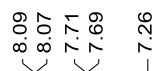

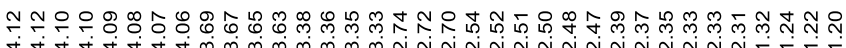<smiles>CCOCC(CC(=O)OCC)CC(=NS(=O)C(C)(C)C)c1ccc(C(F)(F)F)cc1</smiles>

$7 \mathbf{a}$

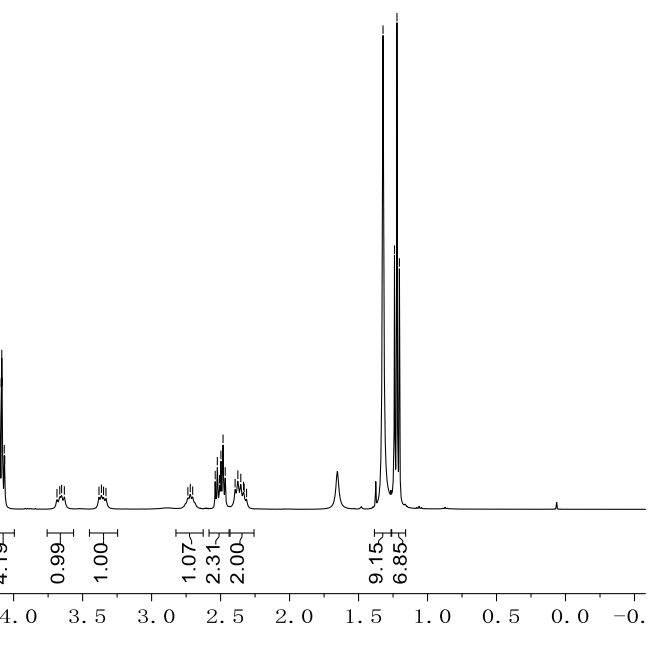

${ }^{13} \mathrm{C}\left\{{ }^{1} \mathrm{H}\right\}$ NMR of $7 \mathbf{a}\left(100 \mathrm{MHz}, \mathrm{CDCl}_{3}\right)$

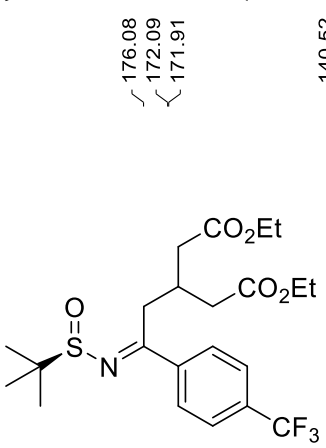

$7 a$

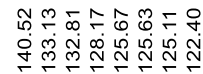

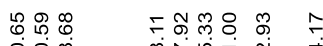

نํำ

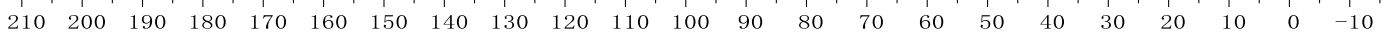
f1 (ppm) 
${ }^{19} \mathrm{~F}$ NMR of $7 \mathbf{a}\left(376 \mathrm{MHz}, \mathrm{CDCl}_{3}\right)$
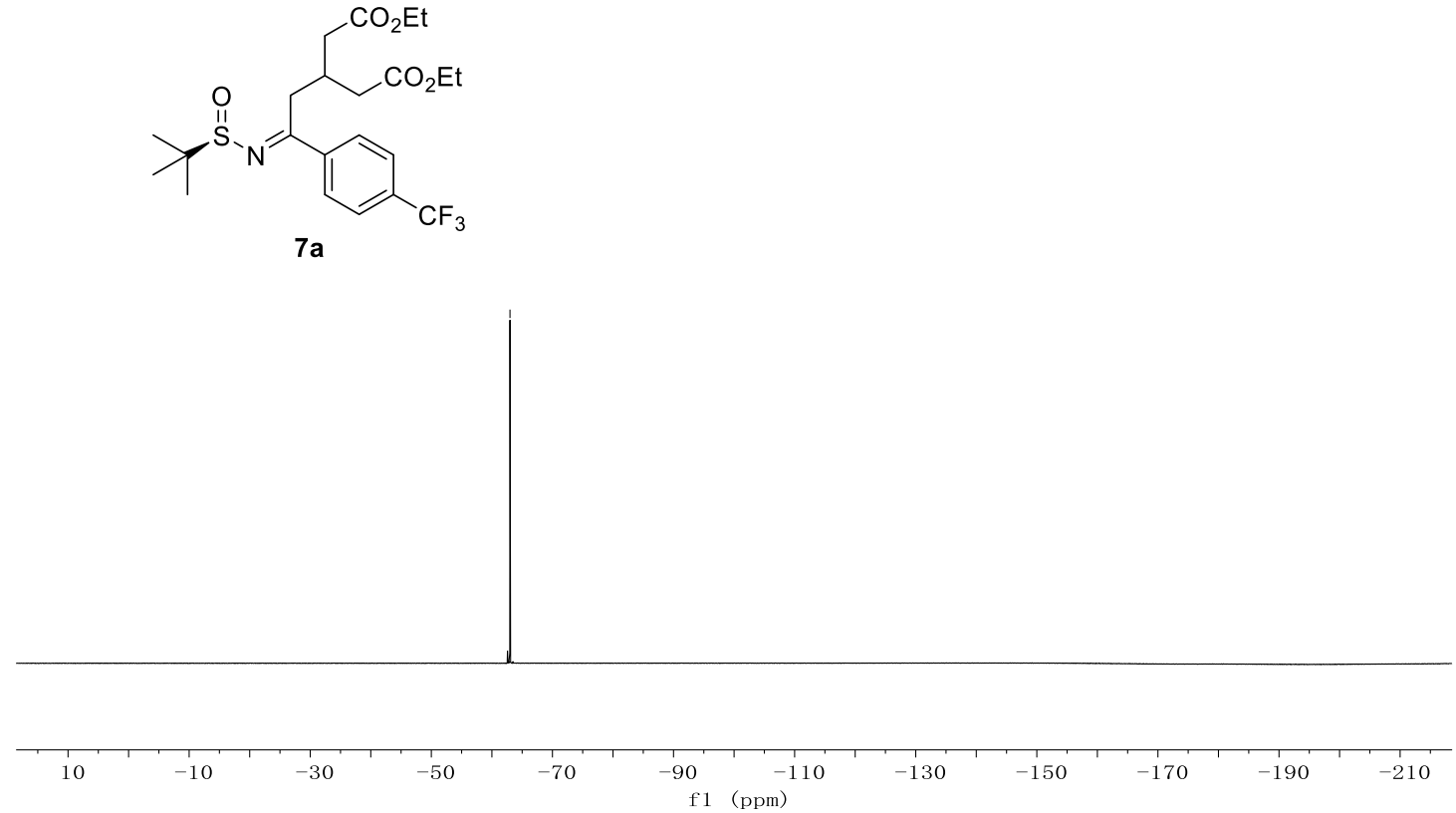
${ }^{1} \mathrm{H}$ NMR of $\mathbf{8 a}\left(400 \mathrm{MHz}, \mathrm{CDCl}_{3}\right)$

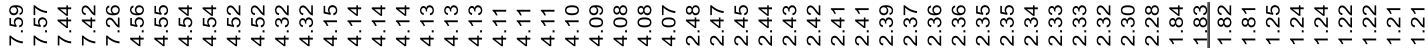
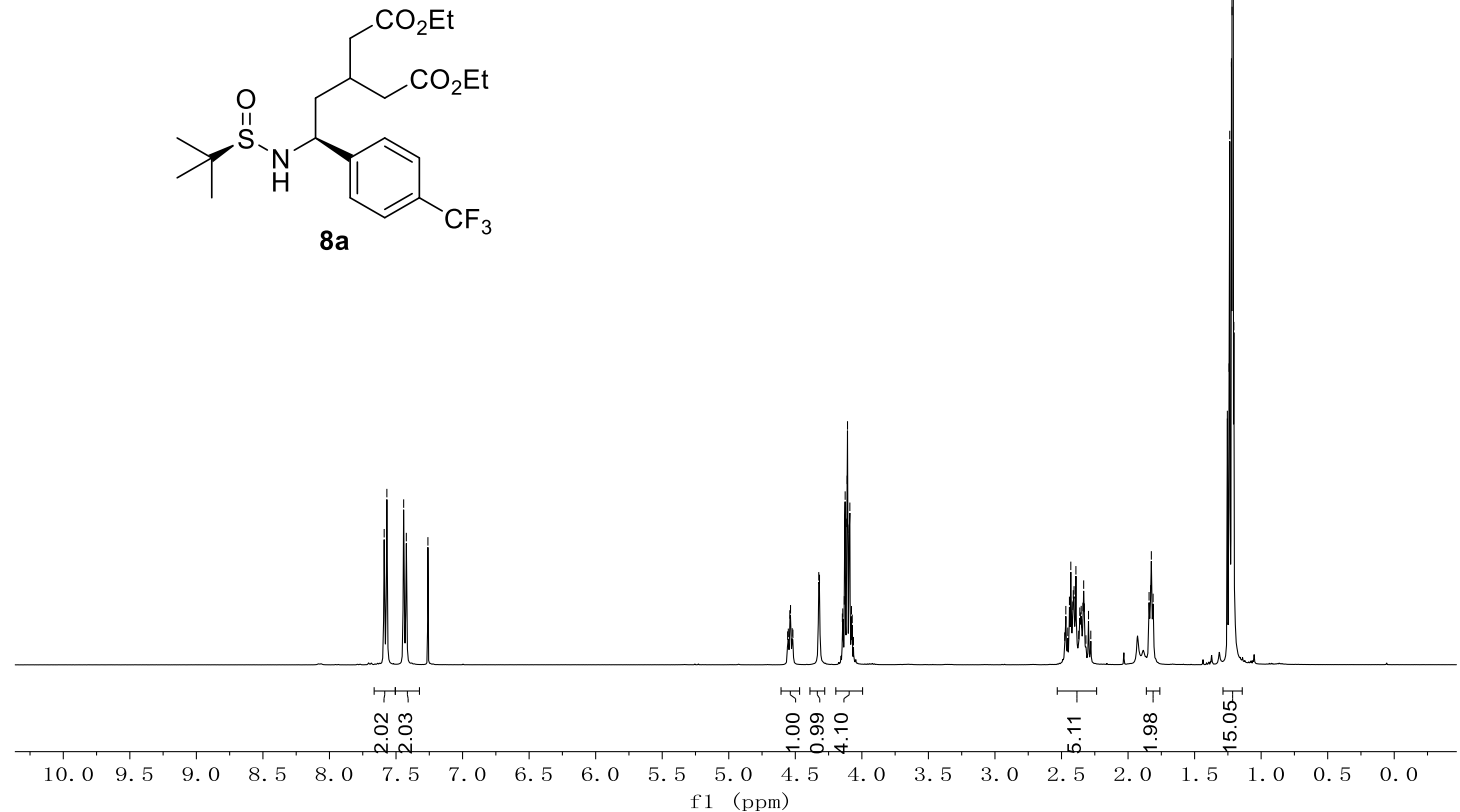

Crude ${ }^{1} \mathrm{H}$ NMR of $8 \mathbf{a}\left(400 \mathrm{MHz}, \mathrm{CDCl}_{3}\right)$

cp-13-30-201007. 10. fid

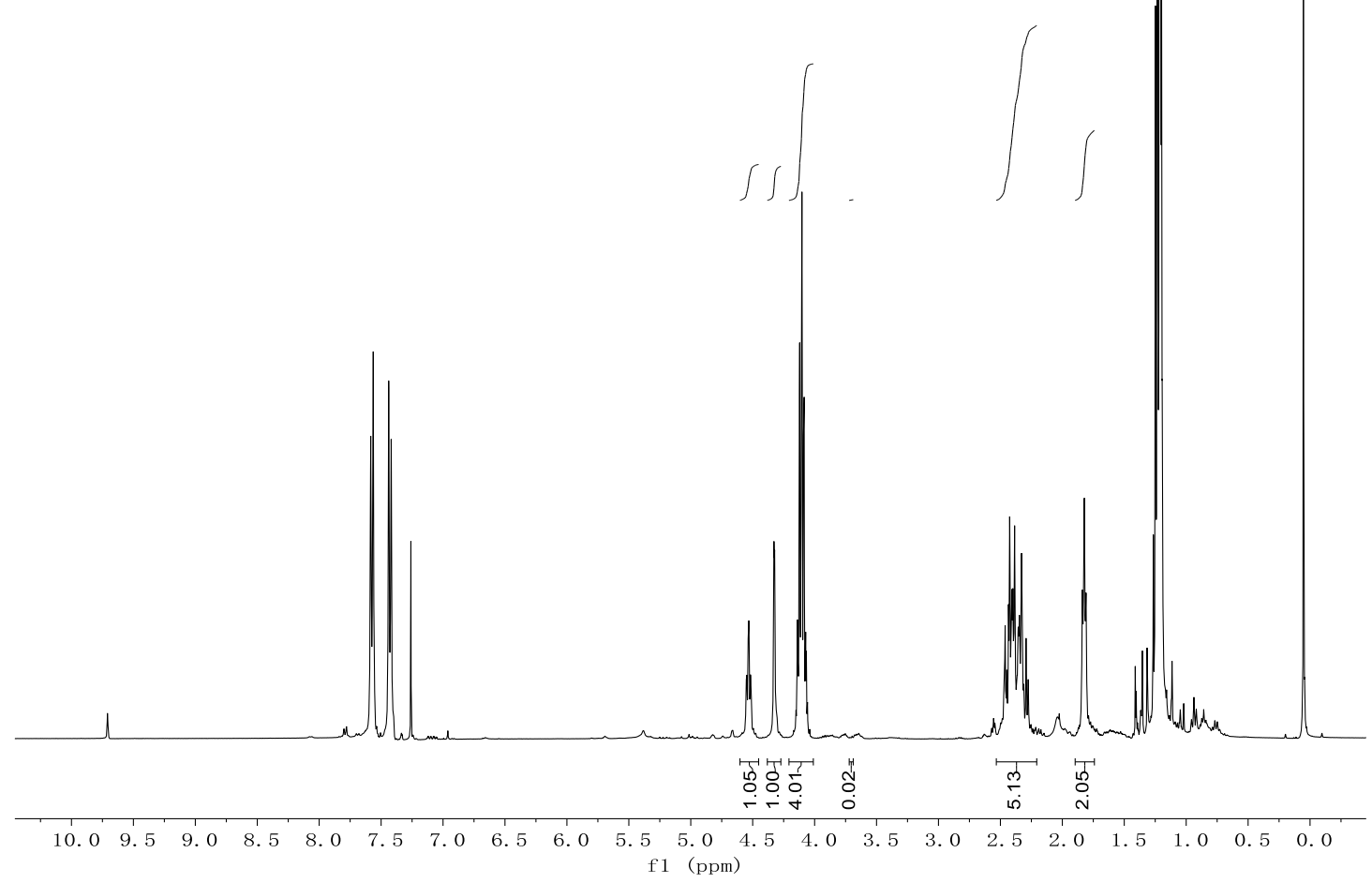


${ }^{13} \mathrm{C}\left\{{ }^{1} \mathrm{H}\right\} \mathrm{NMR}$ of $\mathbf{8 a}\left(100 \mathrm{MHz}, \mathrm{CDCl}_{3}\right)$

인

竞

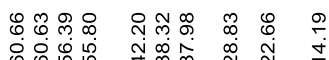

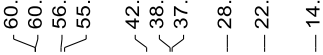

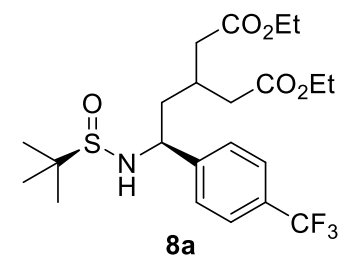

8a

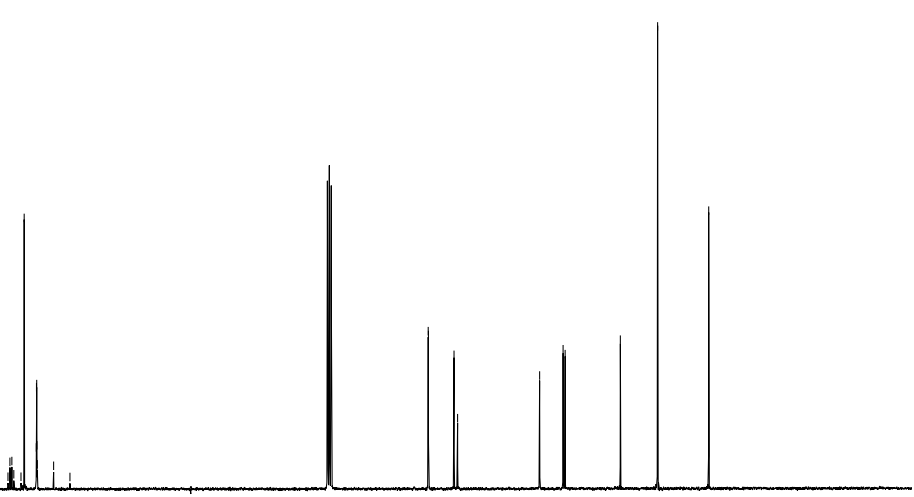

$\begin{array}{llllllllllllllllllllllll}210 & 200 & 190 & 180 & 170 & 160 & 150 & 140 & 130 & 120 & 110 & 100 & 90 & 80 & 70 & 60 & 50 & 40 & 30 & 20 & 10 & 0 & -10\end{array}$ f1 (ppm)

${ }^{19} \mathrm{~F}$ NMR of $\mathbf{8 a}\left(376 \mathrm{MHz}, \mathrm{CDCl}_{3}\right)$

㐫<smiles>CCOCCC(CC(=O)OCC)CC(NS(=O)C(C)(C)C)c1ccc(C(F)(F)F)cc1</smiles>

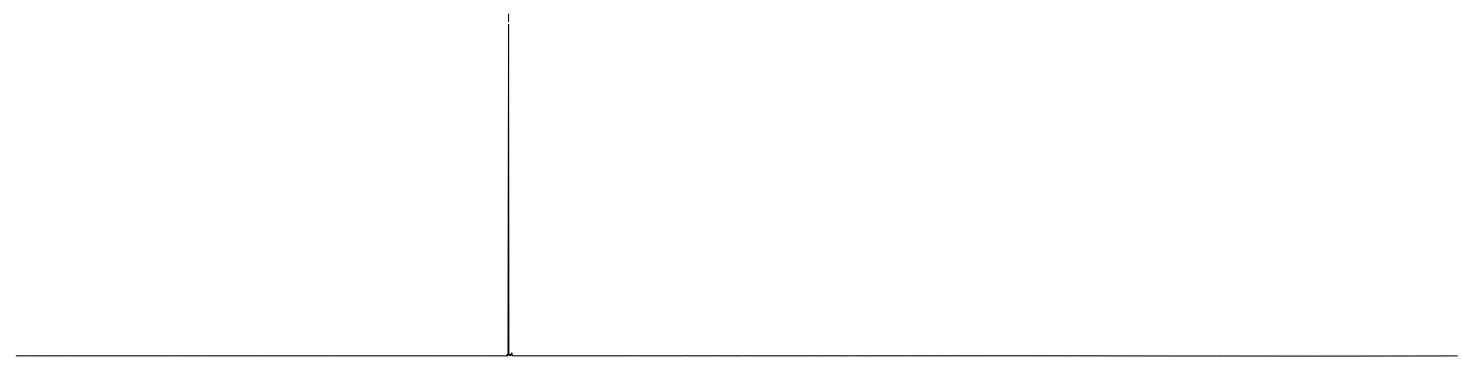

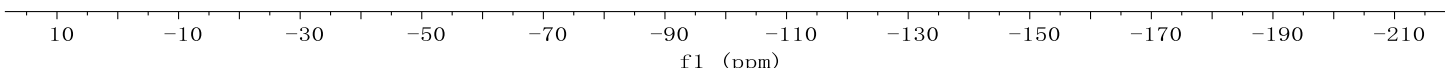


<smiles>CCOC(=O)CC(CC(=O)OCC)C[C@H](NS(=O)C(C)(C)C)c1ccc(C(F)(F)F)cc1</smiles>

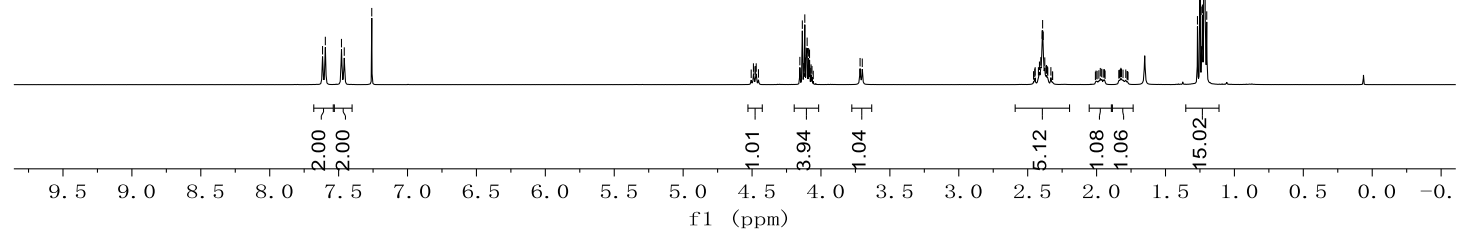

${ }^{13} \mathrm{C}\left\{{ }^{1} \mathrm{H}\right\} \mathrm{NMR}$ of $\mathbf{8} \mathbf{a}^{\prime}\left(100 \mathrm{MHz}, \mathrm{CDCl}_{3}\right)$

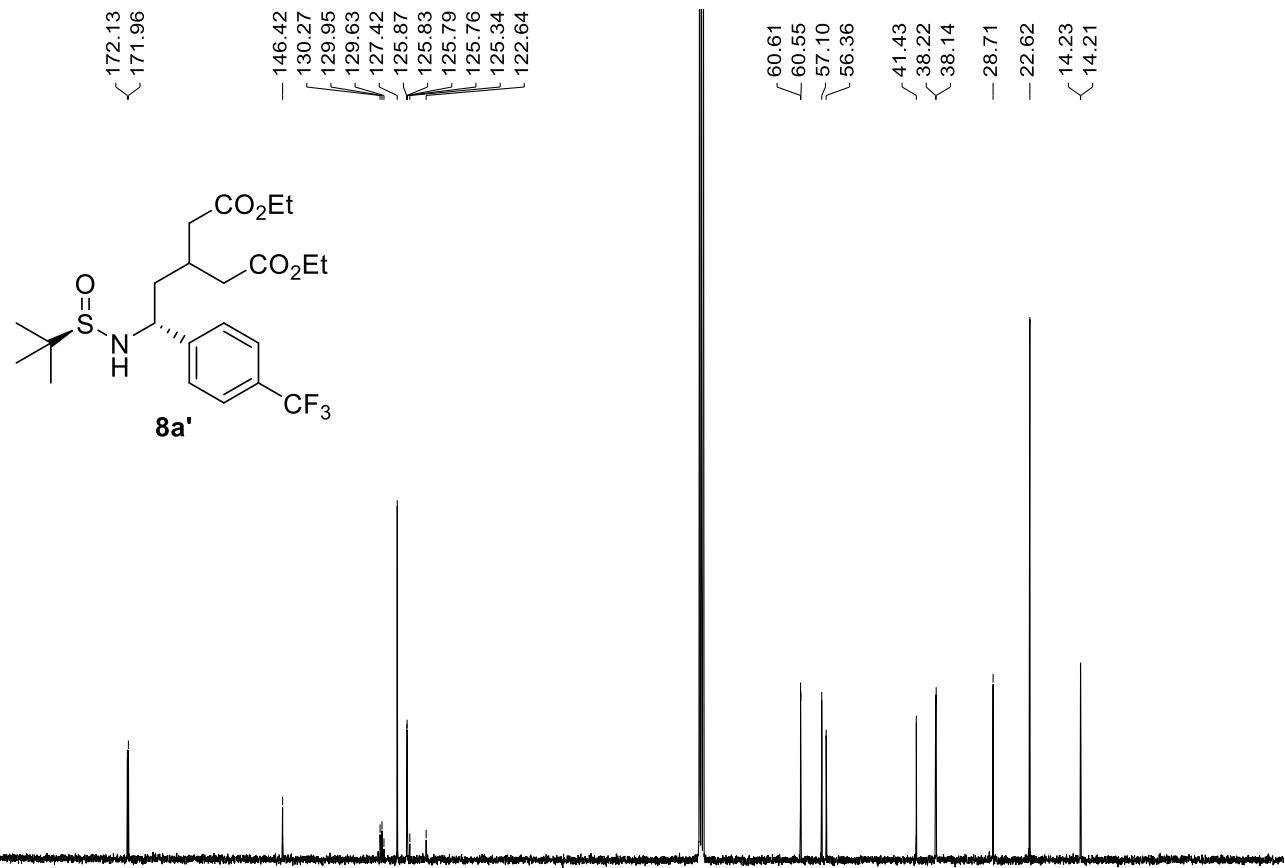

$\begin{array}{llllllllllllllllllllllll}210 & 200 & 190 & 180 & 170 & 160 & 150 & 140 & 130 & 120 & 110 & 100 & 90 & 80 & 70 & 60 & 50 & 40 & 30 & 20 & 10 & 0 & -10\end{array}$ f1 (ppm) 
${ }^{19} \mathrm{~F}$ NMR of $\mathbf{8 a}^{\prime}\left(376 \mathrm{MHz}, \mathrm{CDCl}_{3}\right)$

$\stackrel{\infty}{\stackrel{\infty}{\leftrightarrow}}$
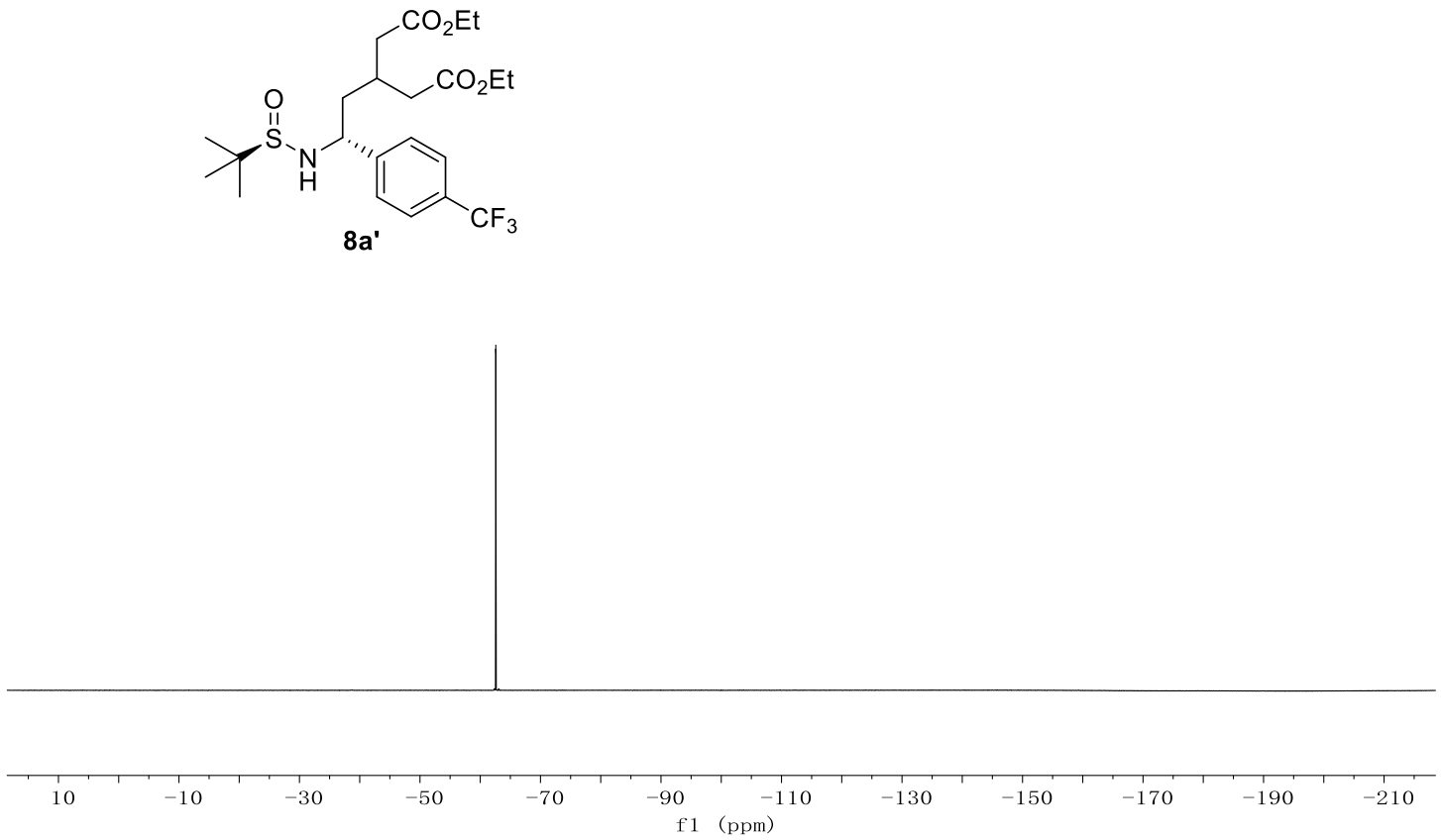
${ }^{1} \mathrm{H}$ NMR of $6 \mathbf{a}\left(400 \mathrm{MHz}, \mathrm{CDCl}_{3}\right)$

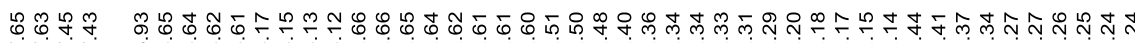

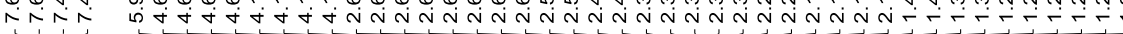<smiles>CCOC(=O)C[C@@H]1CC(=O)NC(c2ccc(C(F)(F)F)cc2)C1</smiles>

$6 a$

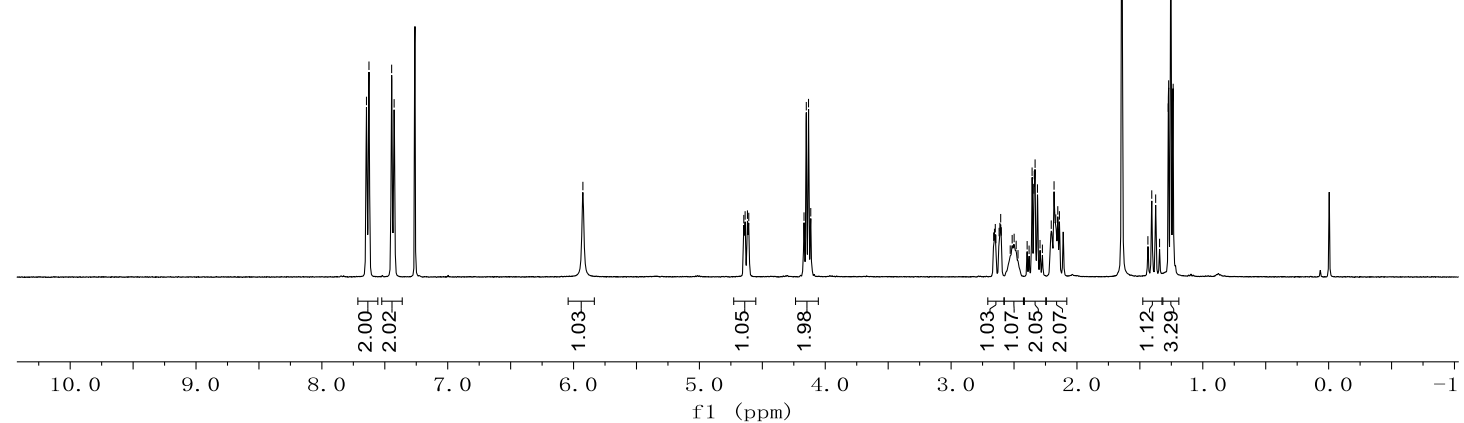

${ }^{13} \mathrm{C}\left\{{ }^{1} \mathrm{H}\right\}$ NMR of $\mathbf{6 a}\left(100 \mathrm{MHz}, \mathrm{CDCl}_{3}\right)$

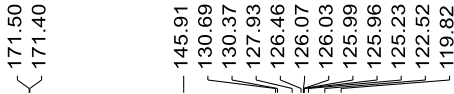

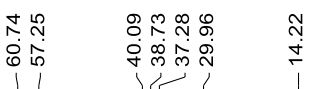

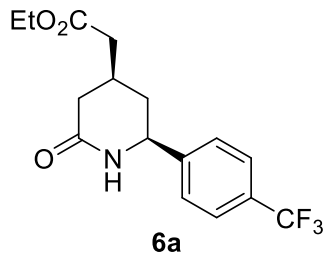

6a

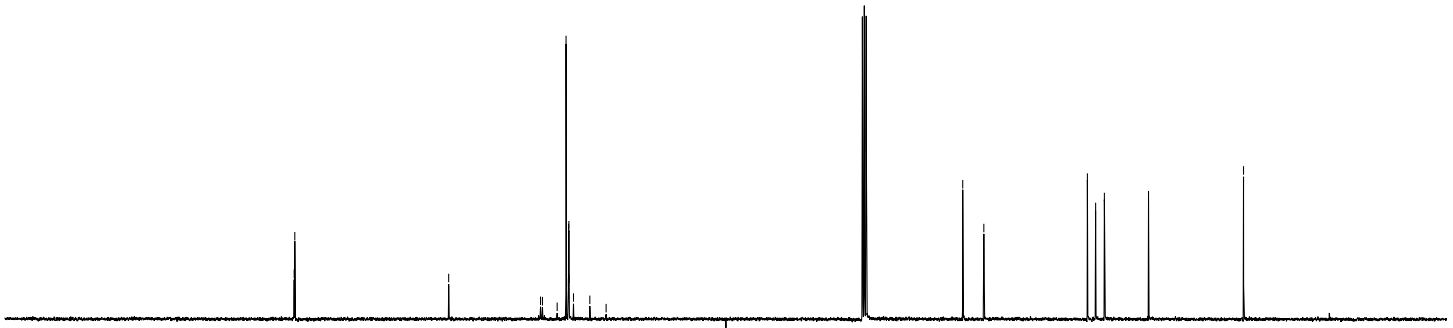

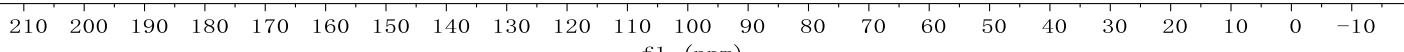
f1 (ppm) 
${ }^{19} \mathrm{~F}$ NMR of $\mathbf{6 a}\left(376 \mathrm{MHz}, \mathrm{CDCl}_{3}\right)$

品
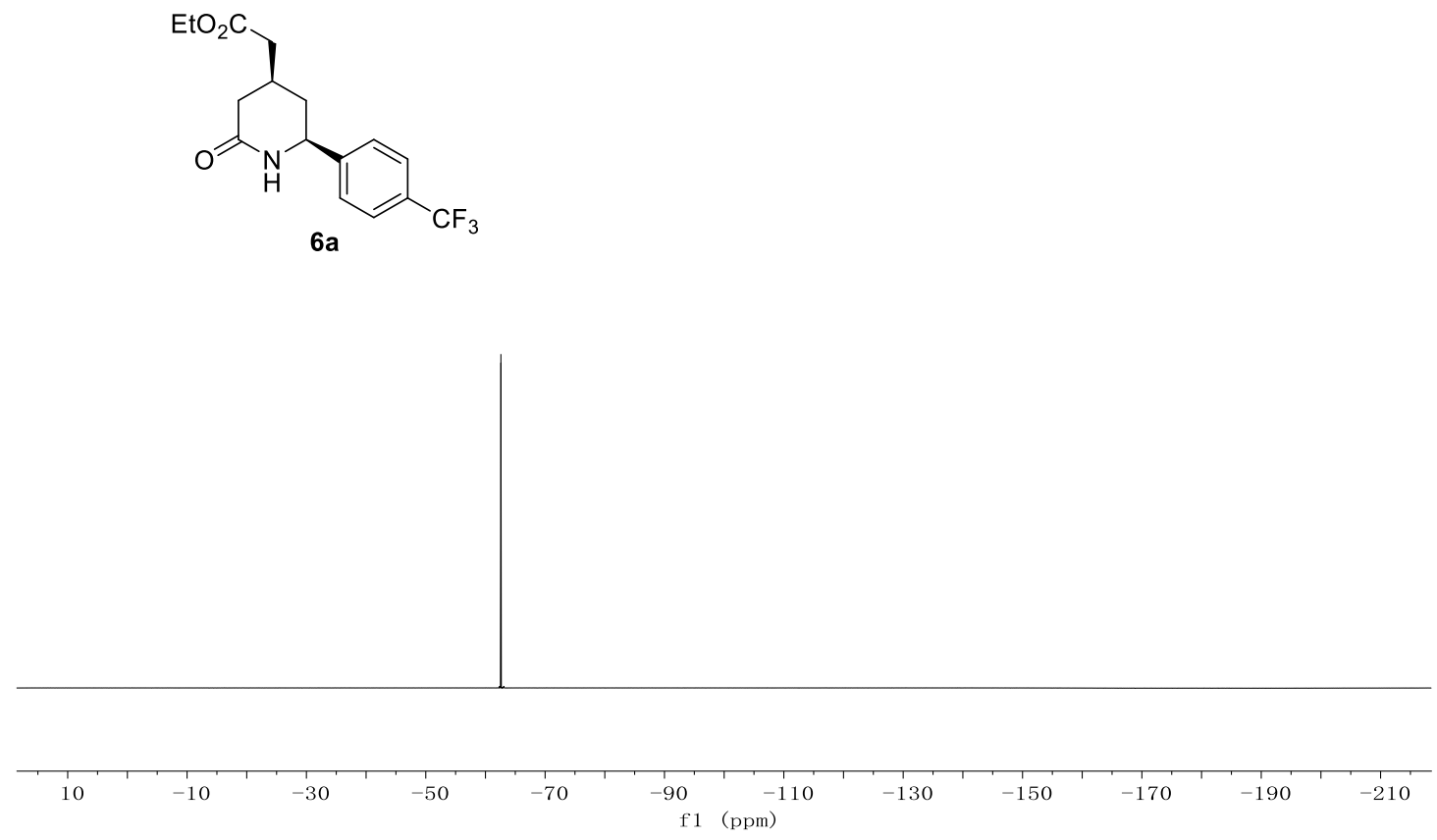
${ }^{1} \mathrm{H}$ NMR of $2 \mathbf{a}\left(400 \mathrm{MHz}, \mathrm{CDCl}_{3}\right)$

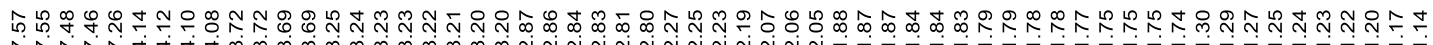<smiles>CCOC(=O)CC1CCNC(c2ccc(C(F)(F)F)cc2)C1</smiles>

2a

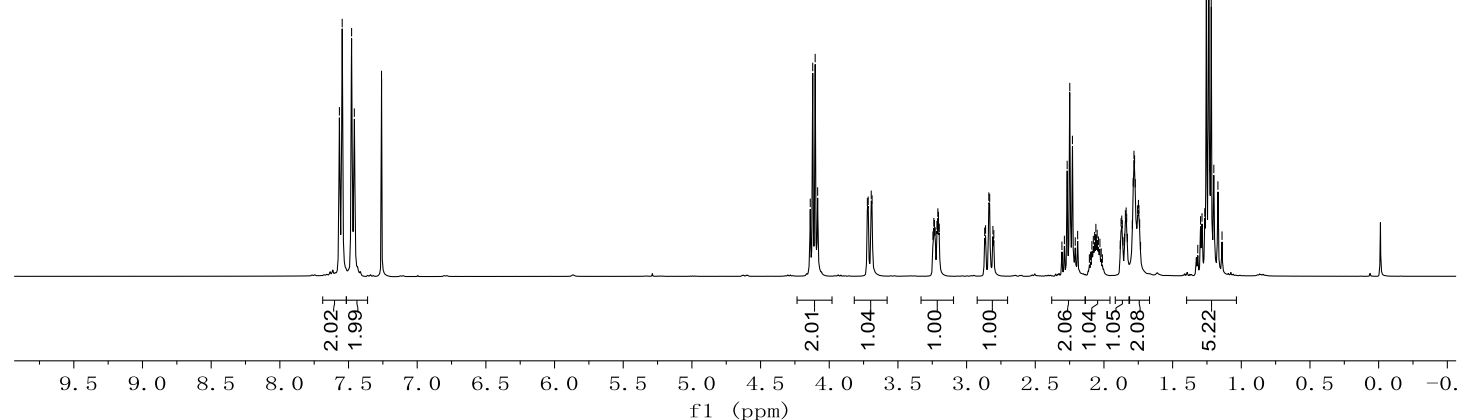

${ }^{13} \mathrm{C}\left\{{ }^{1} \mathrm{H}\right\} \mathrm{NMR}$ of $\mathbf{2 a}\left(100 \mathrm{MHz}, \mathrm{CDCl}_{3}\right)$

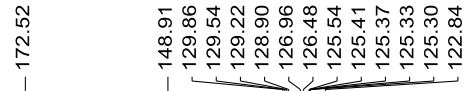

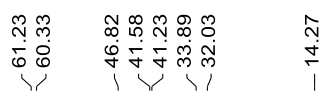<smiles>CCOCC[C@H]1CCN[C@H](c2ccc(C(F)(F)F)cc2)C1</smiles>

$2 a$

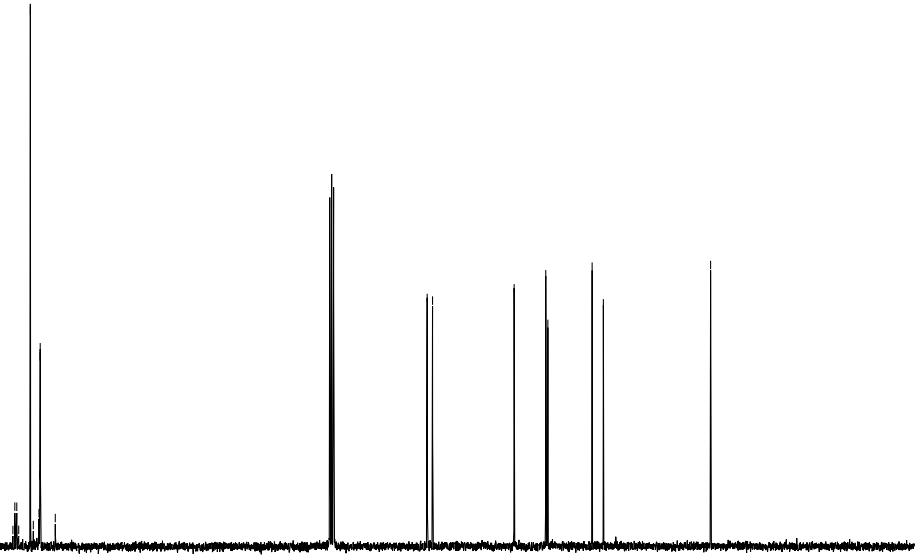

$\begin{array}{llllllllllllllllllllllll}1 & 1 & 200 & 190 & 180 & 170 & 160 & 150 & 140 & 130 & 120 & 110 & 100 & 90 & 80 & 70 & 60 & 50 & 40 & 30 & 20 & 10 & 0 & -10\end{array}$ f1 (ppm) 
${ }^{19} \mathrm{~F} \mathrm{NMR}$ of $2 \mathbf{a}\left(376 \mathrm{MHz}, \mathrm{CDCl}_{3}\right)$

兽
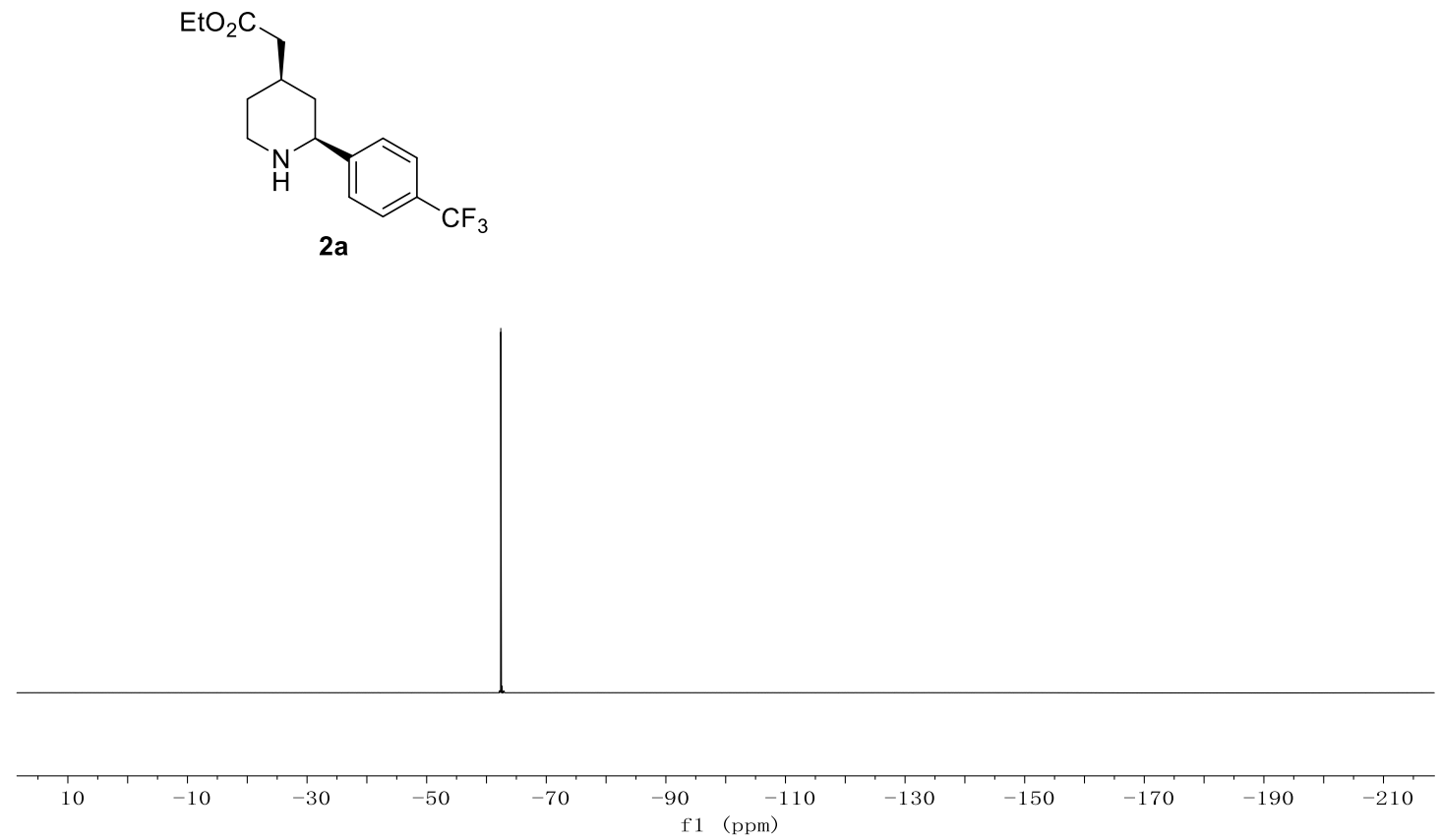
${ }^{1} \mathrm{H}$ NMR of $13 \mathbf{c}\left(400 \mathrm{MHz}, \mathrm{CDCl}_{3}\right)$

策

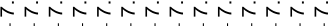

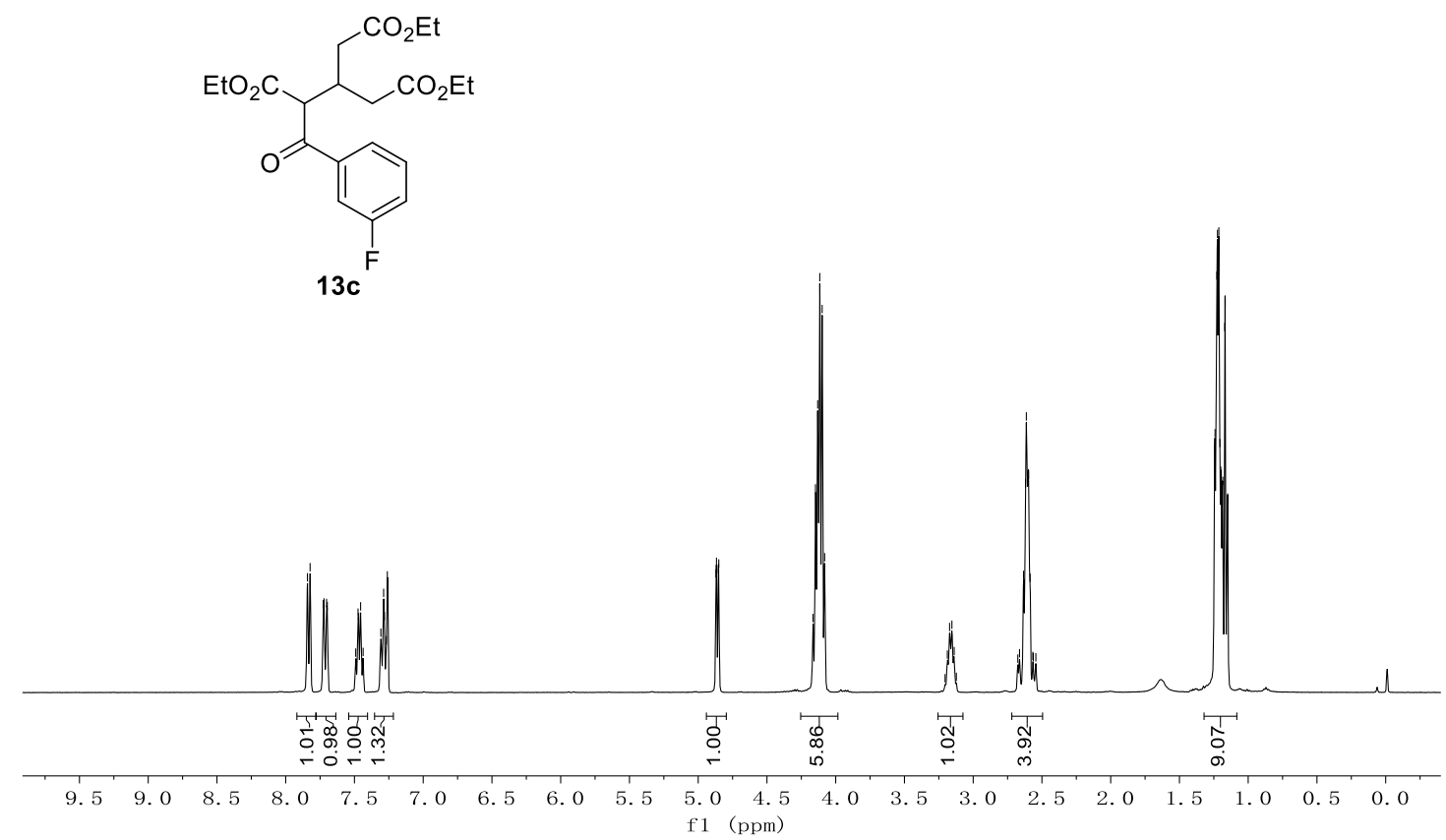

${ }^{13} \mathrm{C}\left\{{ }^{1} \mathrm{H}\right\}$ NMR of $13 \mathrm{c}\left(100 \mathrm{MHz}, \mathrm{CDCl}_{3}\right)$

\begin{tabular}{|c|c|c|c|}
\hline 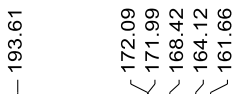 & 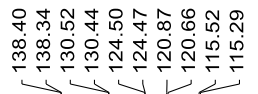 & 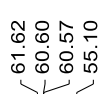 & 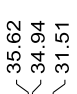 \\
\hline
\end{tabular}<smiles>CCOC(=O)CC(CC(=O)OCC)C(C(=O)OCC)C(=O)c1cccc(F)c1</smiles>

$\begin{array}{rllllllllllll}210 & 200 & 190 & 180 & 170 & 160 & 150 & 140 & 130 & 120 & 110 & 100 & 90 \\ \text { f1 } & (\mathrm{ppm})\end{array}$ 
${ }^{19} \mathrm{~F}$ NMR of $13 \mathrm{c}\left(376 \mathrm{MHz}, \mathrm{CDCl}_{3}\right)$

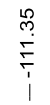
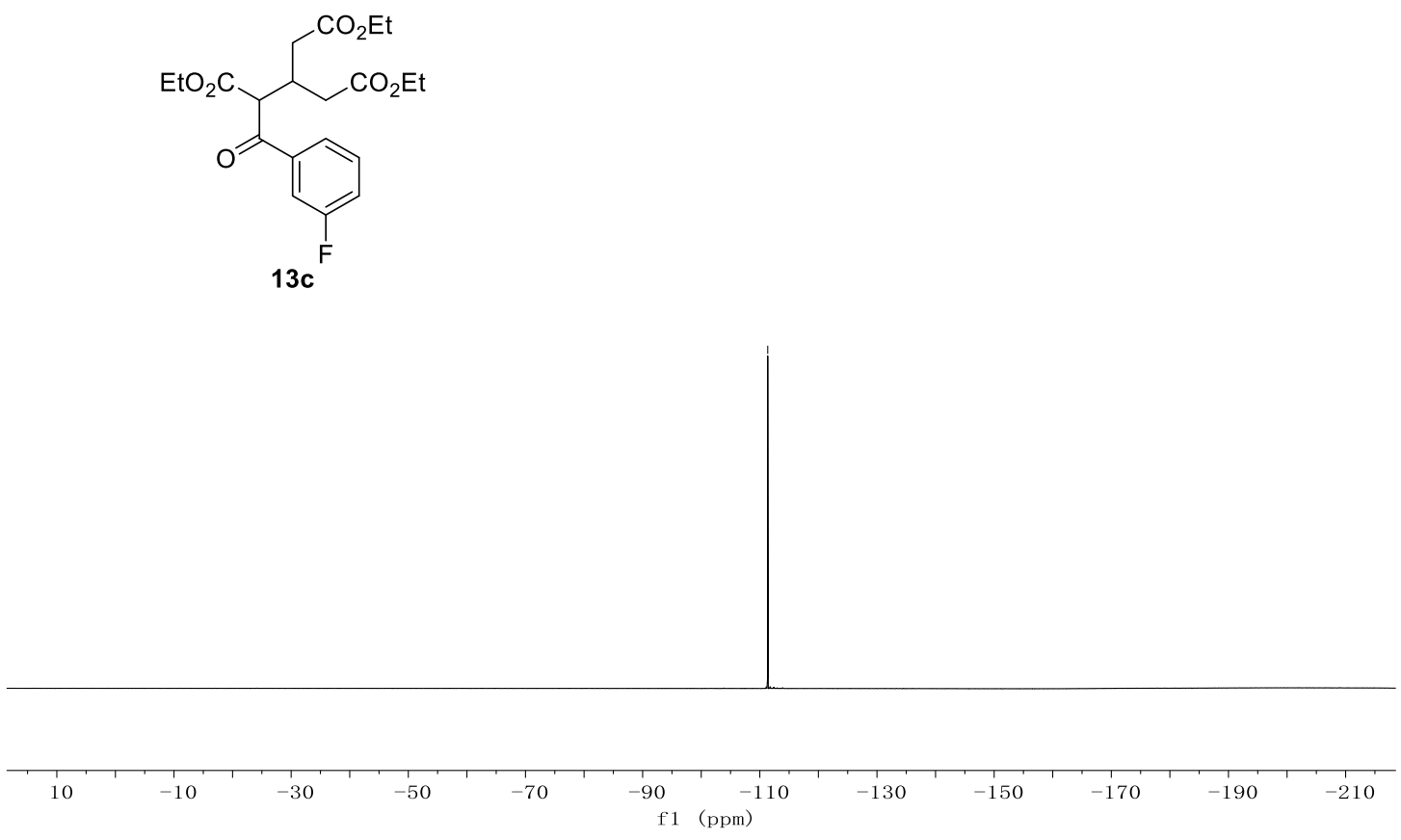
${ }^{1} \mathrm{H}$ NMR of $4 \mathbf{c}\left(400 \mathrm{MHz}, \mathrm{CDCl}_{3}\right)$

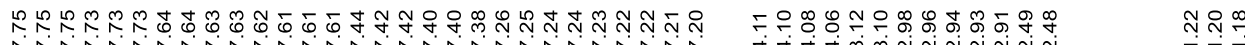

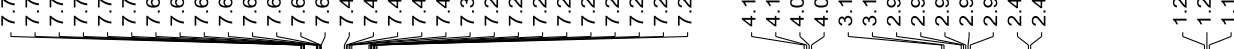

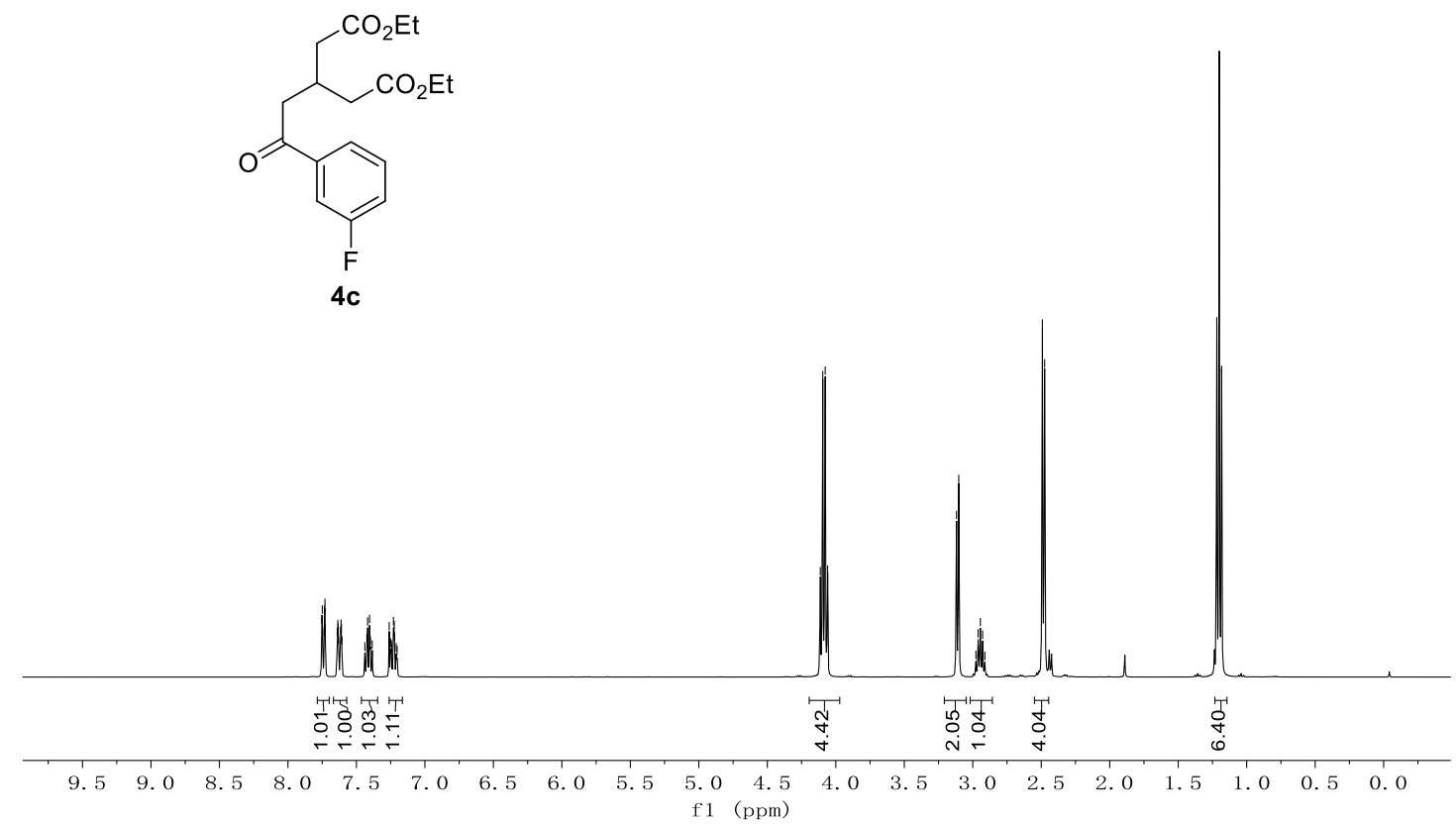

${ }^{13} \mathrm{C}\left\{{ }^{1} \mathrm{H}\right\} \mathrm{NMR}$ of $4 \mathrm{c}\left(100 \mathrm{MHz}, \mathrm{CDCl}_{3}\right)$

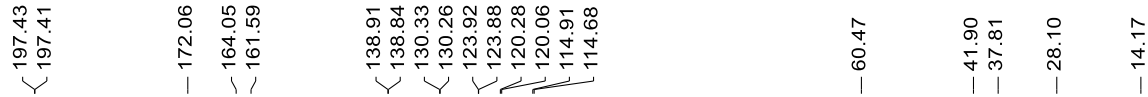<smiles>CCOC(=O)CC(CC(=O)OCC)CC(=O)c1cccc(C(F)(F)F)c1</smiles>

$\begin{array}{llllllllllllllllllllllll}210 & 200 & 190 & 180 & 170 & 160 & 150 & 140 & 130 & 120 & 110 & 100 & 90 & 80 & 70 & 60 & 50 & 40 & 30 & 20 & 10 & 0 & -10\end{array}$ f1 (ppm) 
${ }^{19} \mathrm{~F} \mathrm{NMR}$ of $4 \mathbf{c}\left(376 \mathrm{MHz}, \mathrm{CDCl}_{3}\right)$

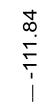

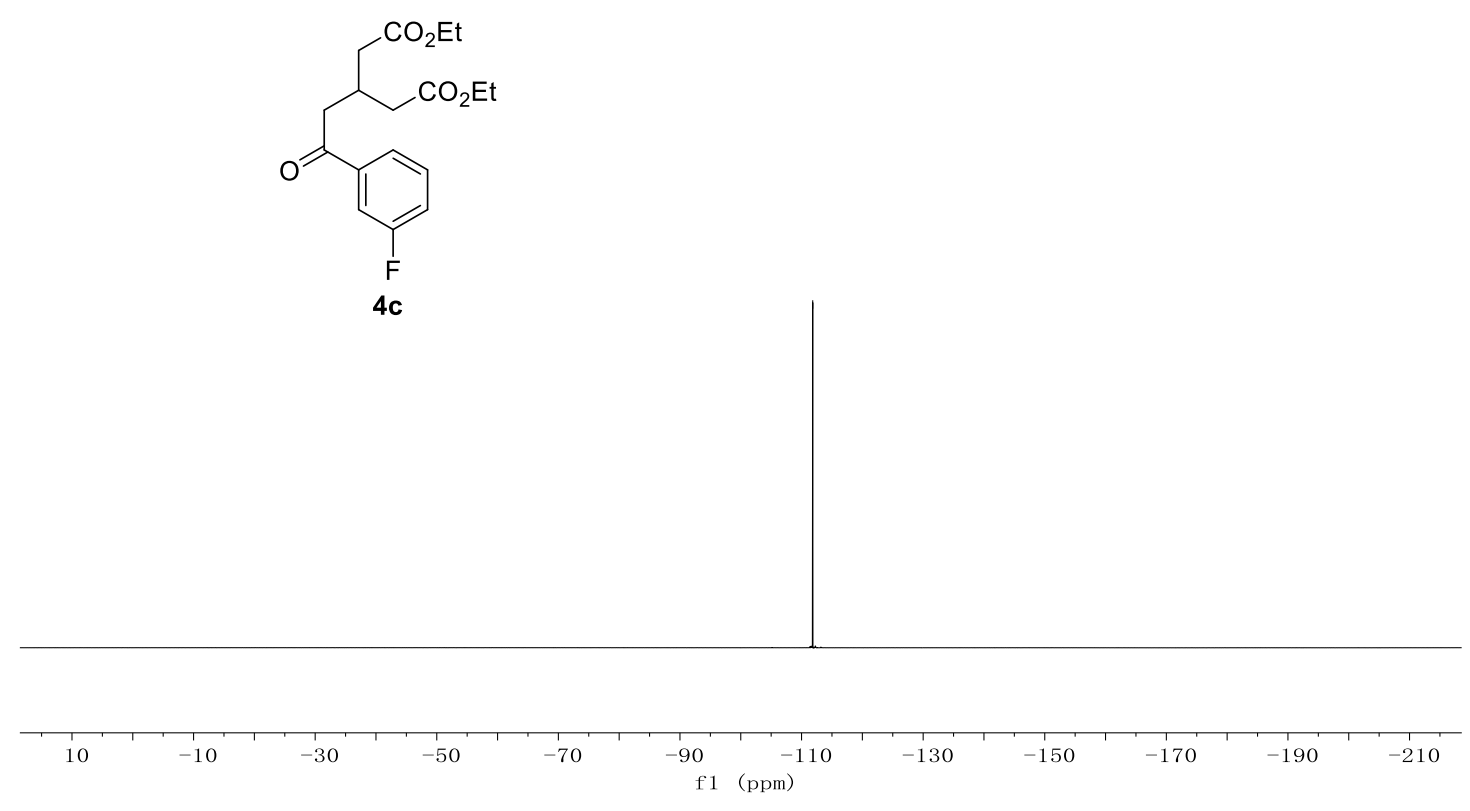


${ }^{1} \mathrm{H}$ NMR of $7 \mathbf{c}\left(400 \mathrm{MHz}, \mathrm{CDCl}_{3}\right)$

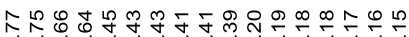

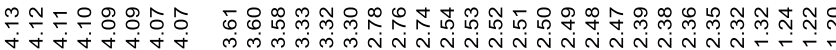

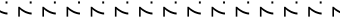<smiles>CCOCC(CC(=O)OCC)CC(=NS(=O)C(C)(C)C)c1cccc(F)c1</smiles>
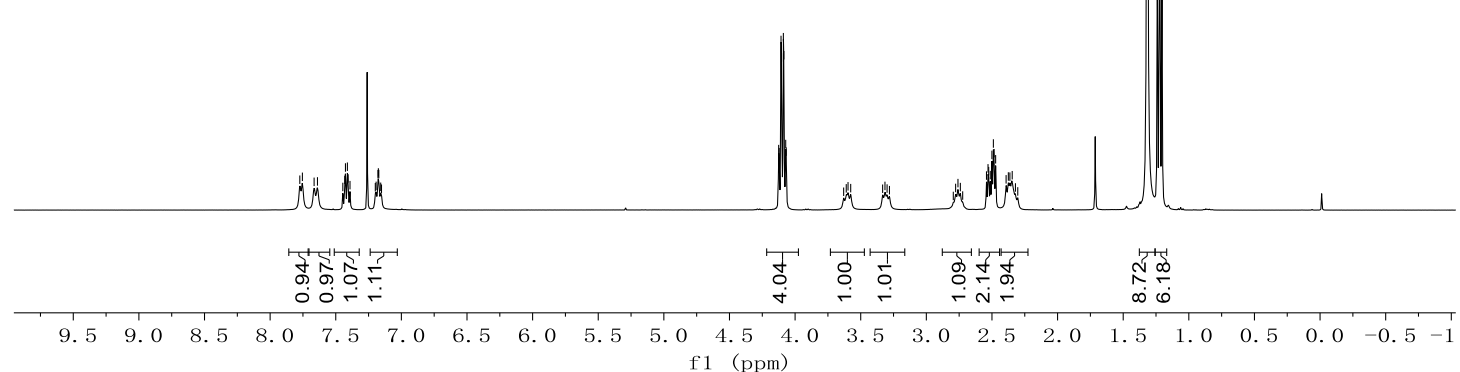

${ }^{13} \mathrm{C}\left\{{ }^{1} \mathrm{H}\right\}$ NMR of $7 \mathrm{c}\left(100 \mathrm{MHz}, \mathrm{CDCl}_{3}\right)$

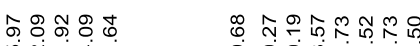

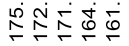

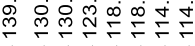

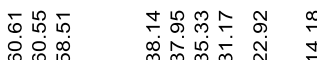

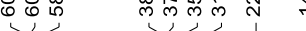<smiles>CCOC(=O)CC(CC(=O)OCC)CC(=NS(=O)C(C)(C)C)c1cccc(F)c1</smiles>

$7 c$

$\begin{array}{lllllllllllll}210 & 200 & 190 & 180 & 170 & 160 & 150 & 140 & 130 & 120 & 110 & 100 & 90\end{array}$ 
${ }^{19} \mathrm{~F} \mathrm{NMR}$ of $7 \mathbf{c}\left(376 \mathrm{MHz}, \mathrm{CDCl}_{3}\right)$

$$
\stackrel{\infty}{\stackrel{\infty}{\leftarrow}}
$$
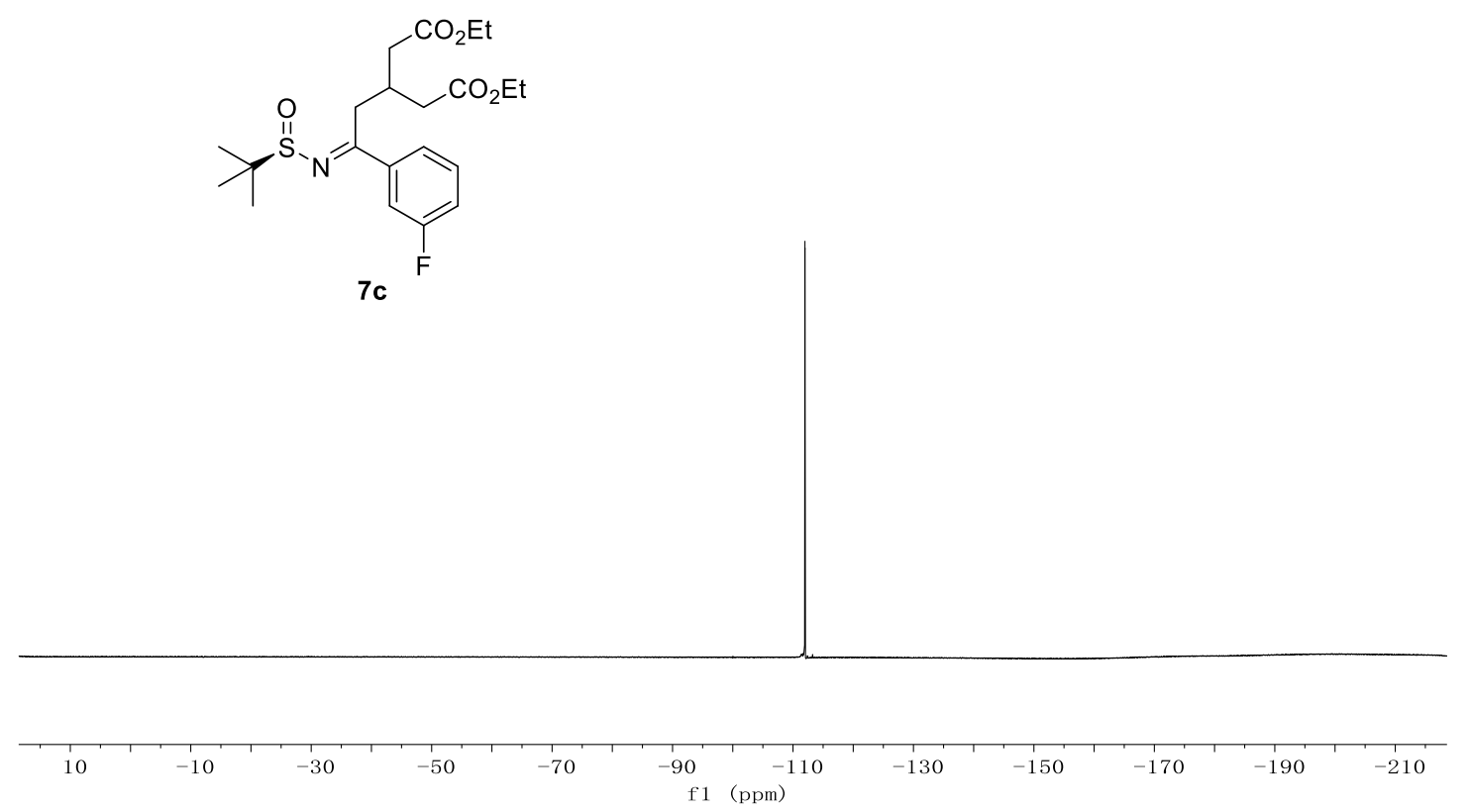
${ }^{1} \mathrm{H}$ NMR of $8 \mathbf{c}\left(400 \mathrm{MHz}, \mathrm{CDCl}_{3}\right)$

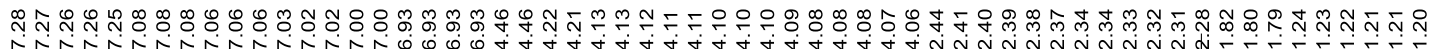

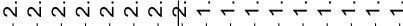<smiles>CCOC(=O)CC(CC(=O)OCC)CC(NS(=O)C(C)(C)C)c1cccc(F)c1</smiles>

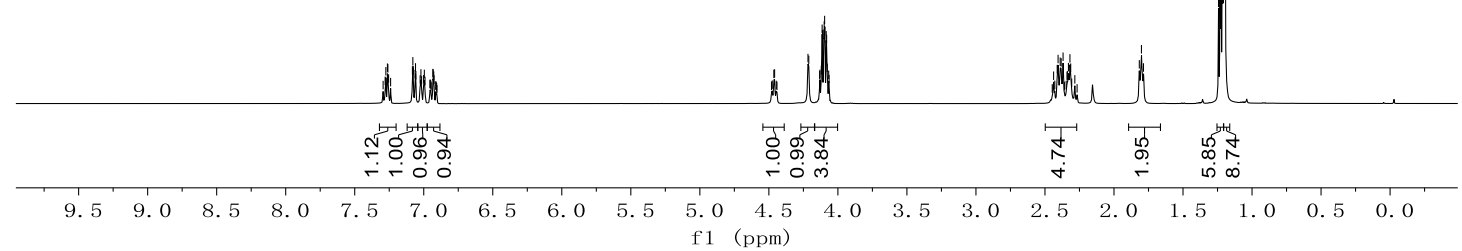

${ }^{13} \mathrm{C}\left\{{ }^{1} \mathrm{H}\right\}$ NMR of $8 \mathrm{c}\left(100 \mathrm{MHz}, \mathrm{CDCl}_{3}\right)$

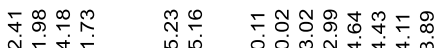

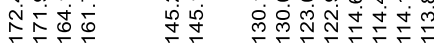

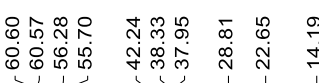<smiles>CCOC(=O)CC(CC(=O)OCC)CC(NS(=O)C(C)(C)C)c1cccc(F)c1</smiles>

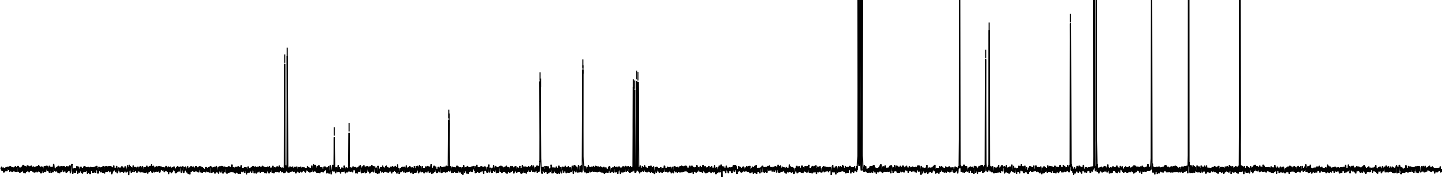

$\begin{array}{llllllllllllllllllllllll}210 & 200 & 190 & 180 & 170 & 160 & 150 & 140 & 130 & 120 & 110 & 100 & 90 & 80 & 70 & 60 & 50 & 40 & 30 & 20 & 10 & 0 & -10\end{array}$ f1 (ppm) 
${ }^{19} \mathrm{~F} \mathrm{NMR}$ of $8 \mathrm{c}\left(376 \mathrm{MHz}, \mathrm{CDCl}_{3}\right)$

$\stackrel{\infty}{0}$
$\stackrel{0}{N}$

i<smiles>CCOC(=O)CC(CC(=O)OCC)Cc1cccc(F)c1</smiles>

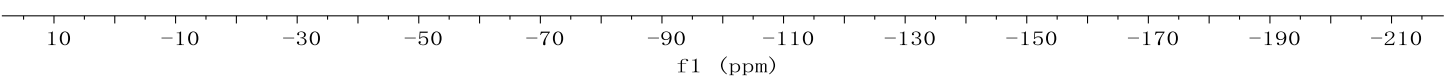


${ }^{1} \mathrm{H}$ NMR of $\mathbf{6 c}\left(400 \mathrm{MHz}, \mathrm{CDCl}_{3}\right)$

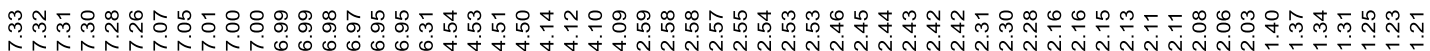
nis n n n<smiles>CCOC(=O)CC1CC(=O)N[C@H](c2cccc(F)c2)C1</smiles>

${ }^{13} \mathrm{C}\left\{{ }^{1} \mathrm{H}\right\}$ NMR of $6 \mathbf{c}\left(100 \mathrm{MHz}, \mathrm{CDCl}_{3}\right)$

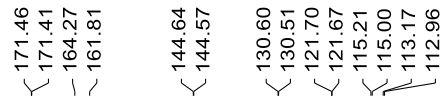

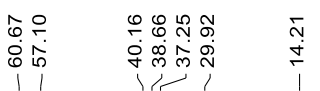<smiles>CCOC(=O)CC1CC(=O)N[C@H](c2cccc(F)c2)C1</smiles>

$\begin{array}{lllllllllllll}210 & 200 & 190 & 180 & 170 & 160 & 150 & 140 & 130 & 120 & 110 & 100 & 90 \\ \mathrm{f} 1 & (\mathrm{ppm})\end{array}$ 
${ }^{19} \mathrm{~F} \mathrm{NMR}$ of $\mathbf{6 c}\left(376 \mathrm{MHz}, \mathrm{CDCl}_{3}\right)$

$$
\text { 品 }
$$
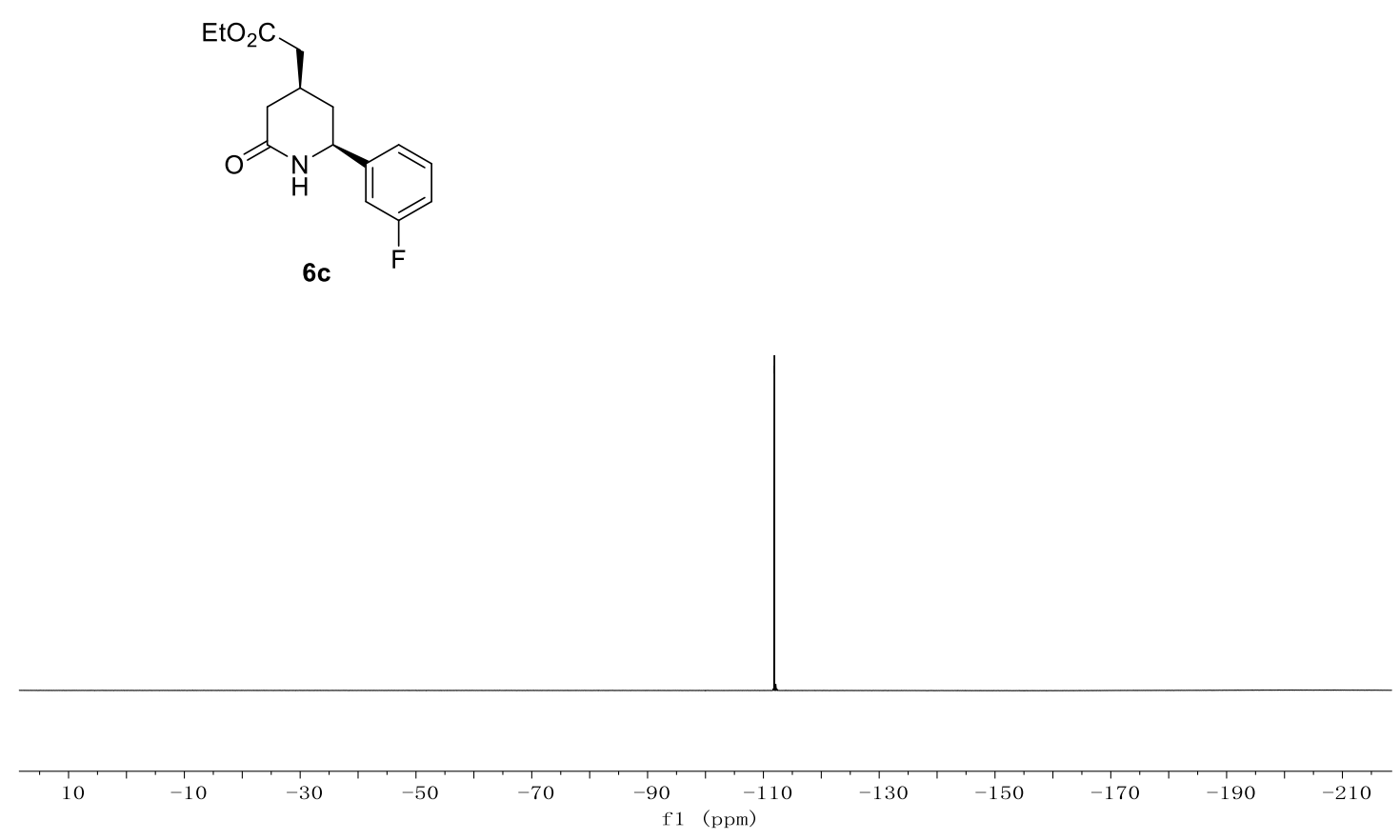
${ }^{1} \mathrm{H}$ NMR of $13 f\left(400 \mathrm{MHz}, \mathrm{CDCl}_{3}\right)$

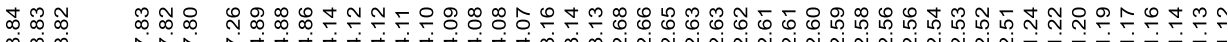

$\underbrace{\infty}_{0} \sqrt{i j}$

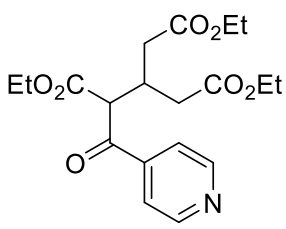

$13 f$

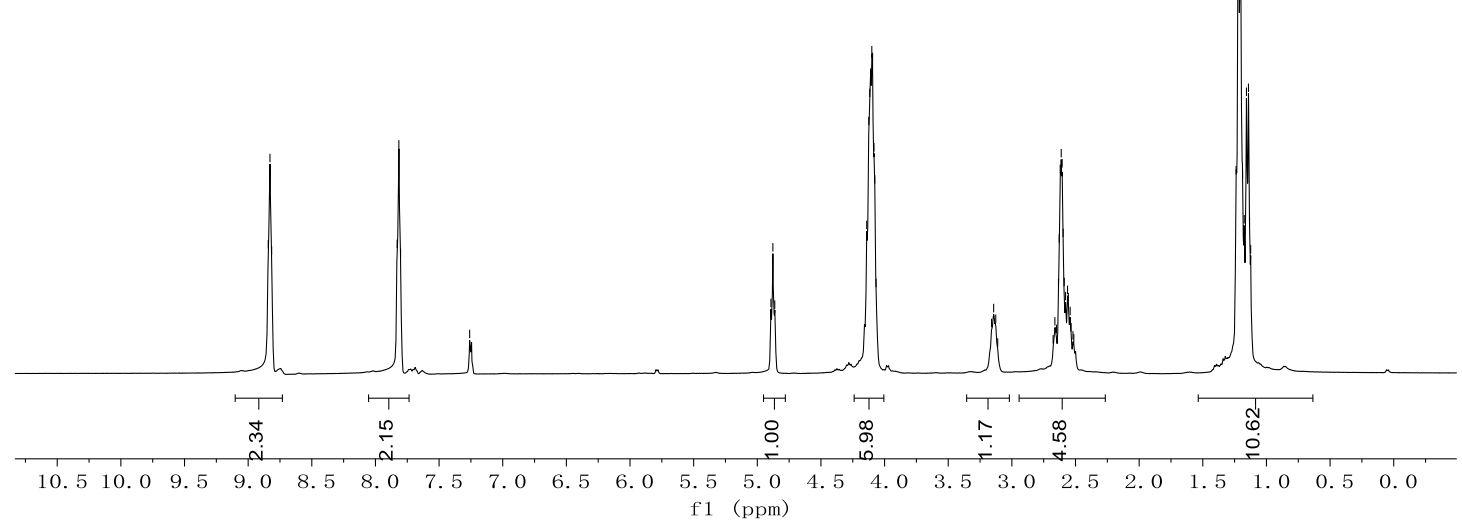

${ }^{13} \mathrm{C}\left\{{ }^{1} \mathrm{H}\right\}$ NMR of $13 f\left(100 \mathrm{MHz}, \mathrm{CDCl}_{3}\right)$

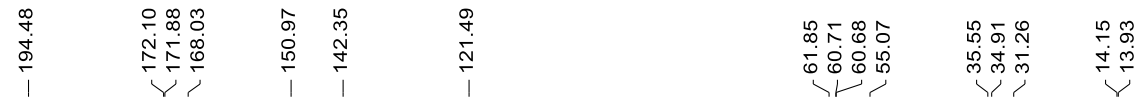<smiles>CCOC(=O)CC(CC(=O)OCC)C(C(=O)OCC)C(=O)c1ccncc1</smiles>

$13 f$

$\begin{array}{lllllllllllll}210 & 200 & 190 & 180 & 170 & 160 & 150 & 140 & 130 & 120 & 110 & 100 & 90\end{array}$ 
${ }^{1} \mathrm{H}$ NMR of $\mathbf{4 f}\left(400 \mathrm{MHz}, \mathrm{CDCl}_{3}\right)$

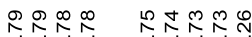

ionion ininis

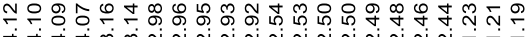

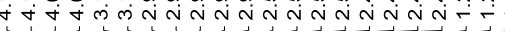
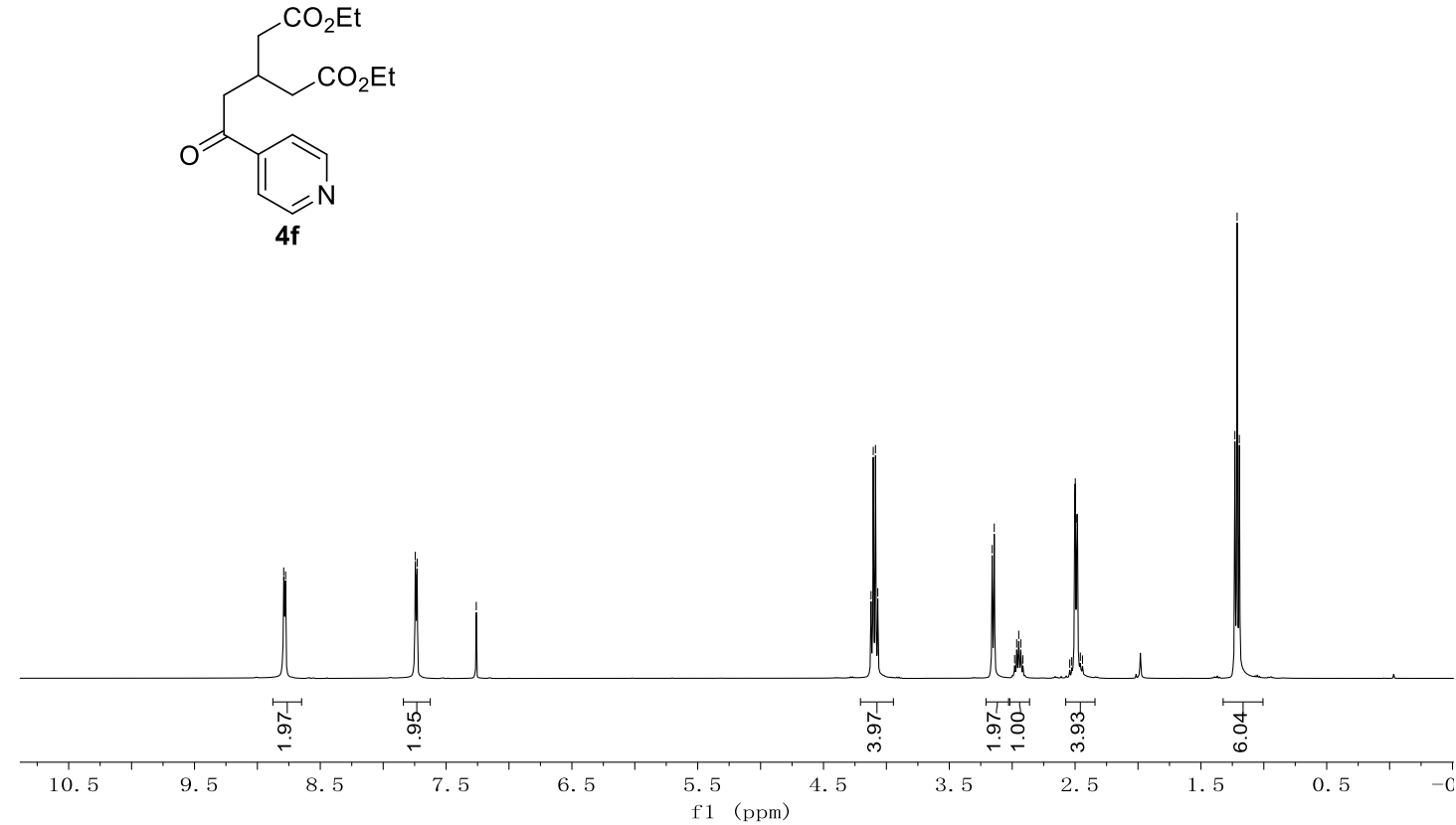

${ }^{13} \mathrm{C}\left\{{ }^{1} \mathrm{H}\right\}$ NMR of $\mathbf{4 f}\left(100 \mathrm{MHz}, \mathrm{CDCl}_{3}\right)$

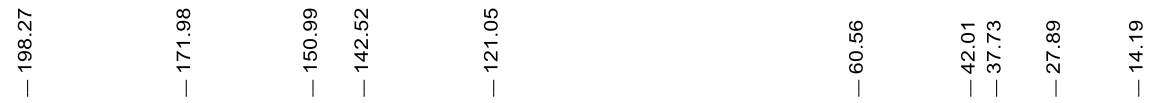<smiles>CCOC(=O)CC(CC(=O)OCC)CC(=O)c1ccncc1</smiles>

$\begin{array}{lllllllllllll}210 & 200 & 190 & 180 & 170 & 160 & 150 & 140 & 130 & 120 & 110 & 100 & 90\end{array}$ f1 (ppm) 
${ }^{1} \mathrm{H}$ NMR of $7 \mathbf{f}\left(400 \mathrm{MHz}, \mathrm{CDCl}_{3}\right)$<smiles>CCOC(=O)CC(CC(=O)OCC)CC(=NS(=O)C(C)(C)C)c1ccncc1</smiles>

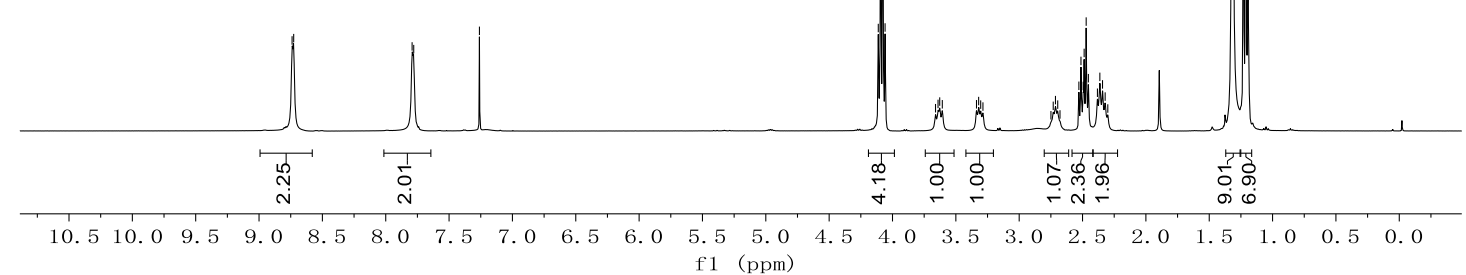

${ }^{13} \mathrm{C}\left\{{ }^{1} \mathrm{H}\right\} \mathrm{NMR}$ of $7 \mathbf{f}\left(100 \mathrm{MHz}, \mathrm{CDCl}_{3}\right)$

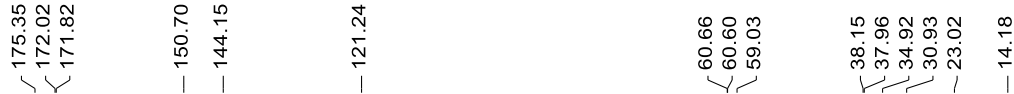<smiles>CCOC(=O)CC(CC(=O)OCC)CC(=NS(=O)C(C)(C)C)c1ccncc1</smiles> 
${ }^{1} \mathrm{H}$ NMR of $\mathbf{8 f}\left(400 \mathrm{MHz}, \mathrm{CDCl}_{3}\right)$

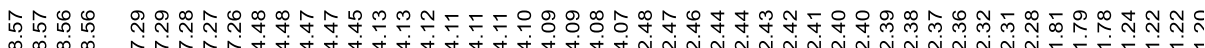

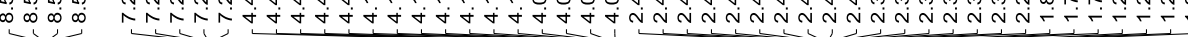<smiles>CCOC(=O)CC(CCOC)CC(NS(=O)C(C)(C)C)c1ccncc1</smiles>

$8 f$

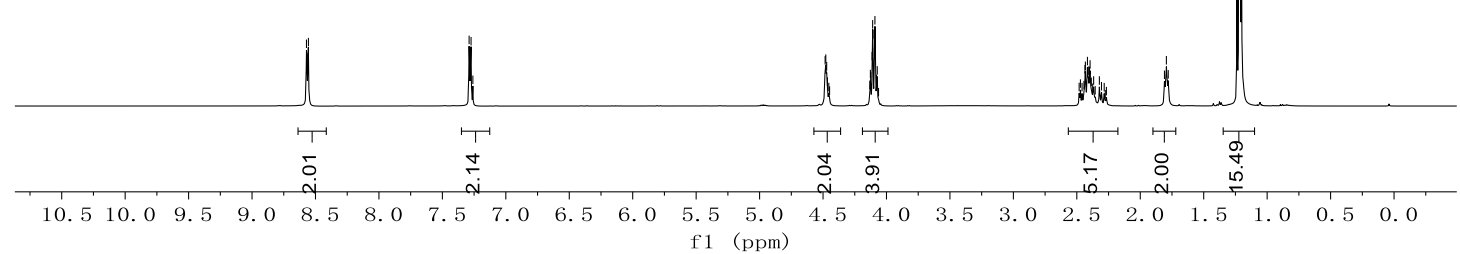

${ }^{13} \mathrm{C}\left\{{ }^{1} \mathrm{H}\right\} \mathrm{NMR}$ of $\mathbf{8 f}\left(100 \mathrm{MHz}, \mathrm{CDCl}_{3}\right)$

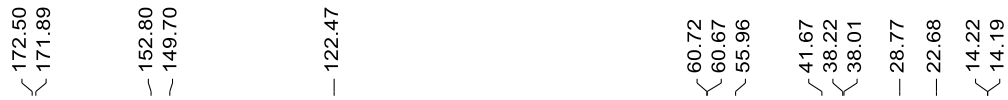<smiles>CCOCC(CC(=O)OCC)CC(NS(=O)C(C)(C)C)c1ccncc1</smiles>

$8 f$

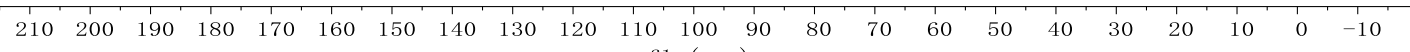
f1 (ppm) 
${ }^{1} \mathrm{H}$ NMR of $\mathbf{6 f}\left(400 \mathrm{MHz}, \mathrm{CDCl}_{3}\right)$

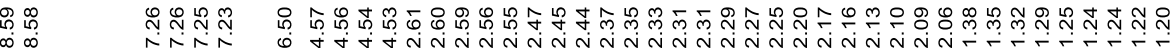

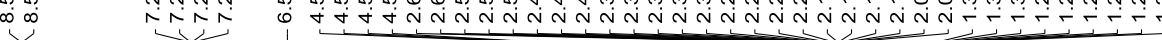

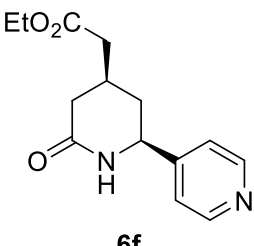

$6 f$

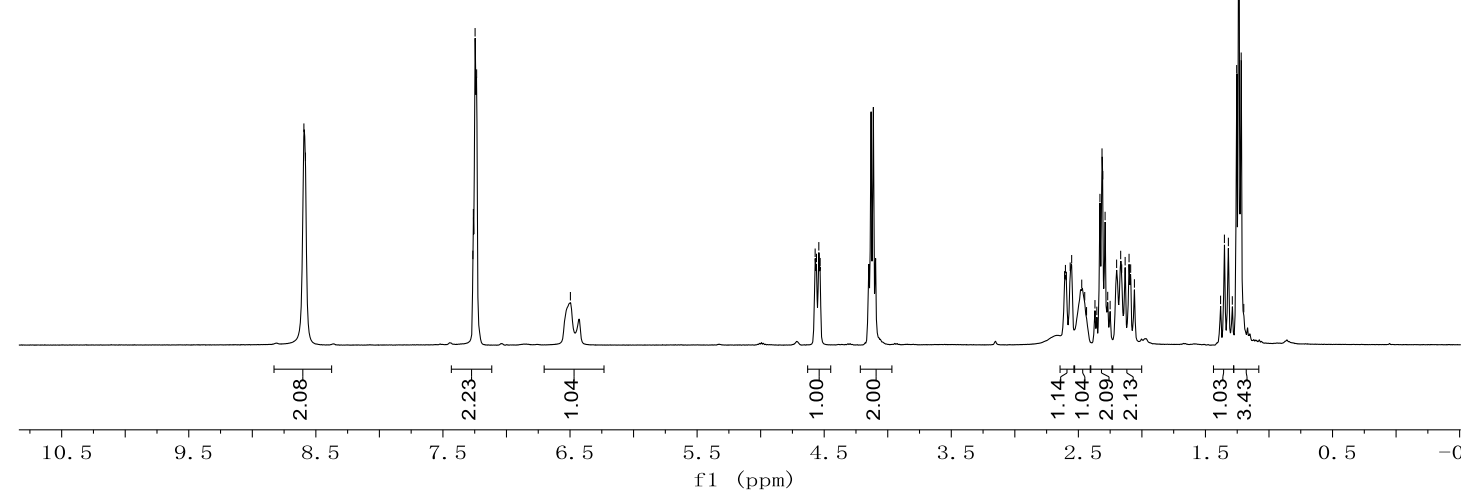

${ }^{13} \mathrm{C}\left\{{ }^{1} \mathrm{H}\right\}$ NMR of $6 \mathbf{f}\left(100 \mathrm{MHz}, \mathrm{CDCl}_{3}\right)$

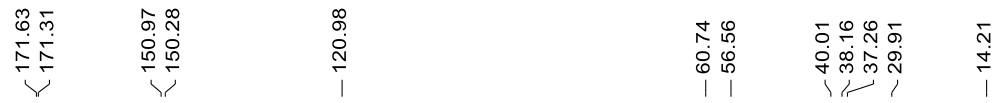<smiles>CCOC(=O)CC1CC(=O)NC(c2ccncc2)C1</smiles>

6f

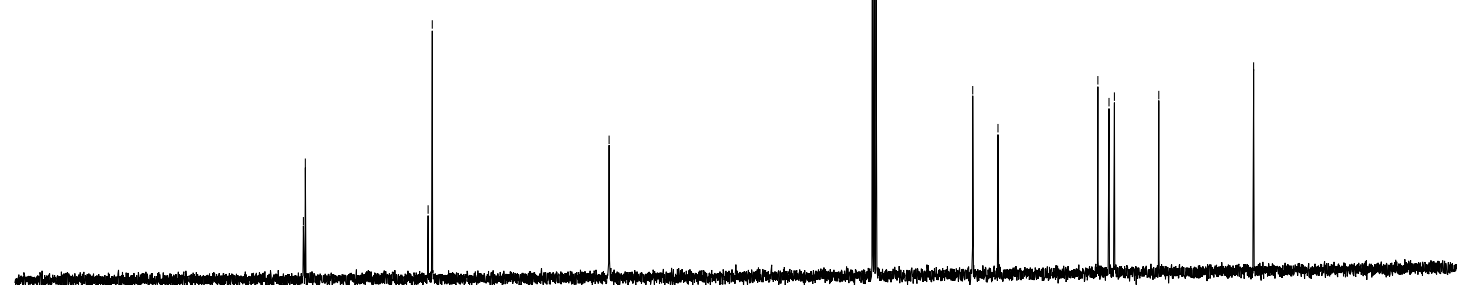

$\begin{array}{lllllllllllll}210 & 200 & 190 & 180 & 170 & 160 & 150 & 140 & 130 & 120 & 110 & 100 & 90\end{array}$ f1 (ppm) 
${ }^{1} \mathrm{H}$ NMR of $\mathbf{1 3 d}\left(400 \mathrm{MHz}, \mathrm{CDCl}_{3}\right)$

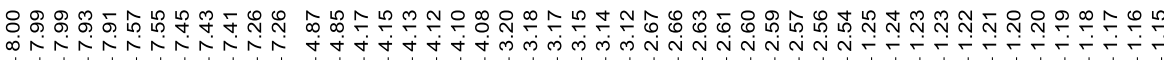

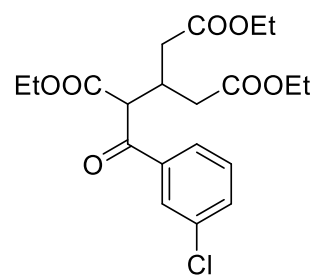

$13 d$

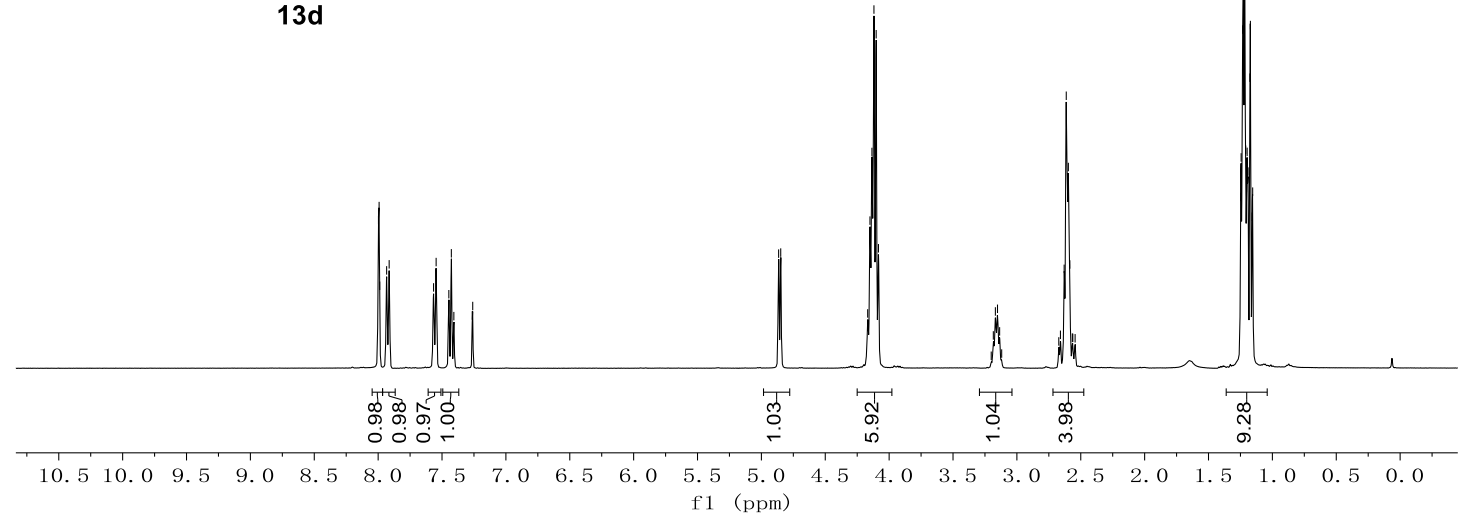

${ }^{13} \mathrm{C}\left\{{ }^{1} \mathrm{H}\right\}$ NMR of 13d $\left(100 \mathrm{MHz}, \mathrm{CDCl}_{3}\right)$

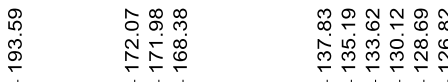

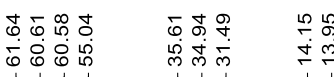<smiles>CCOC(=O)CC(CC(=O)OCC)C(C(=O)OCC)C(=O)c1cccc(Cl)c1</smiles>

13d

$\begin{array}{lllllllllllll}210 & 200 & 190 & 180 & 170 & 160 & 150 & 140 & 130 & 120 & 110 & 100 & 90\end{array}$ 
${ }^{1} \mathrm{H}$ NMR of $4 \mathbf{d}\left(400 \mathrm{MHz}, \mathrm{CDCl}_{3}\right)$

б б б

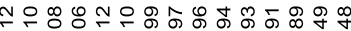

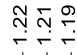

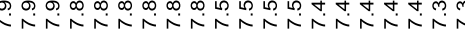
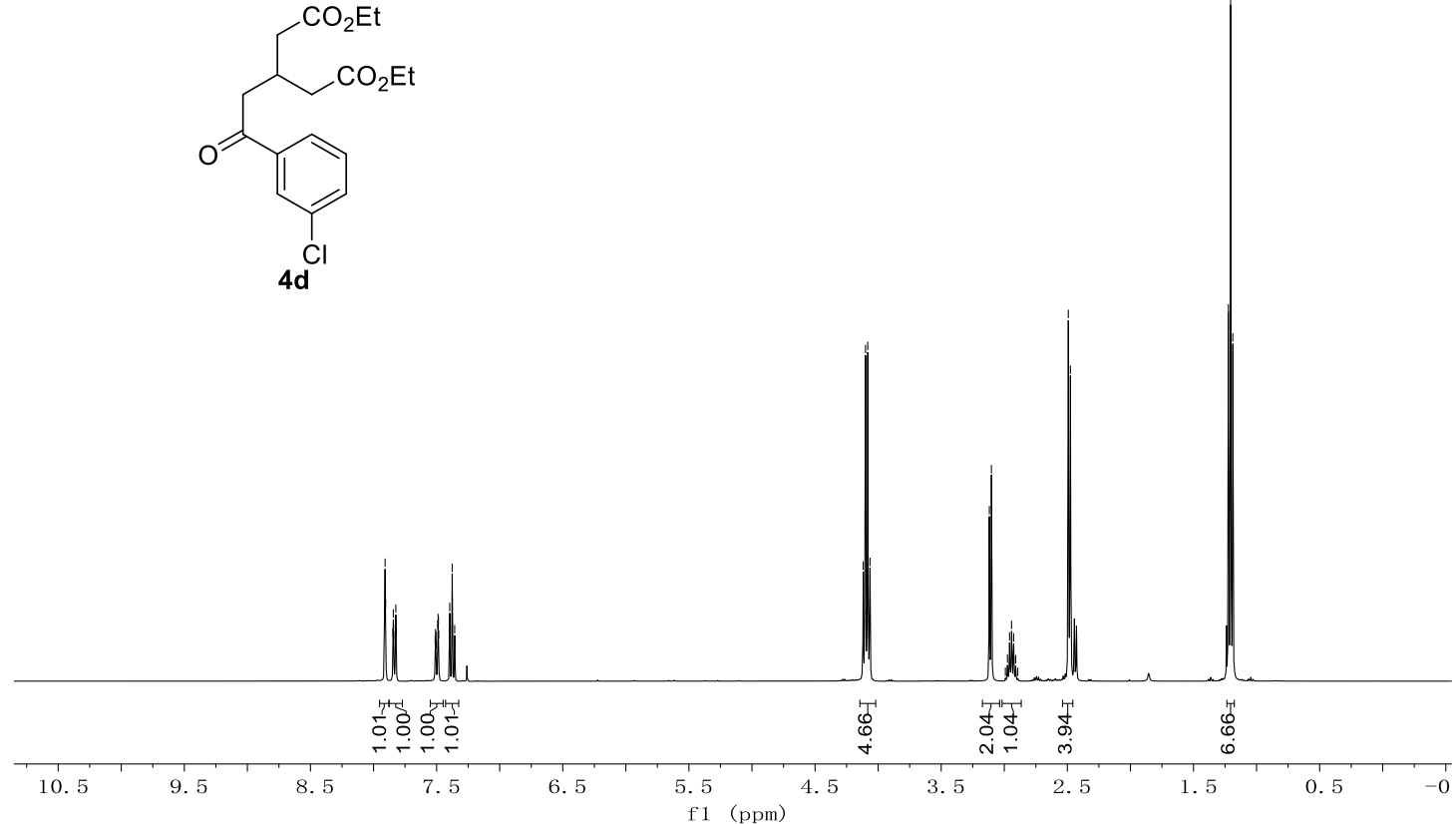

${ }^{13} \mathrm{C}\left\{{ }^{1} \mathrm{H}\right\}$ NMR of $\mathbf{4 d}\left(100 \mathrm{MHz}, \mathrm{CDCl}_{3}\right)$

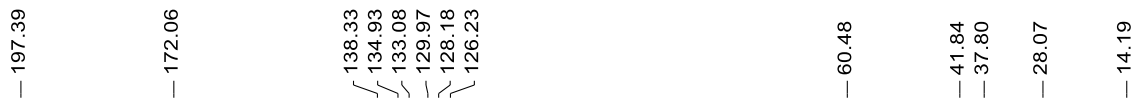

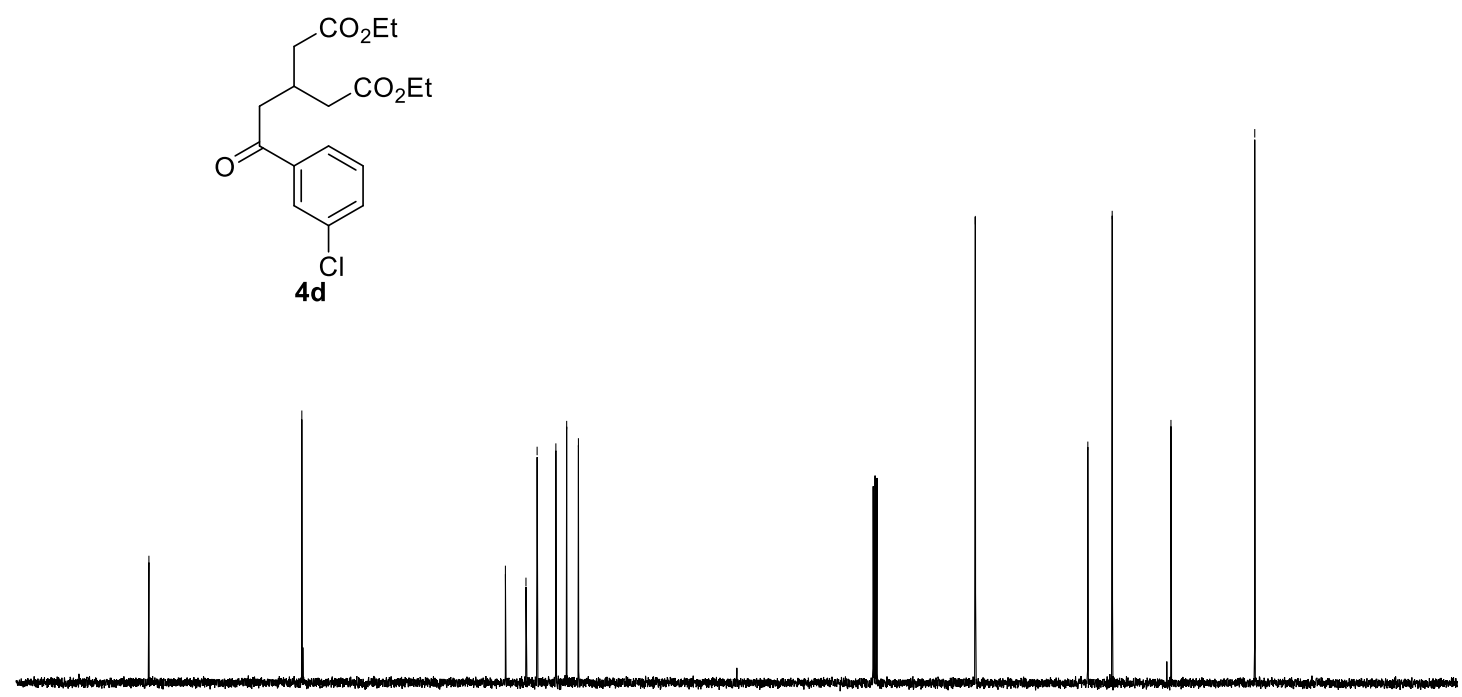

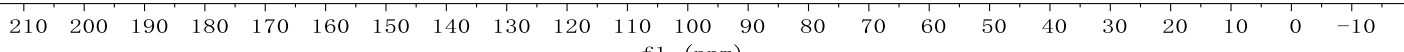
f1 (ppm) 
${ }^{1} \mathrm{H}$ NMR of $7 \mathbf{d}\left(400 \mathrm{MHz}, \mathrm{CDCl}_{3}\right)$

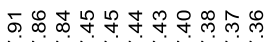

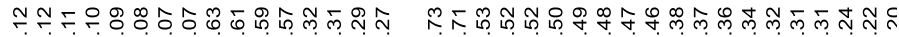

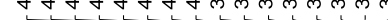

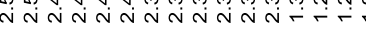<smiles>CCOC(=O)CC(CC(=O)OCC)CC(=N[S@](=O)C(C)(C)C)c1cccc(Cl)c1</smiles>

$7 d$

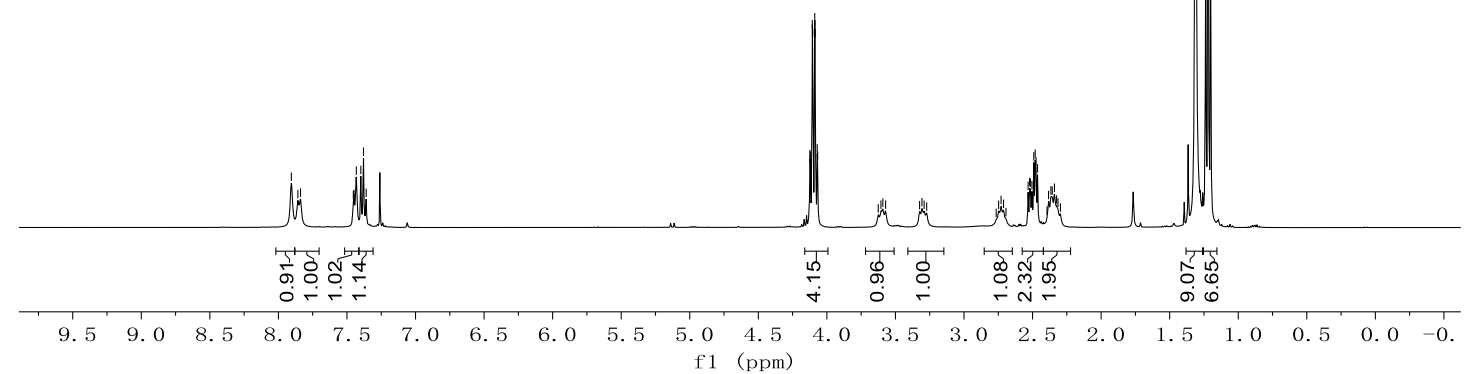

${ }^{13} \mathrm{C}\left\{{ }^{1} \mathrm{H}\right\}$ NMR of $7 \mathbf{d}\left(100 \mathrm{MHz}, \mathrm{CDCl}_{3}\right)$

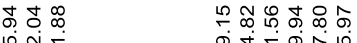

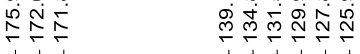

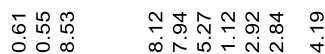

रोiा

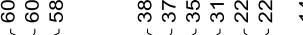<smiles>CCOCCC(CC(=O)OCC)CC(=N[S@](=O)C(C)(C)C)c1cccc(Cl)c1</smiles>

7d

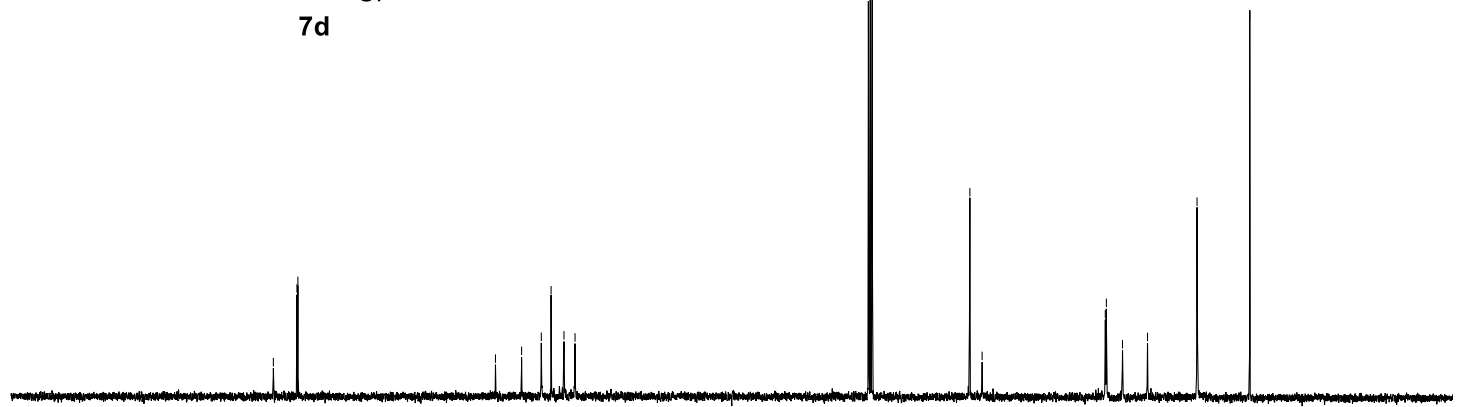

$\begin{array}{lllllllllllllllllllllll}210 & 200 & 190 & 180 & 170 & 160 & 150 & 140 & 130 & 120 & 110 & 100 & 90 & 80 & 70 & 60 & 50 & 40 & 30 & 20 & 10 & 0 & -10\end{array}$ f1 (ppm) 
${ }^{1} \mathrm{H}$ NMR of $8 \mathbf{d}\left(400 \mathrm{MHz}, \mathrm{CDCl}_{3}\right)$

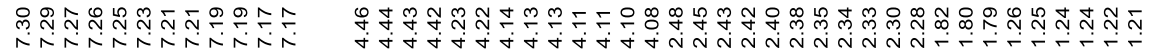<smiles>CCOC(=O)CC(CC(=O)OCC)Cc1cccc(Cl)c1</smiles>

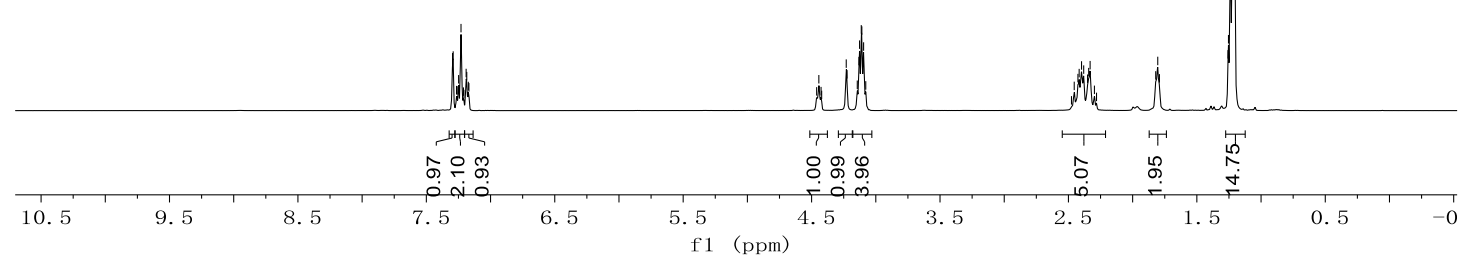

${ }^{13} \mathrm{C}\left\{{ }^{1} \mathrm{H}\right\}$ NMR of $8 \mathbf{d}\left(100 \mathrm{MHz}, \mathrm{CDCl}_{3}\right)$
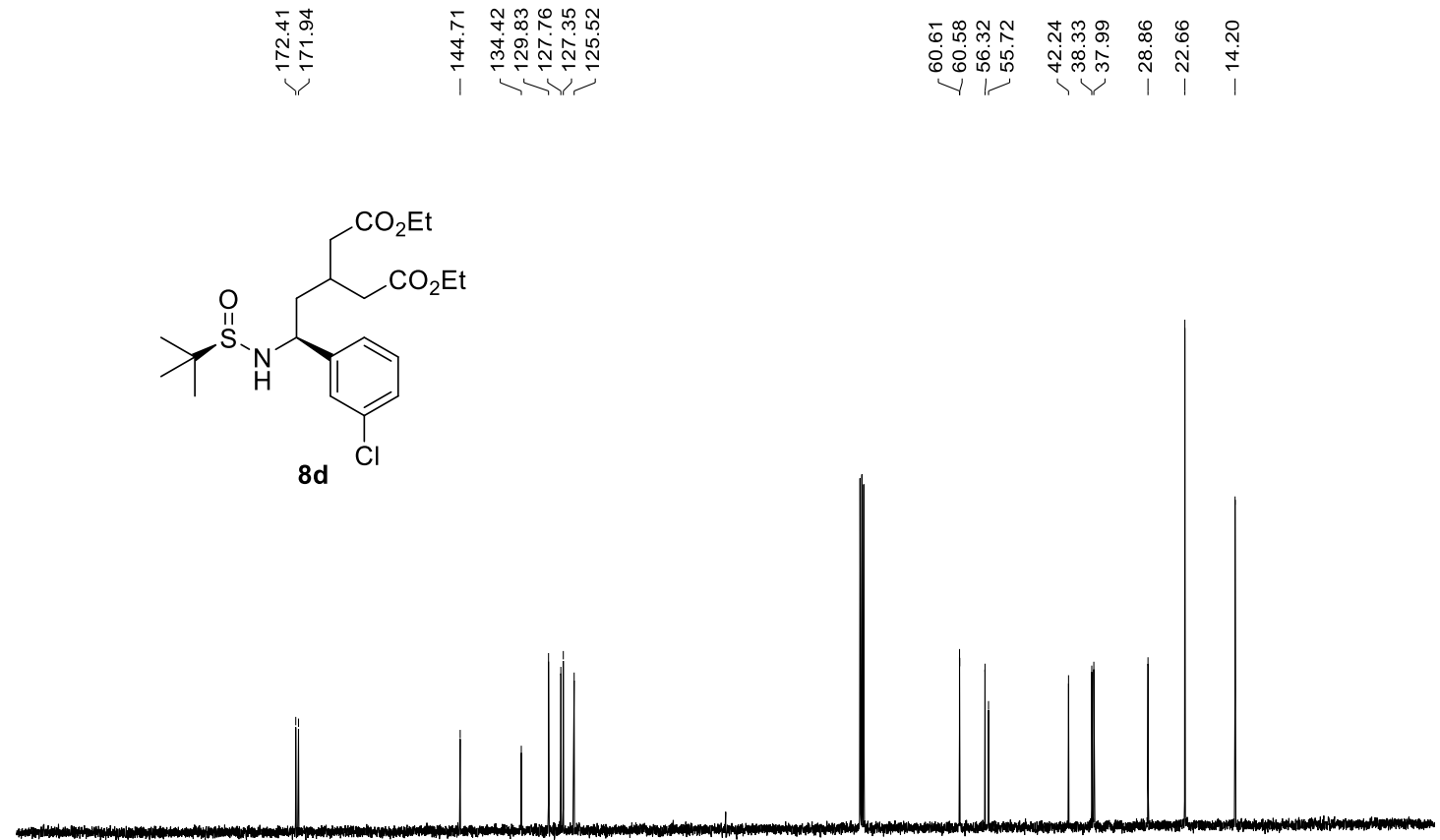

8d

$\begin{array}{lllllllllllll}210 & 200 & 190 & 180 & 170 & 160 & 150 & 140 & 130 & 120 & 110 & 100 & 90\end{array}$ 
${ }^{1} \mathrm{H}$ NMR of $\mathbf{6 d}\left(400 \mathrm{MHz}, \mathrm{CDCl}_{3}\right)$

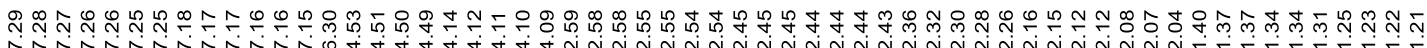

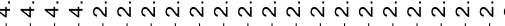

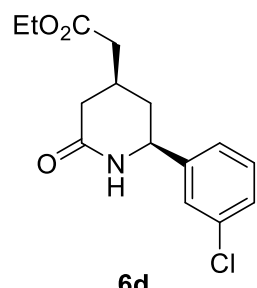

$6 d$

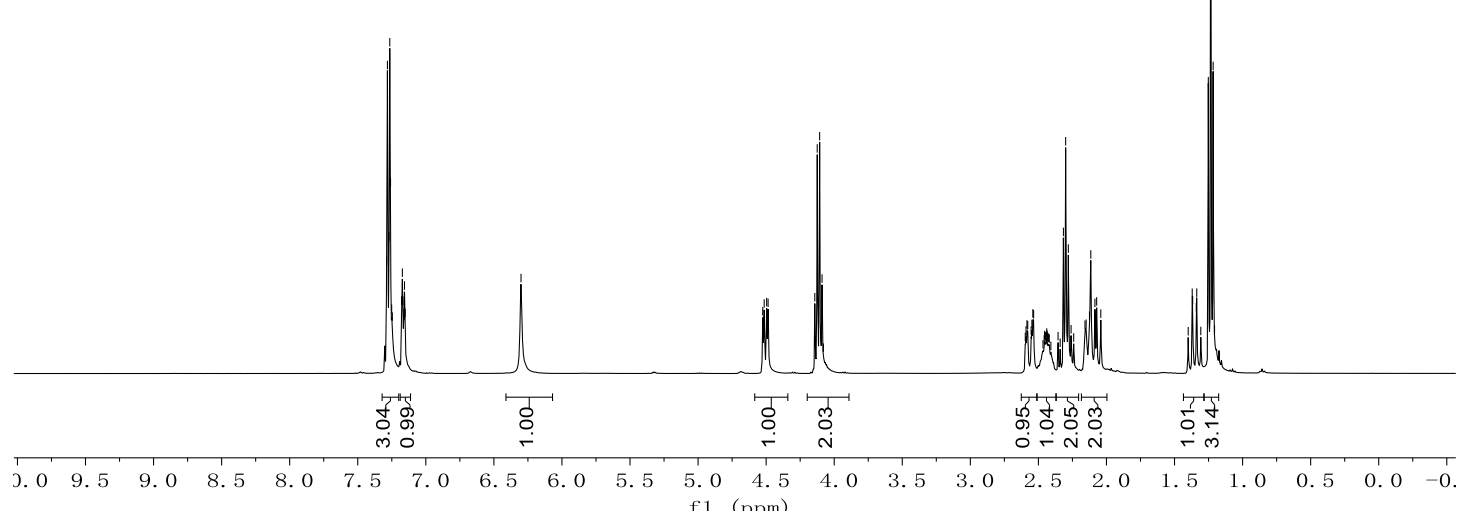

${ }^{13} \mathrm{C}\left\{{ }^{1} \mathrm{H}\right\}$ NMR of $\mathbf{6 d}\left(100 \mathrm{MHz}, \mathrm{CDCl}_{3}\right)$

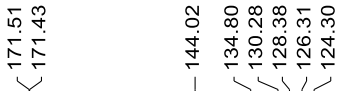

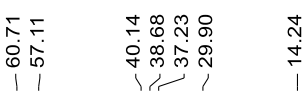<smiles>CCOCCC1CC(=O)N[C@H](c2cccc(Cl)c2)C1</smiles>

$6 d$

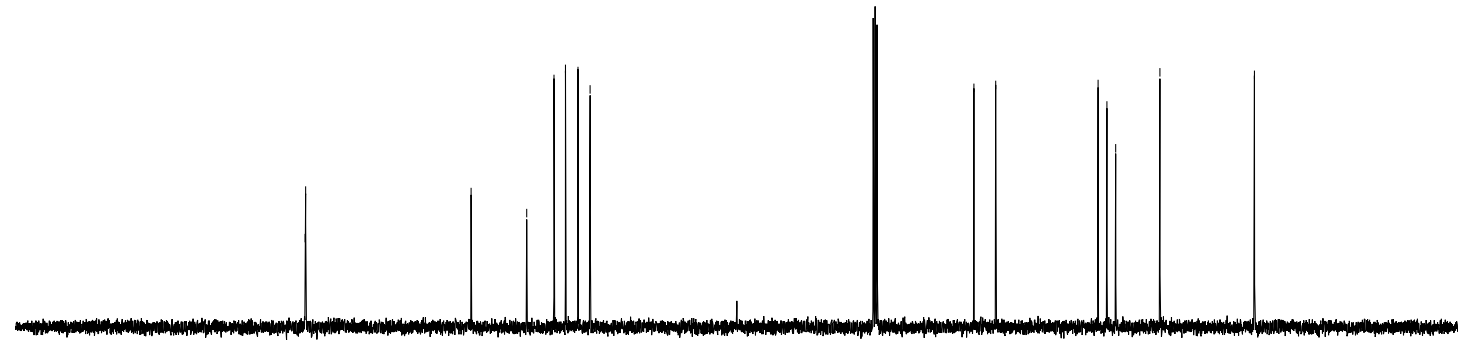

$\begin{array}{lllllllllllll}210 & 200 & 190 & 180 & 170 & 160 & 150 & 140 & 130 & 120 & 110 & 100 & 90\end{array}$ f1 (ppm) 
${ }^{1} \mathrm{H}$ NMR of $\mathbf{1 3 b}\left(400 \mathrm{MHz}, \mathrm{CDCl}_{3}\right)$

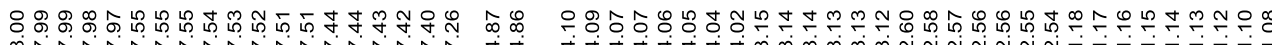

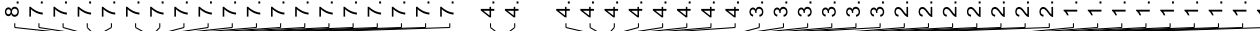

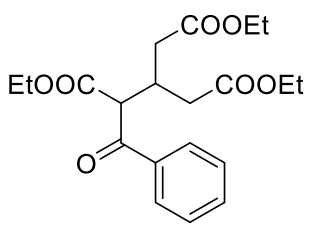

13b

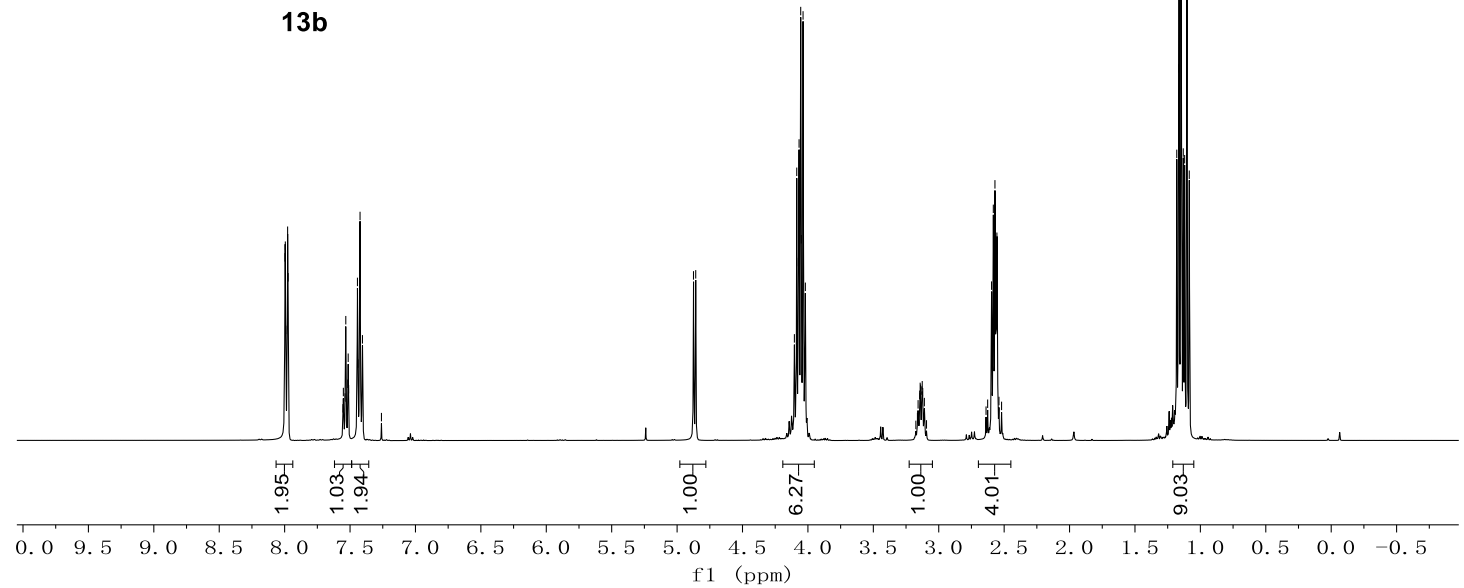

${ }^{13} \mathrm{C}\left\{{ }^{1} \mathrm{H}\right\}$ NMR of $\mathbf{1 3 b}\left(100 \mathrm{MHz}, \mathrm{CDCl}_{3}\right)$

\begin{tabular}{|c|c|c|c|}
\hline 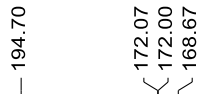 & 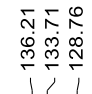 & 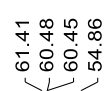 & 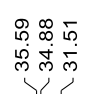 \\
\hline
\end{tabular}<smiles>CCOC(=O)CC(CC(=O)OCC)C(C(=O)OCC)C(=O)c1ccccc1</smiles>

$13 b$

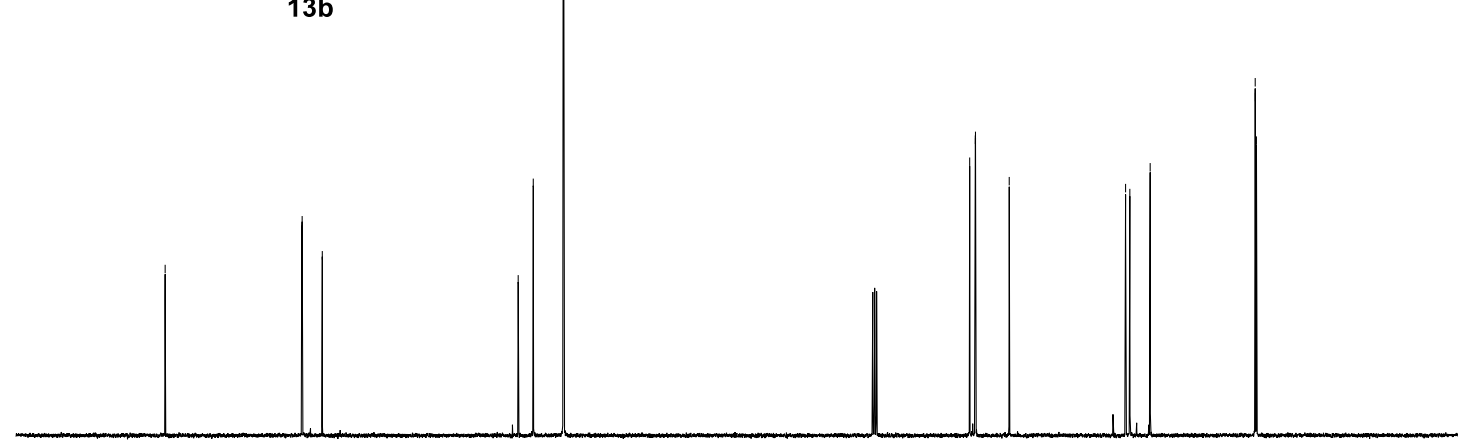

$\begin{array}{lllllllllllllllllllllllllllllll}210 & 200 & 190 & 180 & 170 & 160 & 150 & 140 & 130 & 120 & 110 & 100 & 90 & 80 & 70 & 60 & 50 & 40 & 30 & 20 & 10 & 0 & -10\end{array}$ 1 1 (ppm) 
${ }^{1} \mathrm{H}$ NMR of $\mathbf{4 b}\left(400 \mathrm{MHz}, \mathrm{CDCl}_{3}\right)$

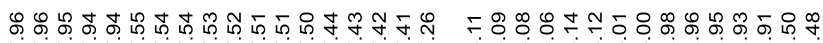

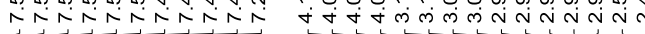

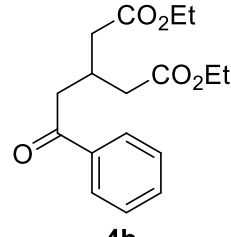

$4 \mathbf{b}$

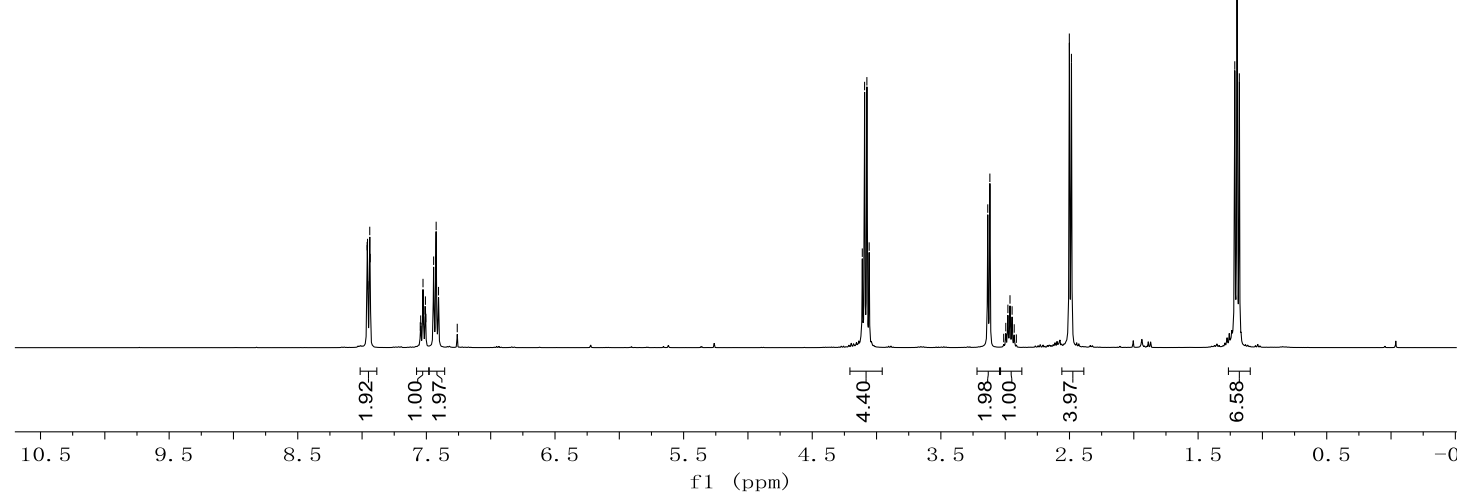

${ }^{13} \mathrm{C}\left\{{ }^{1} \mathrm{H}\right\}$ NMR of $\mathbf{4 b}\left(100 \mathrm{MHz}, \mathrm{CDCl}_{3}\right)$

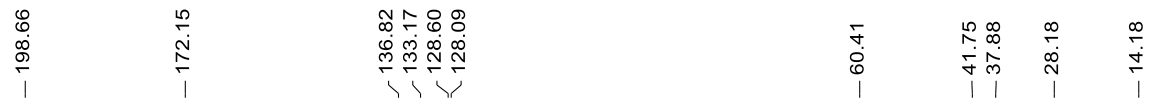<smiles>CCOC(=O)CC(CC(=O)OCC)CC(=O)c1ccccc1</smiles>

4b

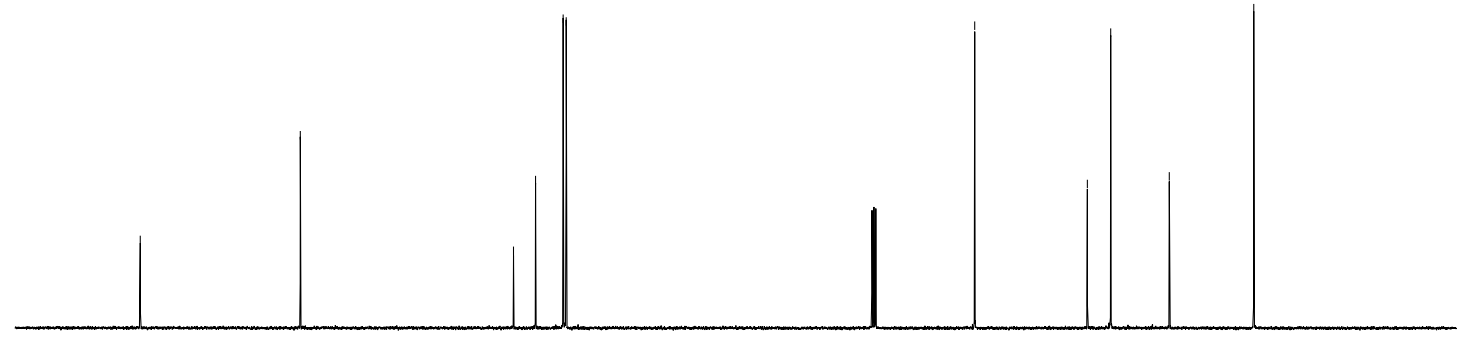

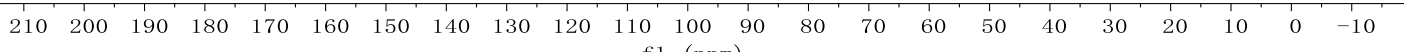
f1 (ppm) 
${ }^{1} \mathrm{H}$ NMR of $7 \mathbf{b}\left(400 \mathrm{MHz}, \mathrm{CDCl}_{3}\right)$

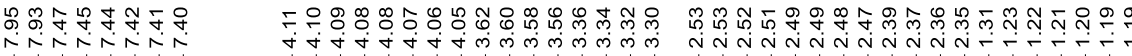

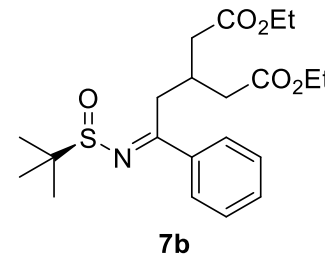

$7 b$

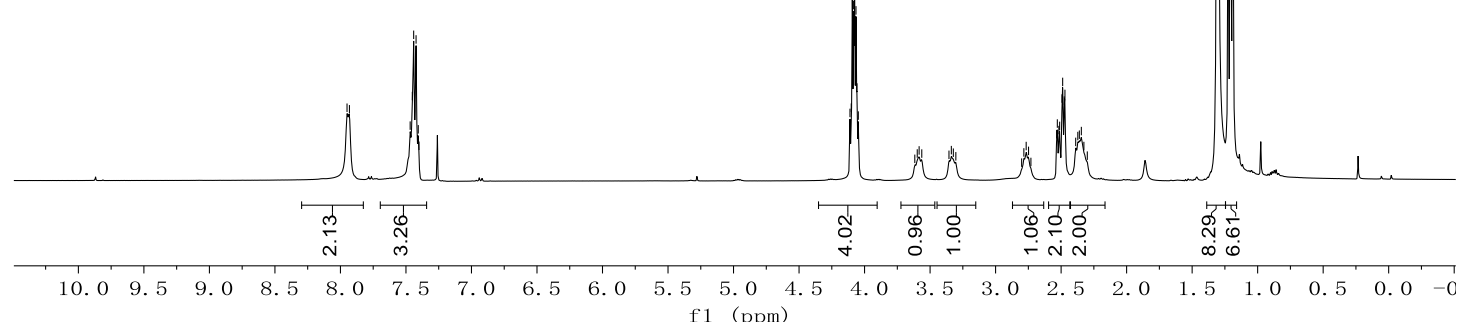

${ }^{13} \mathrm{C}\left\{{ }^{1} \mathrm{H}\right\}$ NMR of $7 \mathbf{b}\left(100 \mathrm{MHz}, \mathrm{CDCl}_{3}\right)$

ํㅗㄴ

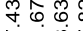



نำ

i资

$\mid>\backslash$

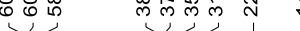<smiles>CCOC(=O)CC(CC(=O)OCC)CC(=NS(=O)C(C)(C)C)c1ccccc1</smiles>

$7 b$

$\begin{array}{lllllllllllll}210 & 200 & 190 & 180 & 170 & 160 & 150 & 140 & 130 & 120 & 110 & 100 & 90\end{array}$

f1 (ppm) 
${ }^{1} \mathrm{H}$ NMR of $\mathbf{8 b}\left(400 \mathrm{MHz}, \mathrm{CDCl}_{3}\right)$

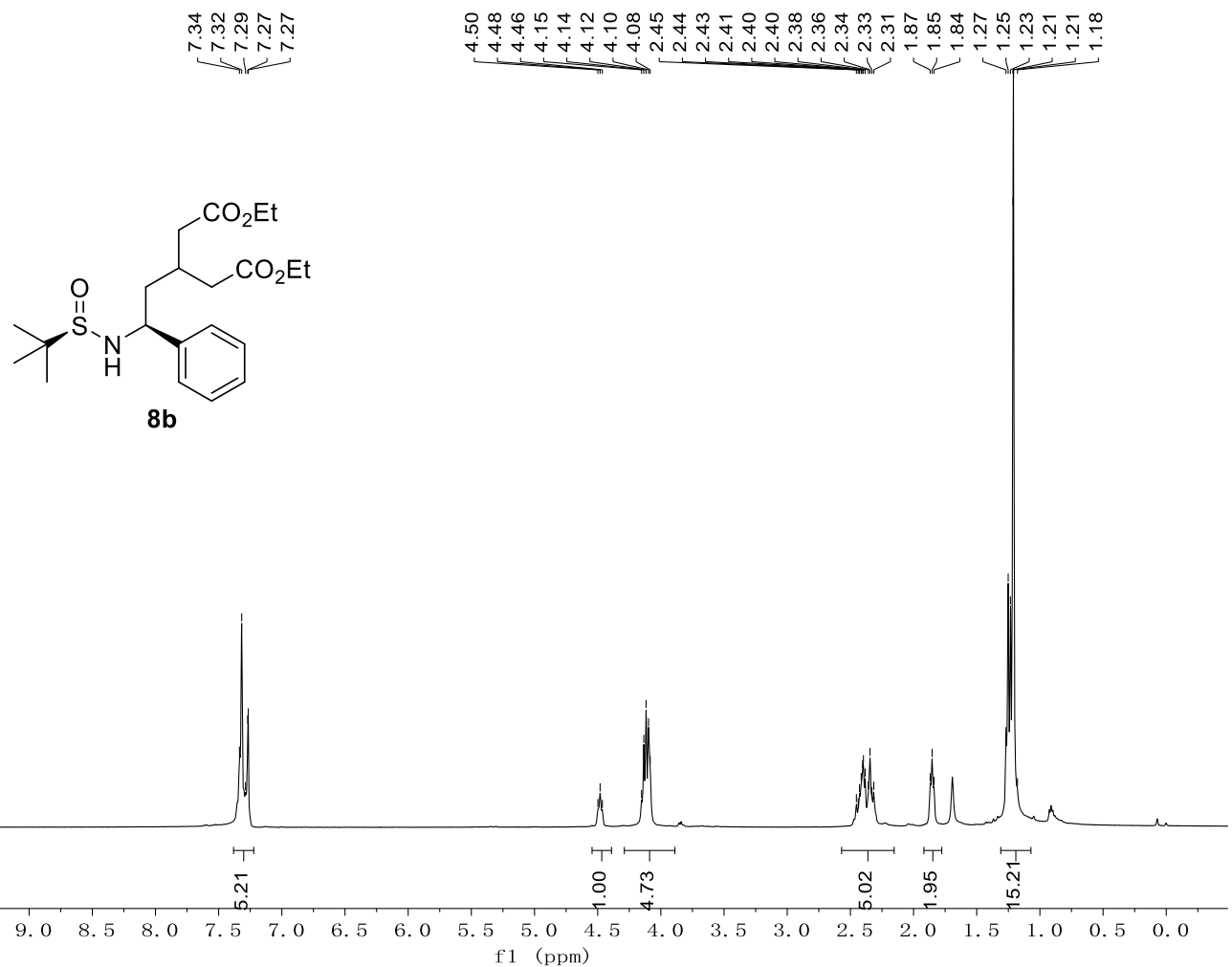

${ }^{13} \mathrm{C}\left\{{ }^{1} \mathrm{H}\right\}$ NMR of $\mathbf{8 b}\left(100 \mathrm{MHz}, \mathrm{CDCl}_{3}\right)$

焉

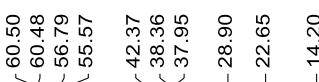<smiles>CCOC(=O)CC(CC(=O)OCC)CC(N[S@](=O)C(C)(C)C)c1ccccc1</smiles>

$8 b$

$\begin{array}{lllllllllllll}210 & 200 & 190 & 180 & 170 & 160 & 150 & 140 & 130 & 120 & 110 & 100 & 90\end{array}$ 
${ }^{1} \mathrm{H}$ NMR of $\mathbf{6 b}\left(400 \mathrm{MHz}, \mathrm{CDCl}_{3}\right)$

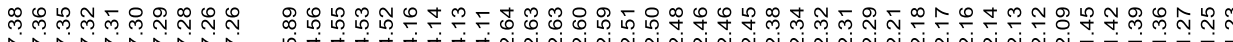

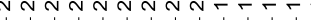

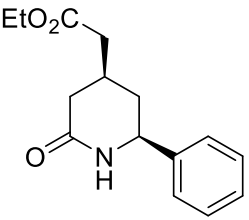

$6 b$

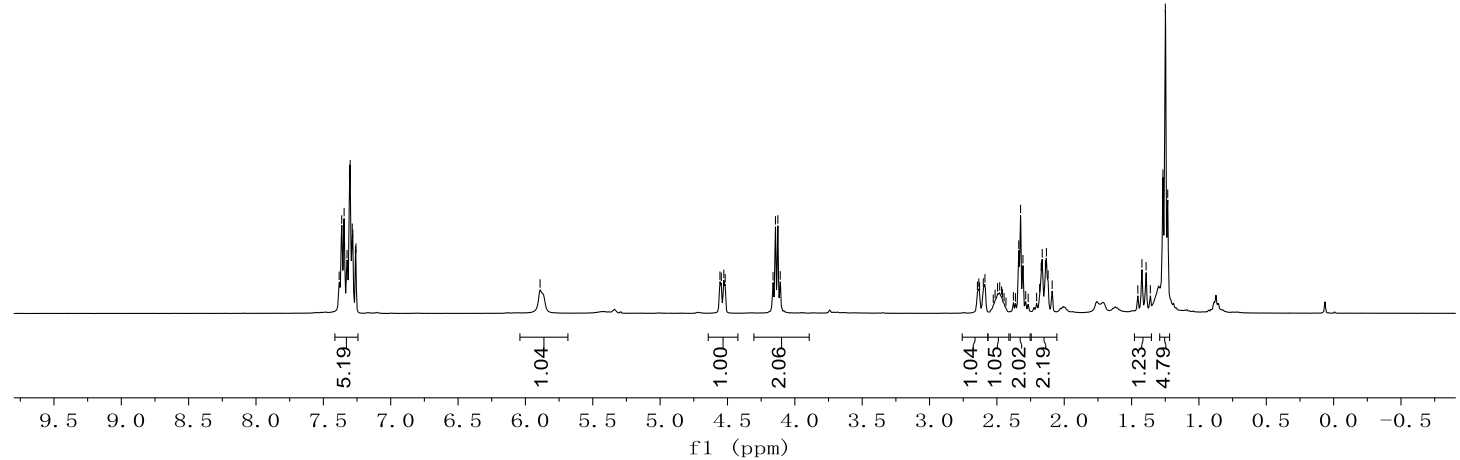

${ }^{13} \mathrm{C}\left\{{ }^{1} \mathrm{H}\right\} \mathrm{NMR}$ of $\mathbf{6 b}\left(100 \mathrm{MHz}, \mathrm{CDCl}_{3}\right)$

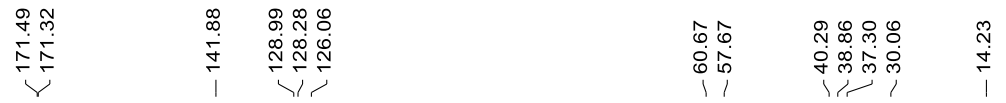<smiles>CCOC(=O)CC1CC(=O)NC(c2ccccc2)C1</smiles>

6b

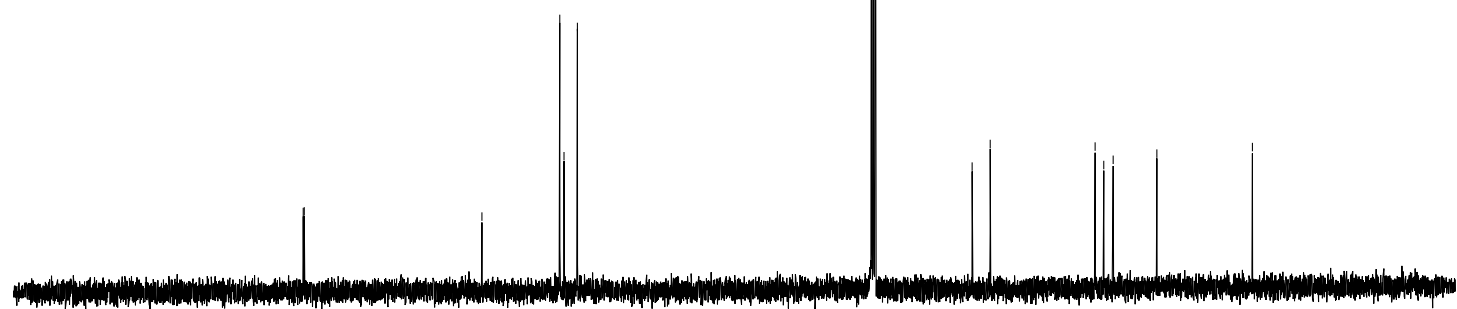

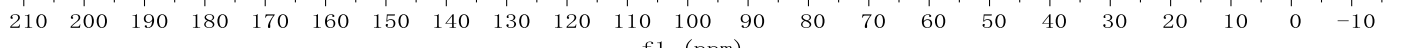
f1 (ppm) 
${ }^{1} \mathrm{H}$ NMR of $13 \mathbf{e}\left(400 \mathrm{MHz}, \mathrm{CDCl}_{3}\right)$

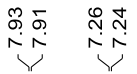

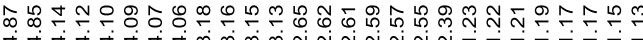

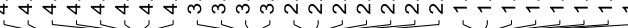

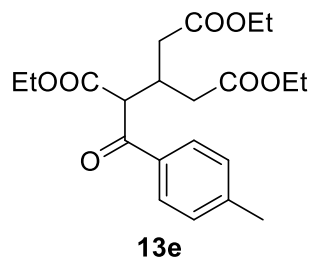

$13 e$
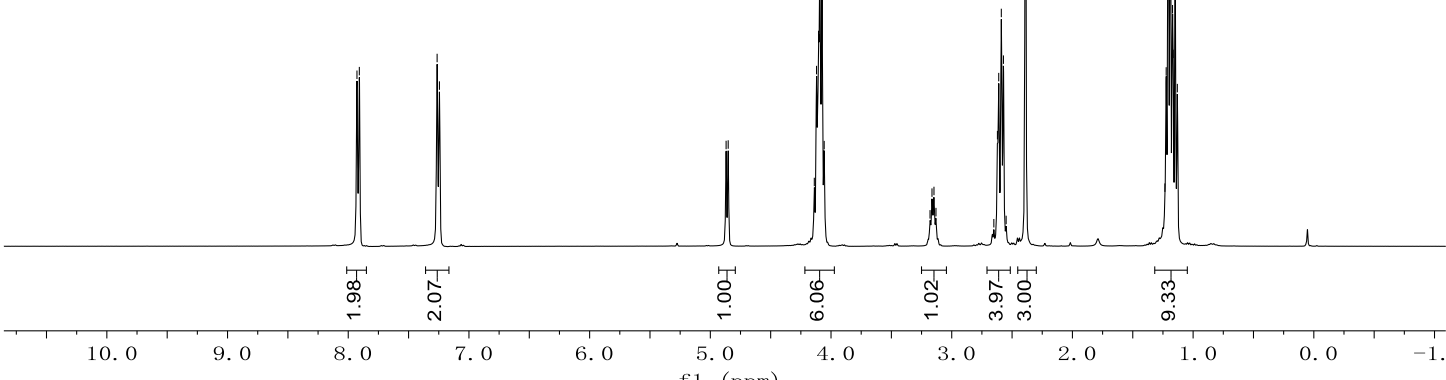

${ }^{13} \mathrm{C}\left\{{ }^{1} \mathrm{H}\right\}$ NMR of $13 \mathrm{e}\left(100 \mathrm{MHz}, \mathrm{CDCl}_{3}\right)$

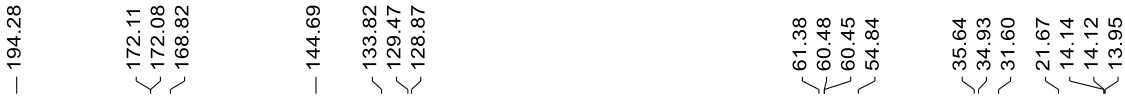

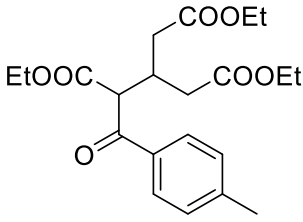

$13 e$

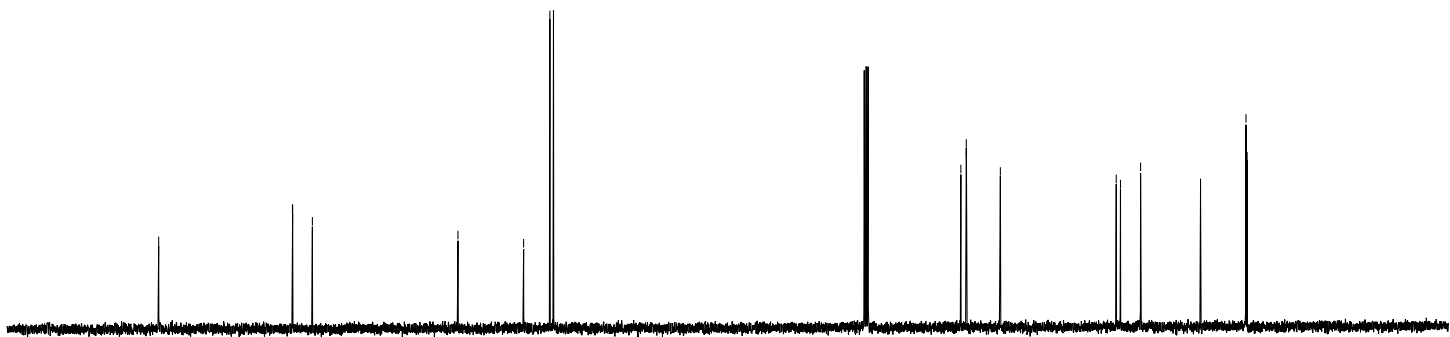

$\begin{array}{lllllllllllll}210 & 200 & 190 & 180 & 170 & 160 & 150 & 140 & 130 & 120 & 110 & 100 & 90\end{array}$ f1 (ppm) 
${ }^{1} \mathrm{H} \mathrm{NMR}$ of $4 \mathrm{e}\left(400 \mathrm{MHz}, \mathrm{CDCl}_{3}\right)$

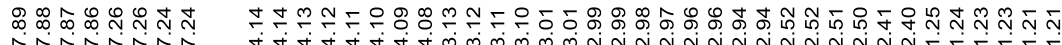

NNNNNN

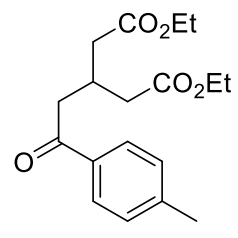

$4 e$

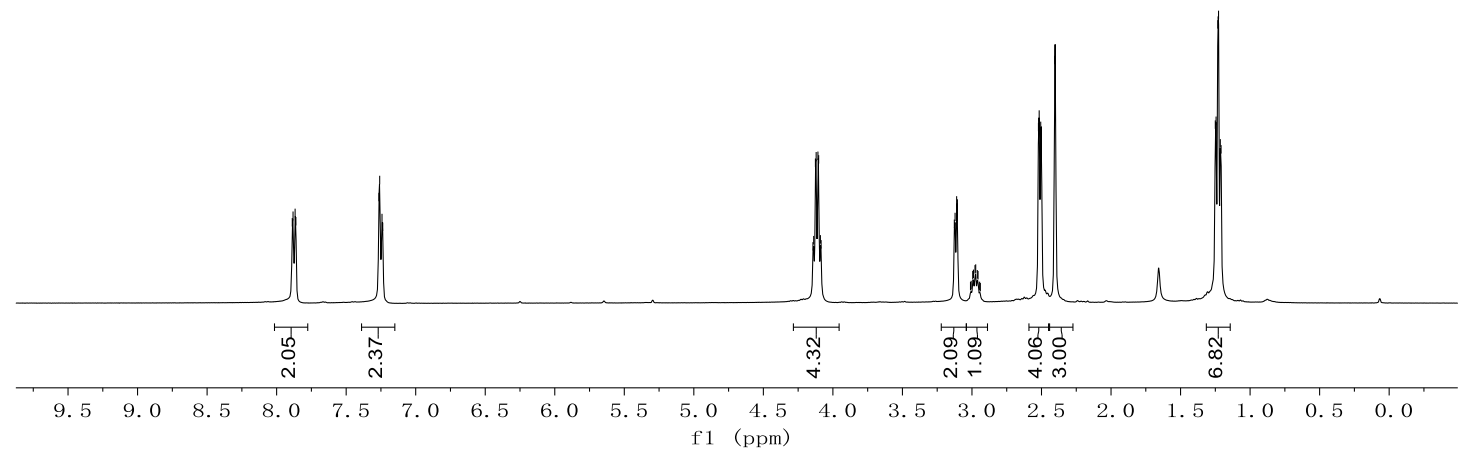

${ }^{13} \mathrm{C}\left\{{ }^{1} \mathrm{H}\right\}$ NMR of $4 \mathbf{e}\left(100 \mathrm{MHz}, \mathrm{CDCl}_{3}\right)$

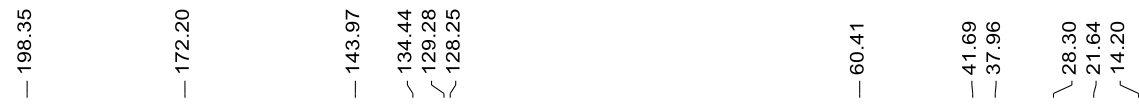<smiles>CCOC(=O)CC(CC(=O)OCC)CC(=O)c1ccc(C)cc1</smiles>

4 e

$\begin{array}{lllllllllllll}210 & 200 & 190 & 180 & 170 & 160 & 150 & 140 & 130 & 120 & 110 & 100 & 90\end{array}$ f1 (ppm) 
${ }^{1} \mathrm{H}$ NMR of $7 \mathbf{e}\left(400 \mathrm{MHz}, \mathrm{CDCl}_{3}\right)$

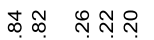

in

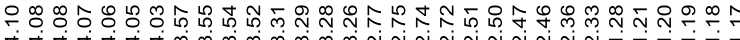

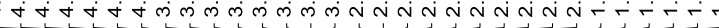

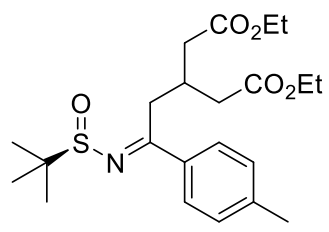

$7 e$

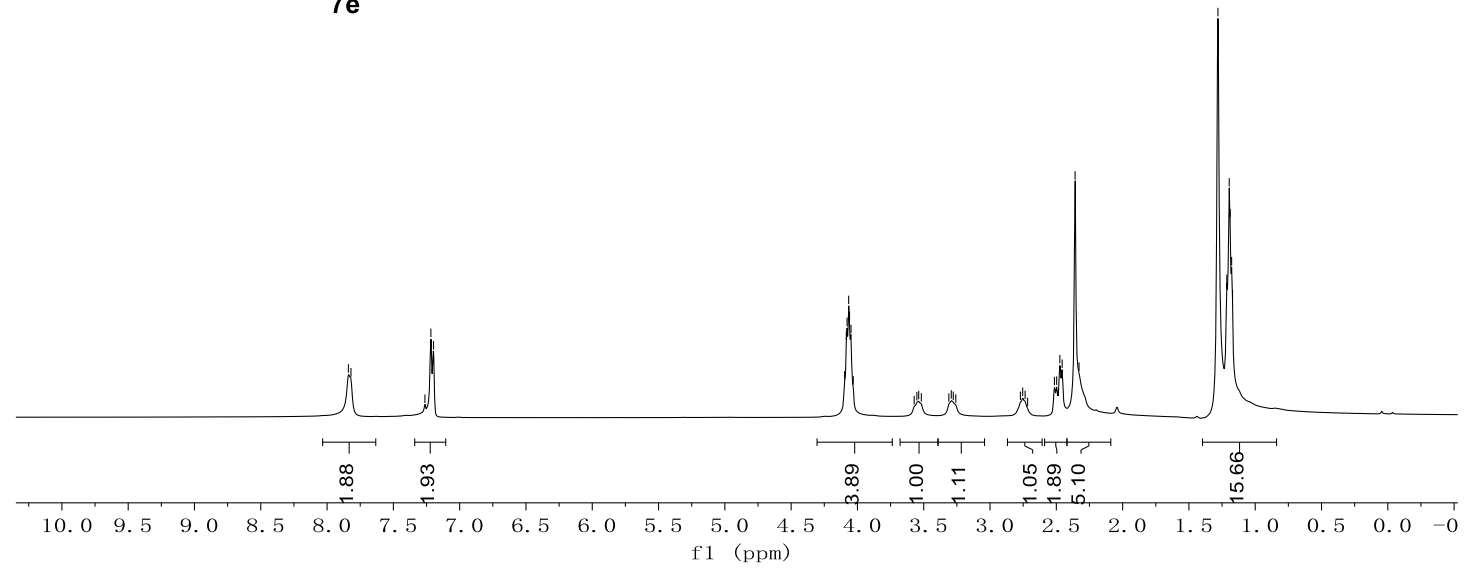

${ }^{13} \mathrm{C}\left\{{ }^{1} \mathrm{H}\right\} \mathrm{NMR}$ of $7 \mathrm{e}\left(100 \mathrm{MHz}, \mathrm{CDCl}_{3}\right)$

ma

ind

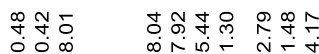

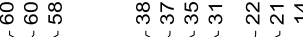<smiles>CCOC(=O)CC(CC(=O)OCC)CC(=NS(=O)C(C)(C)C)c1ccc(C)cc1</smiles>

$7 e$

$\begin{array}{lllllllllllll}210 & 200 & 190 & 180 & 170 & 160 & 150 & 140 & 130 & 120 & 110 & 100 & 90\end{array}$

f1 (ppm) 
${ }^{1} \mathrm{H}$ NMR of $8 \mathrm{e}\left(400 \mathrm{MHz}, \mathrm{CDCl}_{3}\right)$

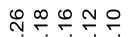

rinj

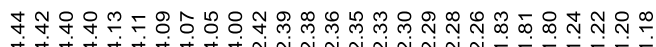

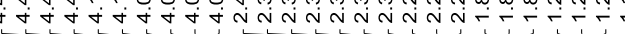<smiles>CCOC(=O)CC(CC(=O)OCC)CC(NS(=O)C(C)(C)C)c1ccc(C)cc1</smiles>

$8 e$

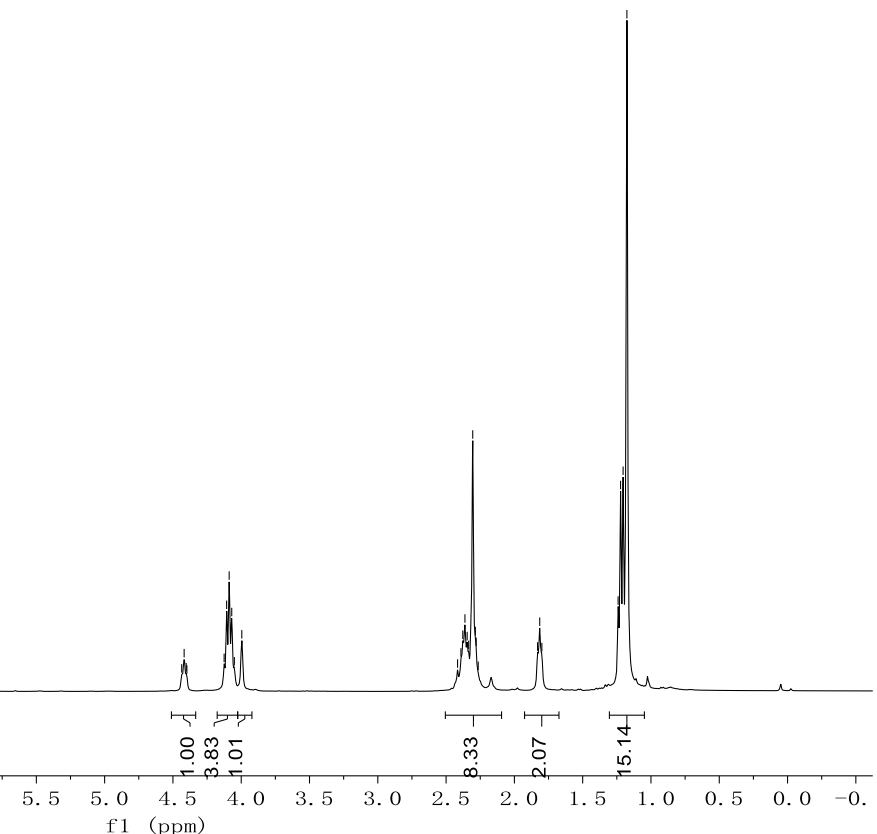

${ }^{13} \mathrm{C}\left\{{ }^{1} \mathrm{H}\right\}$ NMR of $8 \mathrm{e}\left(100 \mathrm{MHz}, \mathrm{CDCl}_{3}\right)$

品

$\stackrel{\infty}{\infty}$

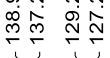

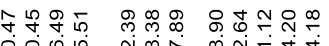

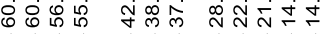<smiles>CCOC(=O)CC(CC(=O)OCC)CC(NS(=O)C(C)(C)C)c1ccc(C)cc1</smiles>

$8 e$

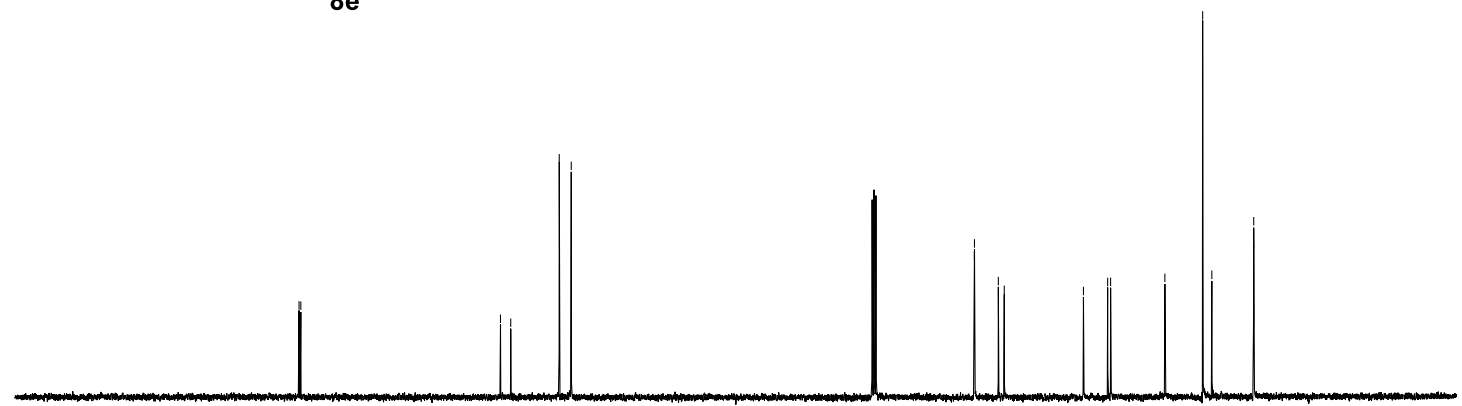

$\begin{array}{lllllllllllll}210 & 200 & 190 & 180 & 170 & 160 & 150 & 140 & 130 & 120 & 110 & 100 & 90\end{array}$ f1 (ppm) 
${ }^{1} \mathrm{H}$ NMR of $6 \mathbf{e}\left(400 \mathrm{MHz}, \mathrm{CDCl}_{3}\right)$

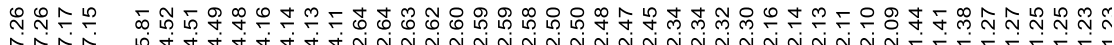

4
4

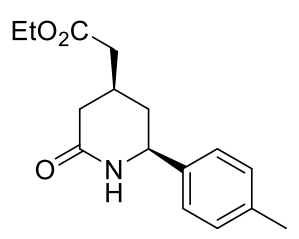

$6 e$

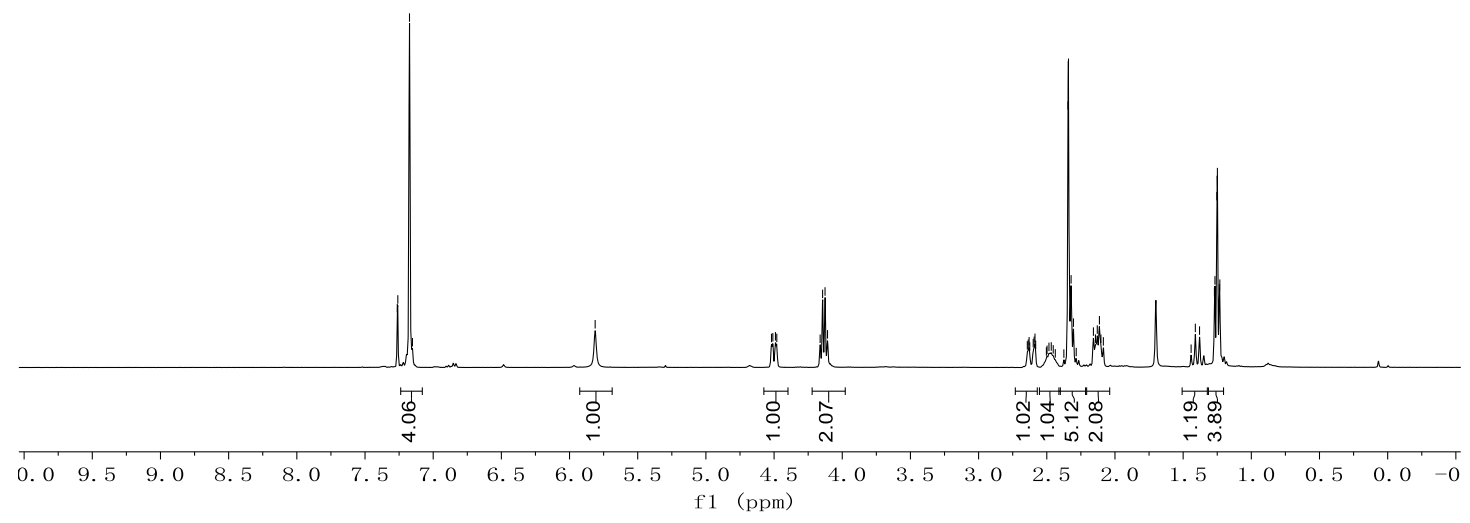

${ }^{13} \mathrm{C}\left\{{ }^{1} \mathrm{H}\right\} \mathrm{NMR}$ of $\mathbf{6 e}\left(100 \mathrm{MHz}, \mathrm{CDCl}_{3}\right)$

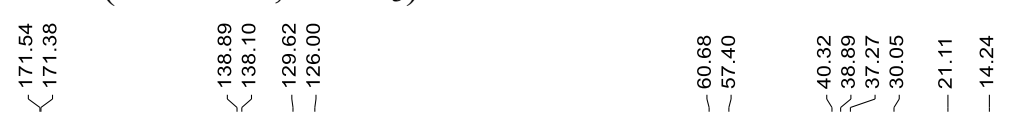<smiles>CCOC(=O)CC1CC(=O)N[C@H](c2ccc(C)cc2)C1</smiles>

6e

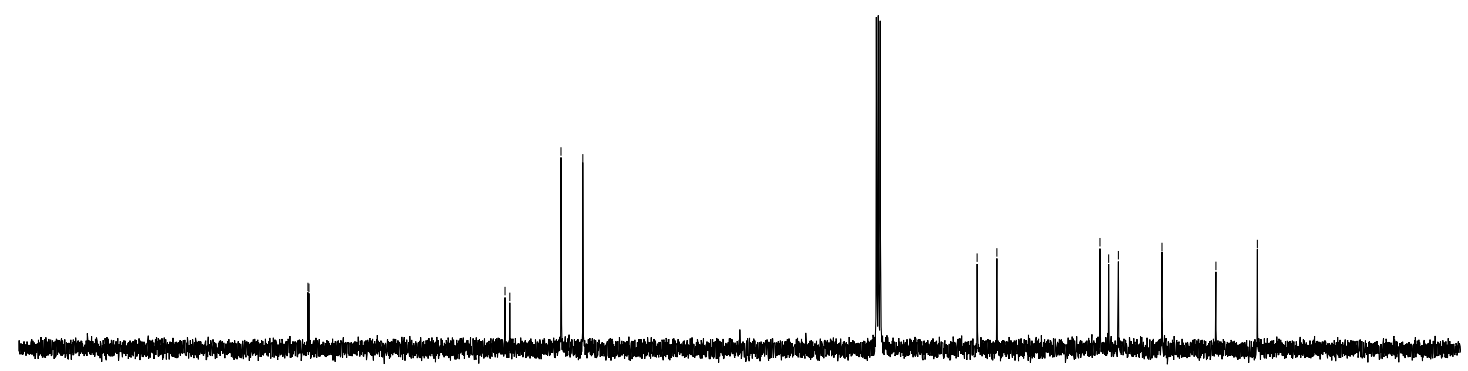

$\begin{array}{lllllllllllllllllllllllll}210 & 200 & 190 & 180 & 170 & 160 & 150 & 140 & 130 & 120 & 110 & 100 & 90 & 80 & 70 & 60 & 50 & 40 & 30 & 20 & 10 & 0 & -10\end{array}$ f1 (ppm) 
${ }^{1} \mathrm{H}$ NMR of GSM-1 (400 MHz, Methanol- $\left.d_{4}\right)$

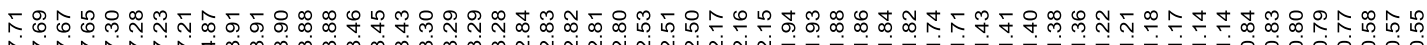

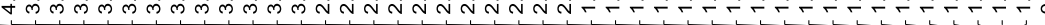

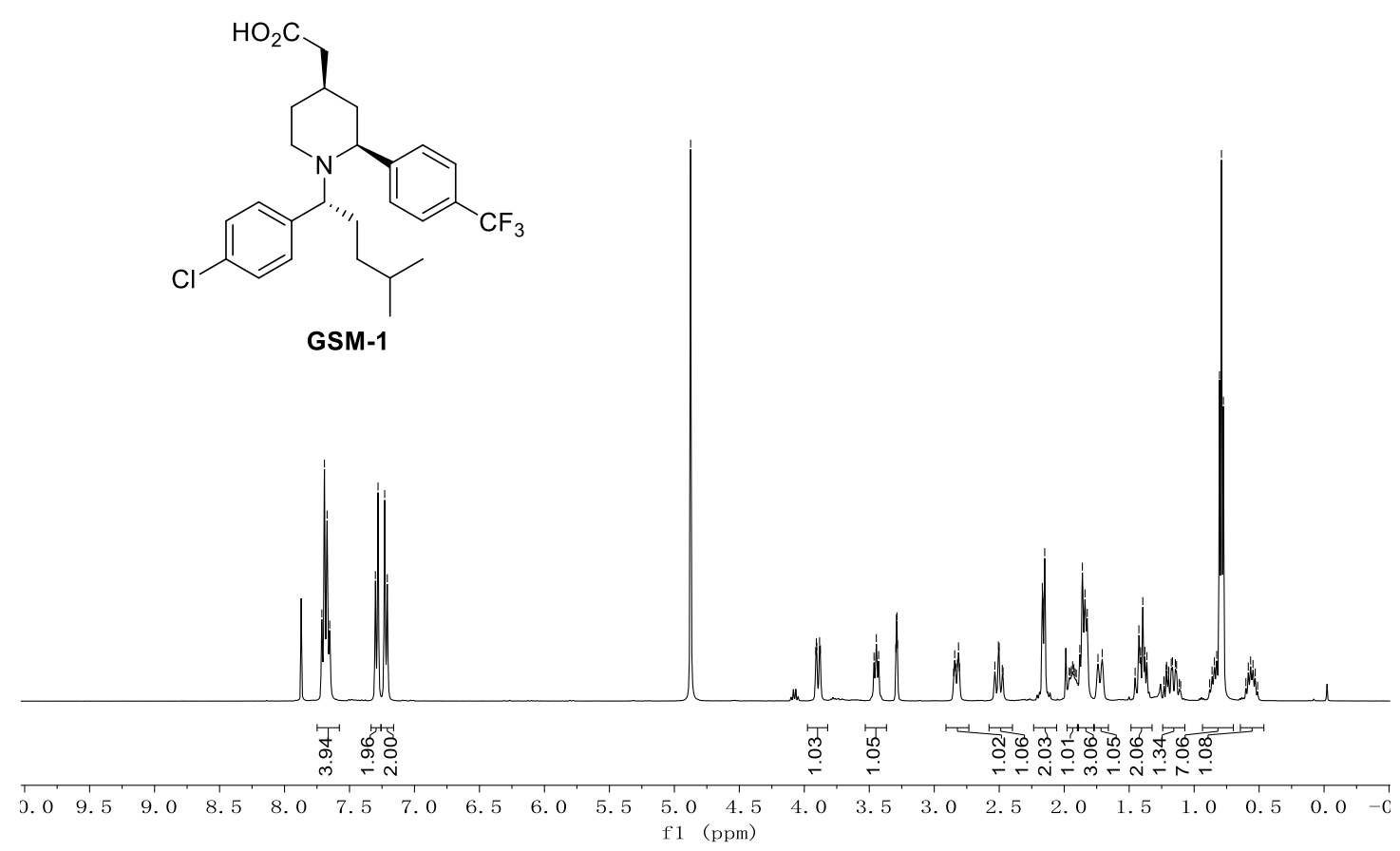

${ }^{13} \mathrm{C}\left\{{ }^{1} \mathrm{H}\right\}$ NMR of GSM-1 (100 MHz, Methanol- $\left.d_{4}\right)$

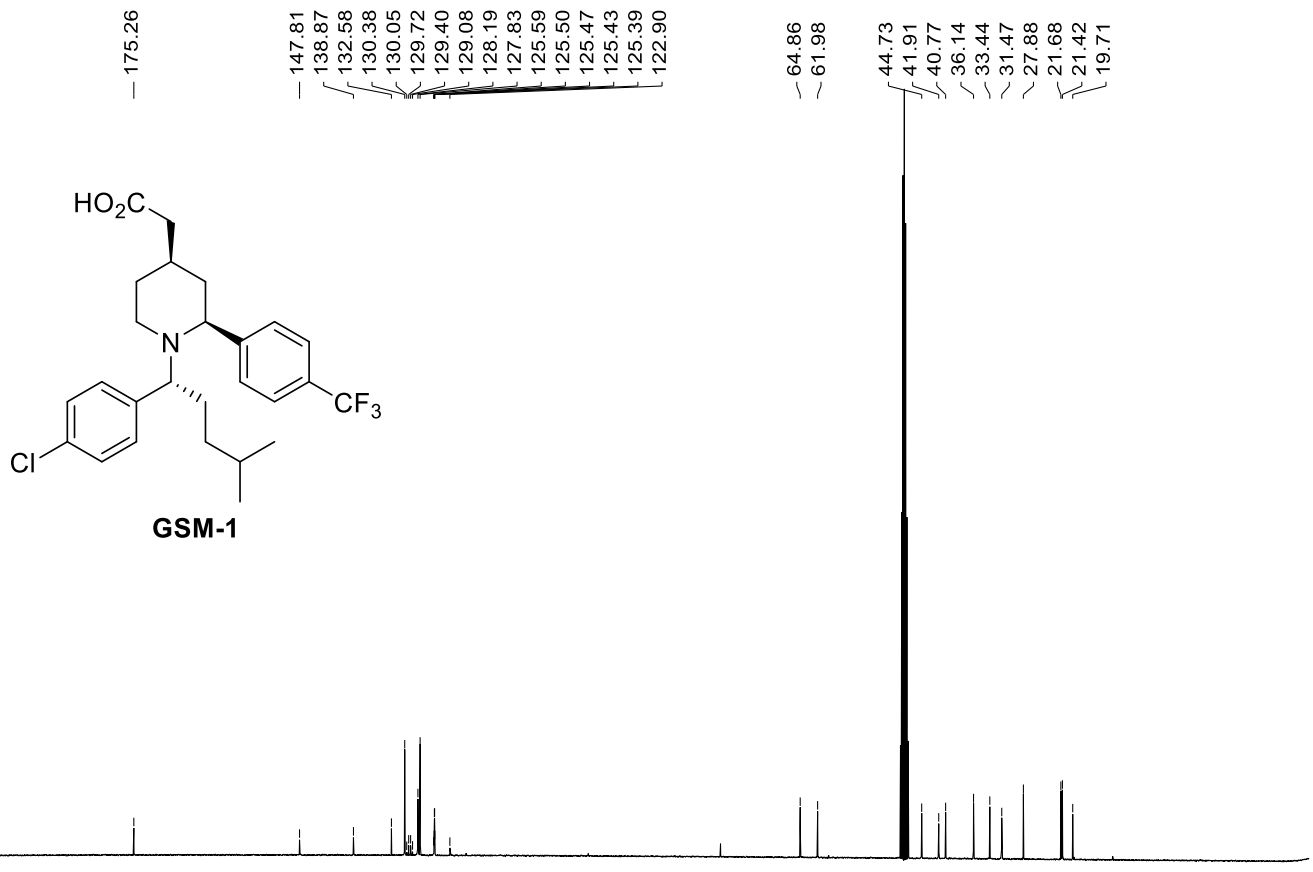

$\begin{array}{llllllllllllllllllllllllllllllll}210 & 200 & 190 & 180 & 170 & 160 & 150 & 140 & 130 & 120 & 110 & 100 & 90 & 80 & 70 & 60 & 50 & 40 & 30 & 20 & 10 & 0 & -10\end{array}$ f1 (ppm) 
${ }^{19}$ F NMR of GSM-1 (376 MHz, Methanol- $d_{4}$ )

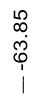

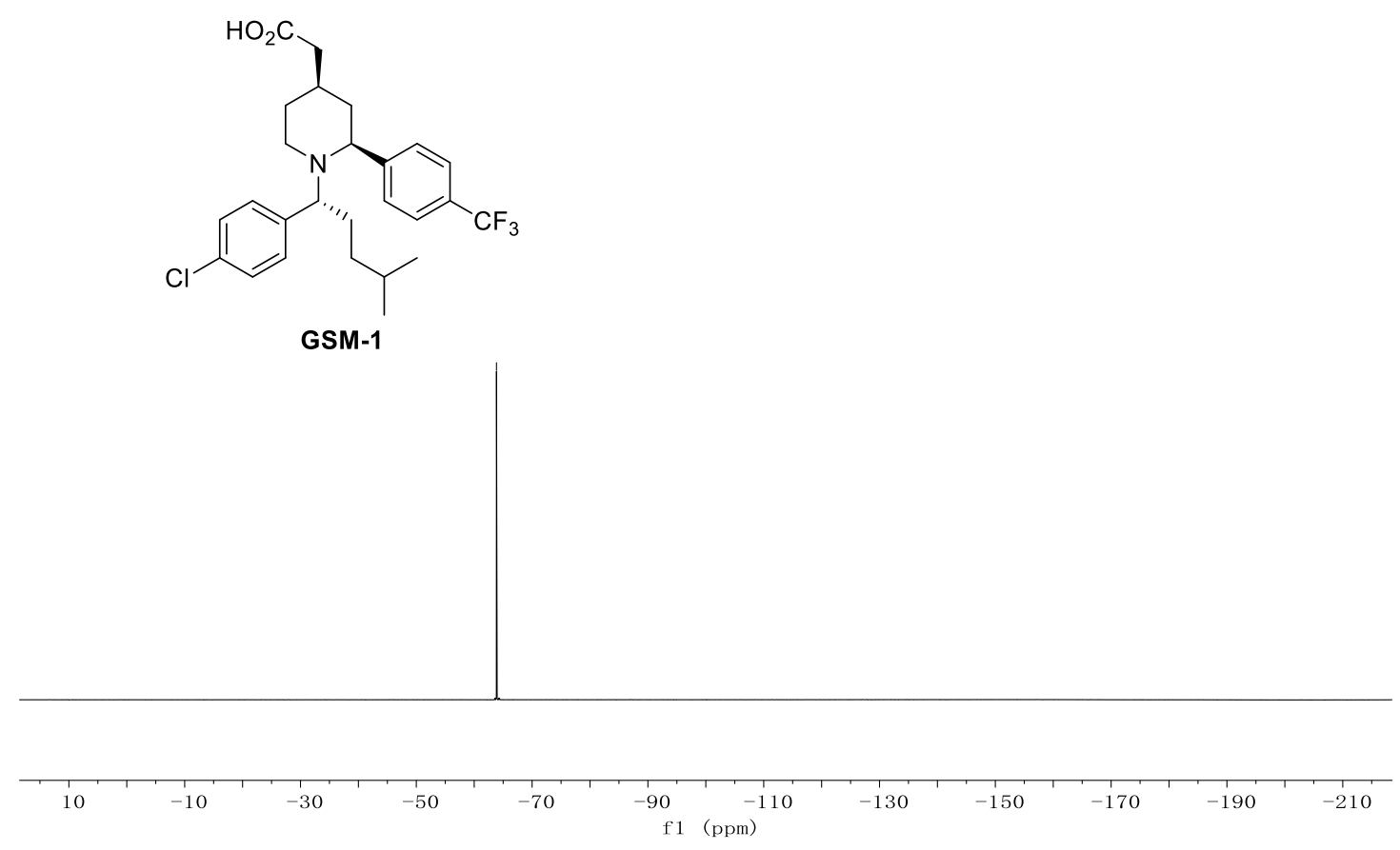


${ }^{1} \mathrm{H}$ NMR of 14 (400 MHz, Methanol- $\left.d_{4}\right)$

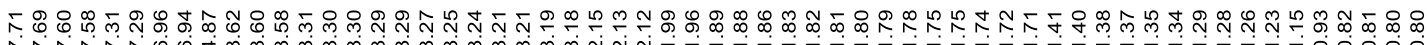

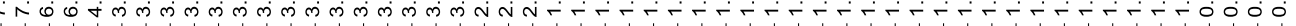

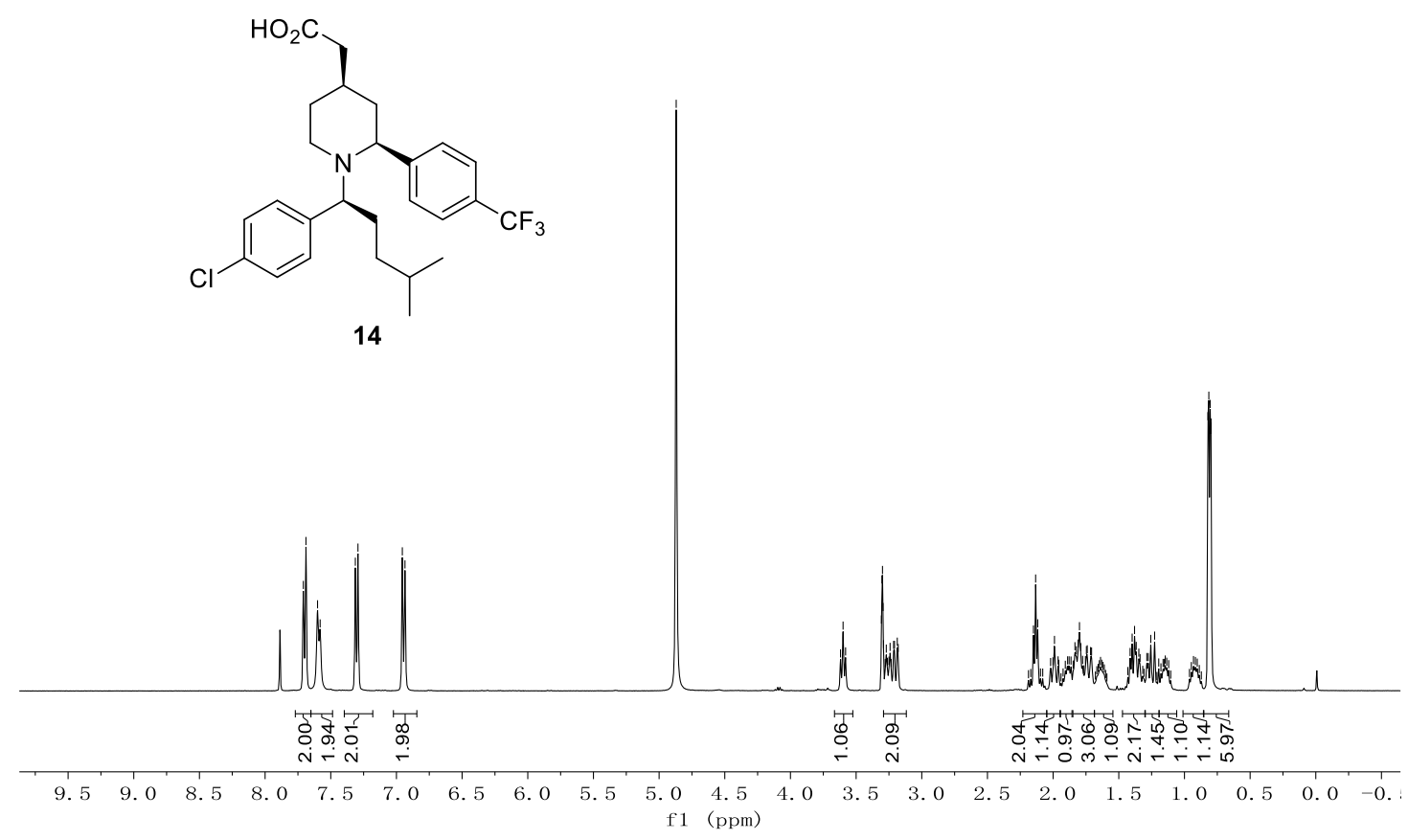

${ }^{13} \mathrm{C}\left\{{ }^{1} \mathrm{H}\right\}$ NMR of $14\left(100 \mathrm{MHz}\right.$, Methanol- $\left.d_{4}\right)$

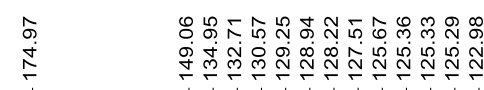<smiles>CC(C)CCC(c1ccc(Cl)cc1)N1CCC(CC(=O)O)CC1c1ccc(C(F)(F)F)cc1</smiles>

14

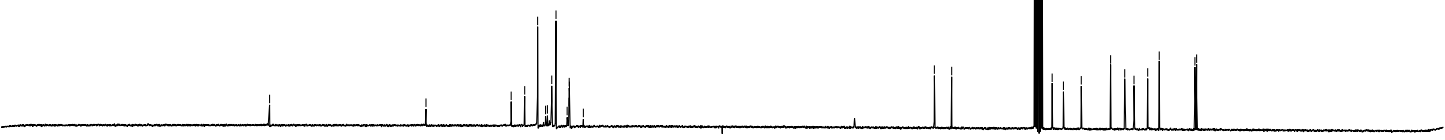

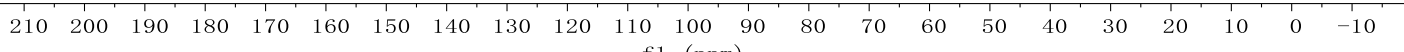
f1 (ppm) 
${ }^{19}$ F NMR of 14 (376 MHz, Methanol- $\left.d_{4}\right)$

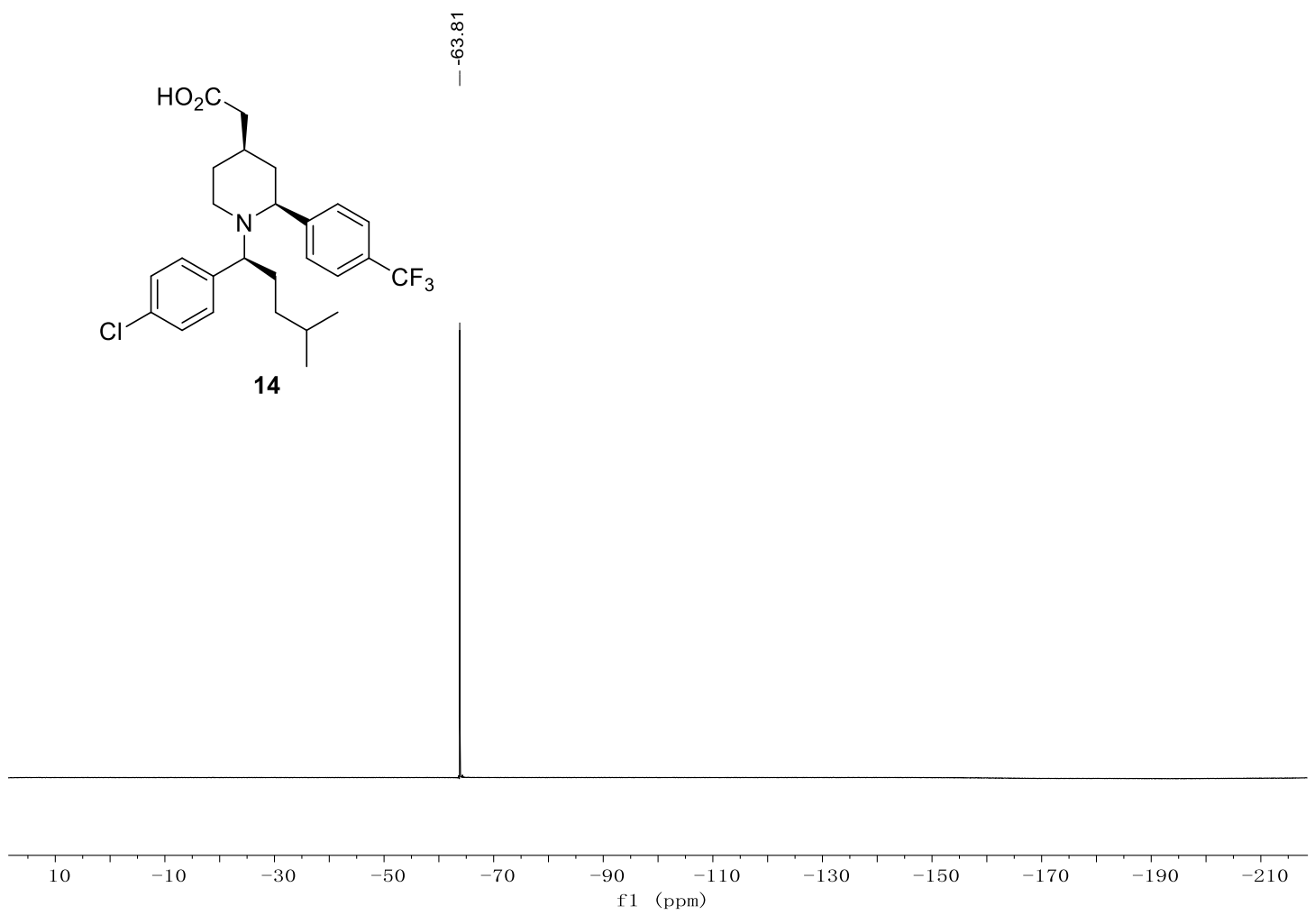

